\title{
Geometry of contact transformations and domains: orderability versus squeezing
}

\author{
YAKOV ELIASHBERG \\ SANG SEON KIM \\ LEONID POLTEROVICH
}

\begin{abstract}
Gromov's famous non-squeezing theorem (1985) states that the standard symplectic ball cannot be symplectically squeezed into any cylinder of smaller radius. Does there exist an analogue of this result in contact geometry? Our main finding is that the answer depends on the sizes of the domains in question: We establish contact nonsqueezing on large scales, and show that it disappears on small scales. The algebraic counterpart of the (non)-squeezing problem for contact domains is the question of existence of a natural partial order on the universal cover of the contactomorphisms group of a contact manifold. In contrast to our earlier beliefs, we show that the answer to this question is very sensitive to the topology of the manifold. For instance, we prove that the standard contact sphere is non-orderable while the real projective space is known to be orderable. Our methods include a new embedding technique in contact geometry as well as a generalized Floer homology theory which contains both cylindrical contact homology and Hamiltonian Floer homology. We discuss links to a number of miscellaneous topics such as topology of free loops spaces, quantum mechanics and semigroups.
\end{abstract}

53D10, 53D40; 53D35, 53D50

Dedicated to Dusa McDuff on the occasion of her $60^{\text {th }}$ birthday

\section{Introduction and main results}

\subsection{Contact (non)-squeezing}

Consider the standard symplectic vector space $\mathbb{R}^{2 n}$ endowed with the symplectic form $\omega=d p \wedge d q=\sum_{1}^{n} d p_{i} \wedge d q_{i}$. We often identify $\mathbb{R}^{2 n}$ with $\mathbb{C}^{n}$ and write $z=p+i q$ for the complex coordinate. Symplectic embeddings preserve the volume, and hence the Euclidean ball

$$
B^{2 n}\left(R_{1}\right):=\left\{\pi|z|^{2}<R_{1}\right\}
$$


cannot be symplectically embedded into $B^{2 n}\left(R_{2}\right)$ if $R_{2}<R_{1}$. Gromov's famous nonsqueezing theorem states that there are much more subtle obstructions for symplectic embeddings and, in particular, $B^{2 n}\left(R_{1}\right)$ cannot be symplectically embedded into the cylinder

$$
C^{2 n}\left(R_{2}\right):=B^{2}\left(R_{2}\right) \times \mathbb{R}^{2 n-2}
$$

when $R_{2}<R_{1}$, see [28]. This result led to the first non-trivial invariants of symplectic domains in dimension $2 n \geq 4$.

In the present paper we address the question whether there are any analogues of non-squeezing results in contact geometry. Consider the prequantization space of $\mathbb{R}^{2 n}$, that is the contact manifold $V=\mathbb{R}^{2 n} \times S^{1}, S^{1}=\mathbb{R} / \mathbb{Z}$, with contact structure $\xi=\operatorname{Ker}(d t-\alpha)$ where $\alpha$ is the Liouville form $\frac{1}{2}(p d q-q d p)$. Given a subset $D \subset \mathbb{R}^{2 n}$, write $\widehat{D}=D \times S^{1}$ for its prequantization. The naive attempt to extend the non-squeezing from $D$ to $\widehat{D}$ fails. It is is easy to show (see Proposition 1.24 and Section 2.2) that for any $R_{1}, R_{2}>0$ there exists a contact embedding of $\widehat{B}\left(R_{1}\right)$ into $\widehat{B}\left(R_{2}\right)$ which, for $n>1$, is isotopic to the inclusion through smooth embeddings into $V$. Furthermore, due to the conformal character of the contact structure, the domain $\widehat{B}(R)$ can be contactly embedded into an arbitrarily small neighborhood of a point in $V$ (see Corollary 1.25 below).

However, the situation becomes more sophisticated if one considers only those contact embeddings which come from globally defined compactly supported contactomorphisms of $(V, \xi)$. We write $\mathcal{G}=\operatorname{Cont}(V, \xi)$ for the group of all such contactomorphisms.

Given two open subsets $U_{1}$ and $U_{2}$ of a contact manifold $V$, we say that $U_{1}$ can be squeezed into $U_{2}$ if there exists a contact isotopy $\Psi_{t}$ : Closure $\left(U_{1}\right) \rightarrow V, t \in[0,1]$, such that $\Psi_{0}=\mathbf{1}$ and

$$
\Psi_{1}\left(\operatorname{Closure}\left(U_{1}\right)\right) \subset U_{2} .
$$

The isotopy $\left\{\Psi_{t}\right\}$ is called a contact squeezing of $U_{1}$ into $U_{2}$. If, in addition, $W \subset V$ is an open subset such that $\operatorname{Closure}\left(U_{2}\right) \subset W$ and $\Psi_{t}$ (Closure $\left.\left(U_{1}\right)\right) \subset W$ for all $t$, we say that $U_{1}$ can be squeezed into $U_{2}$ inside $W$. If the closure of $U_{1}$ is compact, the ambient isotopy theorem (see, for instance, Geiges [24]) guarantees that any squeezing of $U_{1}$ into $U_{2}$ inside $W$ extends to a contactomorphism from $\mathcal{G}$ whose support lies in $W .1$

\footnotetext{
${ }^{1}$ If the group $\mathcal{G}$ is not connected than the possibility to squeeze by an isotopy is stronger than by a global contactomorphism. All squeezing and non-squeezing results in this paper are proven in the strongest sense, ie, squeezing is always done by a contact isotopy while in our non-squeezing results we prove non-existence of the corresponding global contactomorphism.
} 
Remark 1.1 If a domain $U$ has a convex contact boundary then it admits a contact squeezing inside itself. Let us recall that a hypersurface $\Sigma$ in a contact manifold is called convex (see Eliashberg and Gromov [18]) if there exists a contact vector field $X$ which is transversal to $\Sigma$. Note that the vector field $-X$ is also contact, and hence one cannot assign to a convex hypersurface any canonical co-orientation. Giroux showed (see [26]) that in a 3-dimensional contact manifold any co-orientable surface can be made convex by a generic $C^{\infty}$-small perturbation. On the other hand, it is easy to check that the boundary of a domain $\widehat{D} \subset \mathbb{R}^{2 n} \times S^{1}$ is never convex.

Our main results concerning the contact squeezing problem are given in the next theorems.

Theorem 1.2 (Non-Squeezing) Assume that $R_{2} \leq m \leq R_{1}$ for some positive integer $m$. Then the closure of $\widehat{B}^{2 n}\left(R_{1}\right)$ cannot be mapped into $\widehat{C}^{2 n}\left(R_{2}\right)$ by a contactomorphism from $\mathcal{G}$. In particular, $\widehat{B}^{2 n}\left(R_{1}\right)$ cannot be squeezed into $\widehat{C}^{2 n}\left(R_{2}\right)$.

As a counterpoint to this result, we prove

Theorem 1.3 (Squeezing) Assume that $2 n \geq 4$. Then $\widehat{B}^{2 n}\left(R_{1}\right)$ can be squeezed into $\widehat{B}^{2 n}\left(R_{2}\right)$ for all $R_{1}, R_{2}<1$.

Remark 1.4 The restriction $n>1$ is essential: it was shown by Eliashberg in [16] that $\widehat{B}^{2}\left(R_{1}\right)$ cannot be squeezed into $\widehat{B}^{2}\left(R_{2}\right)$ for any $R_{1}>R_{2}$.

We do not know whether $\widehat{B}^{2 n}\left(R_{1}\right)$ can be squeezed into $\widehat{B}^{2 n}\left(R_{2}\right)$ or $\widehat{C}^{2 n}\left(R_{2}\right)$ when

$$
m+1>R_{1}>R_{2}>m
$$

for an integer $m \geq 1$.

Theorem 1.5 Assume that

$$
R_{2} \leq \frac{m}{k} \leq R_{1}<R_{3}<\frac{m}{k-1}
$$

for some integers $k, m \geq 1$. Then the closure of $\widehat{B}^{2 n}\left(R_{1}\right)$ cannot be mapped into $\widehat{B}^{2 n}\left(R_{2}\right)$ by any contactomorphism $\Phi \in \mathcal{G}$ with $\Phi\left(\widehat{B}^{2 n}\left(R_{3}\right)\right)=\widehat{B}^{2 n}\left(R_{3}\right)$. In particular, $\widehat{B}^{2 n}\left(R_{1}\right)$ cannot be squeezed into $\widehat{B}^{2 n}\left(R_{2}\right)$ inside $\widehat{B}^{2 n}\left(\frac{m}{k-1}\right)$.

In the case $m=1, k>1$ this result imposes a restriction on a squeezing of $\widehat{B}^{2 n}\left(\frac{1}{k}\right)$ into itself guaranteed by Theorem 1.3. Roughly speaking, such a squeezing requires some extra room. As we will see in Remark 1.23 below, this restriction is sharp: 
$\widehat{B}^{2 n}\left(\frac{1}{k}\right)$ can be squeezed into itself inside $\widehat{B}^{2 n}(\rho)$ for any $\rho>1 /(k-1)$.

The proofs of Theorems 1.2 and 1.5 are based on cylindrical contact homology theory (see Eliashberg, Givental and Hofer [17], Ustilovsky [44], Bourgeois [4] and Yau [48]) which is discussed in Sections 1.8 and 4 below. The (non)-squeezing phenomenon described above is closely related to the geometry of the group of contactomorphisms of the standard sphere $S^{2 n-1}$, see Sections 1.7 and 6.3 below. This resembles the link between symplectic non-squeezing and the geometry of the group of symplectomorphisms which was explored by Lalonde and McDuff in [34; 35].

\subsection{Negligible domains and symplectic capacities}

We say that a domain $X \subset V$ is negligible if every bounded open subset $U$ with Closure $(U) \subset X$ can be contactly squeezed into $\widehat{B}^{2 n}(r)$ for any $r>0$. We start this section with the following generalization of Theorem 1.3 above.

Theorem 1.6 The cylinder $\widehat{C}^{2 n}(1)$ is negligible when $2 n \geq 4$.

This result is sharp: indeed, for $R>1$ the cylinder $\widehat{C}^{2 n}(R)$ contains $\widehat{B}^{2 n}\left(R^{\prime}\right)$ with $R^{\prime}>1$ and hence it is not negligible due to Theorem 1.2.

The proof is given in Section 3.6 below. With this result at hand, we can present the transition from non-squeezing to squeezing in terms of symplectic capacities. For a bounded domain $U \subset \mathbb{R}^{2 n}$ define $\underline{c}(U)$ as the supremum of $R$ such that the ball $B^{2 n}(R)$ can be symplectically squeezed into $U$, and $\bar{c}(U)$ as the infimum of $R$ such that $U$ can be symplectically squeezed into the cylinder $C^{2 n}(R)$. These quantities are small modifications of the standard symplectic capacities. In particular, they are symplectic capacities in the symplectic category $\mathcal{O H}$ whose objects are open subsets of $\mathbb{R}^{2 n}$ and morphisms are symplectic embeddings induced by compactly supported Hamiltonian diffeomorphisms (see Cieliebak, Hofer, Latschev and Schlenk [11, Section 2.1] for the definition of a symplectic capacity in a symplectic category). Define the contact squeezing number $s q(U)$ as the infimum of $b \in \mathbb{R}^{+}$such that the domain $b^{-1 / 2} \cdot U \subset V$ is negligible. Let us emphasize that since $V$ is the prequantization space of $\mathbb{R}^{2 n}$, every compactly supported Hamiltonian isotopy of $\mathbb{R}^{2 n}$ lifts to a compactly supported contact isotopy of $V$. Therefore the contact squeezing number is invariant under compactly supported Hamiltonian diffeomorphisms. The next result is an immediate consequence of Theorems 1.2 and 1.6.

\section{Theorem 1.7}

$$
\underline{c}(U) \leq s q(U) \leq \bar{c}(U)
$$

for every bounded domain $U \subset \mathbb{R}^{2 n}$. 
As an immediate consequence of the theorem we get that the contact squeezing number is a symplectic capacity in category $\mathcal{O H}$.

\subsection{Preliminaries in contact geometry}

Let $(P, \eta)$ be a contact manifold with a co-oriented contact structure. Its symplectization $S P$ is defined as the set of all non-zero covectors from $T^{*} P$ whose kernel equals the contact hyperplane and which agree with its co-orientation. One checks that $S P$ is a symplectic submanifold of $T^{*} P$ if and only if $\eta$ is a contact structure. Thus it inherits from $T^{*} P$ the canonical Liouville 1-form $\alpha$ whose differential $\omega=d \alpha$ is the symplectic form. Note also that $S P$ is a (trivial) principal $\mathbb{R}_{+}$-bundle over $P$. The vector field $L$ generating the $\mathbb{R}_{+}-$action is called the Liouville field on $S P$. It satisfies $i_{L} \omega=\alpha$, and hence $\mathbb{R}_{+}$acts by conformally symplectic transformations. Any contact form for $\eta$ is a section of the bundle $S P \rightarrow P$. Its graph forms a hypersurface in $S P$ which is called a starshaped hypersurface.

Let $\beta$ be a contact form on $P$. Then using the $\mathbb{R}_{+}$-action one can identify $S P$ with $P \times \mathbb{R}_{+}$: the point $(x, \theta) \in S P$, where $x \in P$ and $\theta$ is a contact covector at $x$, corresponds to $(x, \theta / \beta) \in P \times \mathbb{R}_{+}$. After such an identification we write a point of $S P$ as $(x, u) \in P \times \mathbb{R}_{+}$and call $(x, u)$ the canonical coordinates on $S P$ associated to the contact form $\beta$. In canonical coordinates $\alpha=u \beta, \omega=d u \wedge \beta+u d \beta, L=\partial / \partial u$ and $\operatorname{graph}(\beta)=\{u=1\}$.

The following example is crucial for understanding what is going on below:

Example 1.8 Consider the standard contact sphere $\left(S^{2 n-1}, \eta\right)$ where the sphere $S^{2 n-1}$ is identified with $\partial B^{2 n}(1) \subset \mathbb{R}^{2 n}$ and $\eta=\operatorname{Ker}\left(\left.\alpha\right|_{S^{2 n-1}}\right)$. Its symplectization can be identified by an $\mathbb{R}_{+}$-equivariant symplectomorphism with $\left(\mathbb{R}^{2 n} \backslash\{0\}, d p \wedge d q\right)$, where the $\mathbb{R}_{+}$-action on $\mathbb{R}^{2 n} \backslash\{0\}$ is given by $z \rightarrow \sqrt{c} z$ for all $c \in \mathbb{R}_{+}$. Here every contact covector $(z, s \alpha) \in S P$, where $z \in P$ and $s>0$, corresponds to the point $\sqrt{s} z \in \mathbb{R}^{2 n} \backslash\{0\}$.

$\mathbb{R}_{+}$-equivariant Hamiltonian functions on $S P$ are called contact Hamiltonians. Every contactomorphism of $(P, \eta)$ uniquely lifts to an $\mathbb{R}_{+}$-equivariant symplectomorphism of $S P$. Moreover, there is a one-to-one correspondence between paths $\left\{f_{t}\right\}_{t \in[0,1]}$ of compactly supported contactomorphisms with $f_{0}=\mathbf{1}$ and contact Hamiltonian functions $F: S P \times[0 ; 1] \rightarrow \mathbb{R}$ which vanish outside $\tau^{-1}(K) \times[0 ; 1]$ where $K \subset P$ is a compact subset and $\tau: S P \rightarrow P$ the natural projection. We say the isotopy $\left\{f_{t}\right\}$ is generated by the contact Hamiltonian $F$.

Note that to every contact form $\beta$ on $P$ corresponds a unique contact Hamiltonian on $S P$ which equals 1 on $\operatorname{graph}(\beta)$. The projection of its Hamiltonian field to $P$ is a 
well defined vector field $R$ called the Reeb vector field of $\beta$. It is determined by the conditions $\beta(R)=1$ and $i_{R} d \beta=0$.

Let $Q \subset P$ be a hypersurface which is transversal to the contact structure. Every contact plane $\eta(x), x \in P$, carries a conformally canonical symplectic structure given by the differential of any contact form. The symplectic complement in $\eta(y), y \in Q$ to the hyperplane $T_{x} Q \cap \eta(x) \subset \eta(x)$ defines a field of lines $l(x) \subset T_{x} Q$. This field of lines integrates to a one-dimensional foliation which is called the contact characteristic foliation of $Q$.

\subsection{A partial order on contact transformations}

The results on contact (non)-squeezing presented in Section 1.1 above are closely related to the geometry of the group of contactomorphisms of the standard contact sphere $S^{2 n-1}$. In order to present these applications we need to recall some preliminaries from Eliashberg and Polterovich [19].

Let $(P, \eta)$ be a contact manifold. Denote by $\operatorname{Cont}_{0}(P, \eta)$ the identity component of the group of all compactly supported contactomorphisms of $(P, \eta)$. Let $\widetilde{C o n t}_{0}(P, \eta)$ be its universal cover. Given $\tilde{f}, \widetilde{g} \in \widetilde{\operatorname{Cont}}_{0}(P, \eta)$ we say that $\widetilde{f} \succeq \widetilde{g}$ if the element $\widetilde{f} \widetilde{g}^{-1}$ is represented by a path generated by a non-negative contact Hamiltonian. In other words, $\widetilde{f} \succeq \widetilde{g}$ if $\widetilde{f}$ can be reached from $\widetilde{g}$ by moving every point in the non-negative direction with respect to the contact structure $\eta$. Clearly, the relation $\succeq$ on $\overline{\operatorname{Cont}}_{0}(P, \eta)$ is reflexive and transitive. As shown in [19], for certain closed contact manifolds (eg, the unit cotangent bundle of the $n$-torus) it defines a genuine partial order. For the purpose of this discussion we shall call such manifolds orderable. The next proposition from [19] gives a useful necessary and sufficient condition for orderability of a closed contact manifold.

Proposition 1.9 Let $(P, \eta)$ be a closed contact manifold. The following conditions are equivalent:

(i) $(P, \eta)$ is non-orderable;

(ii) there exists a contractible loop $\phi: S^{1} \rightarrow \operatorname{Cont}_{0}(P, \eta)$ with $\phi(0)=\mathbf{1}$ which is generated by a strictly positive contact Hamiltonian.

It follows from Givental's theory of the non-linear Maslov index that the standard contact projective space $\mathbb{R} P^{2 n-1}$ is orderable (see [27;19]). In view of this, the authors of [19] tended to believe that its double cover $S^{2 n-1}$ is also orderable. Interestingly enough, this is not the case: 
Theorem 1.10 Assume that $2 n \geq 4$. There exists a positive contractible loop of contactomorphisms of the standard contact sphere $S^{2 n-1}$. In particular, the sphere is not orderable.

After the first version of this paper appeared in the arXiv, E Giroux informed us that Theorem 1.10 can be extracted from the existing literature as follows. The standard contact structure on $S^{2 n-1}$ coincides with the field of maximal complex subspaces tangent to the sphere, where we identify

$$
S^{2 n-1}=\partial B^{2 n} \subset \mathbb{C}^{n} .
$$

Thus the group of complex automorphisms of the unit ball $B^{2 n}$ acts by contact transformations on the boundary sphere. This group is isomorphic to $P U(n, 1)$. The cone of non-negative contact Hamiltonians on the sphere restricts to a tangent cone $C$ in the Lie algebra $p u(n, 1)$, which is invariant under the adjoint representation. It turns out that 25 years ago G Olshanskii [39] completely characterized those cones which give rise to a genuine partial order on the universal cover of the group. Applying Olshanskii's criterion, one gets that $C$ does not generates a genuine partial order. Thus, a fortiori $S^{2 n-1}$ is non-orderable. For reader's convenience, we present more details on the Oshanskii criterion and illustrate its application to non-orderability of $S^{3}$ in Appendix B. In the paper, we chose another route and use a method which enables us to extend Theorem 1.10 to more general contact manifolds (cf Remark 8.3 in Appendix B). This extension is presented in Section 1.6 below (see Theorem 1.16 whose proof occupies Section 3).

Existence of positive contractible loops is a manifestation of "symplectic flexibility". However, such loops themselves exhibit a rigid behavior. We illustrate this in the case of the standard contact sphere $S^{2 n-1}$ with $n \geq 2$. Let

$$
\Delta=\left\{f_{t, s}\right\}, t \in S^{1}, s \in[0,1],
$$

be a homotopy of a positive contractible loop $\left\{f_{t, 1}\right\}$ to the constant loop $\left\{f_{t, 0}\right\} \equiv \mathbf{1}$. Assume that $f_{0, s}=\mathbf{1}$ for all $s$. Write $F_{s}, s \in[0,1]$, for the contact Hamiltonian on $\left(\mathbb{R}^{2 n} \backslash\{0\}\right) \times S^{1}$ generating the loop $\left\{f_{t, s}\right\}, t \in S^{1}$. Put

$$
\mu(\Delta):=-\min _{z, s, t} \frac{F_{S}(z, t)}{\pi|z|^{2}} .
$$

Theorem 1.11 Assume that $2 n \geq 4$. Then

(i) $\mu(\Delta) \geq 1$ for every homotopy $\Delta$ of a positive contractible loop of contactomorphisms of the sphere $S^{2 n-1}$ to the constant loop; 
(ii) moreover, this estimate is sharp: $\inf _{\Delta} \mu(\Delta)=1$.

We shall see in Section 1.7 that the inequality $\mu(\Delta) \geq 1$ follows from Non-Squeezing Theorem 1.2.

\subsection{Liouville manifolds}

In this section we introduce the class of Liouville-fillable contact manifolds which are in the focus of our study. Let $(M, \omega)$ be a connected symplectic manifold which satisfies the following conditions:

(i) There exists a complete vector field $L$ on $M$ such that $\omega=d \alpha$ with $\alpha=i_{L} \omega$. This implies that the flow $L^{t}, t \in \mathbb{R}$, of $L$ acts on $M$ by conformally symplectic diffeomorphisms.

(ii) There exists a closed connected hypersurface $P \subset M$ which is transversal to $L$ and bounds an open domain $U \subset M$ with compact closure such that $M=U \sqcup \bigcup_{t \geq 0} L^{t} P$.

The vector field $L$ is called a Liouville field, and a symplectic manifold $(M, \omega)$ with a fixed Liouville field $L$ (or, which is the same, with a fixed primitive $\alpha=i_{L} \omega$ which is called a Liouville form) is called a Liouville manifold. We will call starshaped any hypersurface $P$ and any domain $U$ in the Liouville manifold $(M, \omega, L)$ which satisfy the condition (ii). Given any starshaped domain $U \subset M$ its repeller $\operatorname{Core}_{P}(M)=$ $\bigcap_{t \in \mathbb{R}^{+}} L^{-t}(U)$ is called the core of the Liouville manifold $(M, \omega, L)$.

Put $\beta:=\left.\alpha\right|_{P}$. The transversality condition in (ii) is equivalent to the requirement that $\eta:=\operatorname{Ker} \beta$ is a contact structure on $P$. Moreover, the symplectization $S P$ can be naturally symplectically identified with the set $M_{*, P}=\bigcup_{t \in \mathbb{R}} L^{t}(P)$ as follows: Consider the splitting $S P=P \times(0, \infty)$ associated to the form $\beta$ (see Section 1.3 above) and identify a point $(x, u) \in S P$ with $L^{\log u} x \in M$. Under this identification the form $\alpha$ and the vector field $L$ on $M$ correspond to the Liouville form and Liouville vector field on $S P$ respectively. Note that in coordinates $(x, u)$ we have $\alpha=u \beta$.

We claim that the decomposition $M=M_{*, P} \sqcup \operatorname{Core}_{P}(M)$ does not depend on the choice of a starshaped hypersurface $P$. Indeed, let $Q$ be another starshaped hypersurface. Consider the subset $X=M_{*, P} \cap M_{*, Q}$, and denote by $Y$ its projection to $P$ along the trajectories of $L$. Note that $X$ is non-empty: otherwise we have $M_{*, Q} \subset U$ which is absurd since $M_{*, Q}$ has infinite volume and $U$ has compact closure by condition (ii) above. Hence $Y$ is an open non-empty subset of $P$. Furthermore, since $M \backslash U=\bigcup_{t \geq 0} L^{t} P$, every trajectory of $L$ starting on $P$ leaves any compact subset $K \supset U$ in finite time. The same holds true for $Q$. Therefore, all segments of 
trajectories of $L$ with endpoints on $P \cup Q$ have uniformly bounded length (understood as the length of the corresponding time-interval). This readily yields that $Y$ is closed, and hence $Y=P$, since $P$ is connected. Hence, $Q$ is a starshaped hypersurface in $M_{*, P}=S P$, and the claim follows. In particular, the core of $M$ is independent of $P$. We write $\operatorname{Core}(M)$ for $\operatorname{Core}_{P}(M)$, and $M_{*}$ for $M \backslash \operatorname{Core}(M)$.

Furthermore, the projection $\pi_{P, Q}$ of $Q$ to $P$ along the trajectories of $L$ establishes a contactomorphism between the contact structures $\operatorname{Ker}\left(\left.\alpha\right|_{T P}\right)$ and $\operatorname{Ker}\left(\left.\alpha\right|_{T Q}\right)$. Thus we associated to a Liouville manifold $(M, \omega, L)$ a canonical contact manifold, defined as a family of contact manifolds $\left\{\left(P,\left.\operatorname{Ker} \alpha\right|_{T P}\right)\right\}$ and contactomorphisms $\pi_{P, Q}: Q \rightarrow P$ satisfying $\pi_{P, Q} \circ \pi_{Q, R}=\pi_{P, R}$ where $P, Q, R$ run over the set of all starshaped hypersurfaces in $M$. This contact manifold admits a more geometric description. Note that $\mathbb{R}_{+}$acts freely on $M \backslash \operatorname{Core}(M)$ by the formula $c * x=L^{\log c} x$ and we have $\left(L^{\log c}\right)^{*} \alpha=c \alpha$. Hence the plane field $\{\alpha=0\}$ on $M_{*}$ is invariant under the $\mathbb{R}_{+}$-action. It descends to a contact plane field $\eta_{\infty}$ on $P_{\infty}:=M_{*} / \mathbb{R}_{+}$. We will call $\left(P_{\infty}, \eta_{\infty}\right)$ the ideal contact boundary of the Liouville manifold, and in the sequel will use both of its descriptions. Let us emphasize that the symplectization of $P_{\infty}$ is canonically identified with $M_{*}$, and hence we have a canonical decomposition

$$
M=S P_{\infty} \sqcup \operatorname{Core}(M) .
$$

Contact manifolds $\left(P_{\infty}, \eta_{\infty}\right)$ arising in this way are called Liouville-fillable, and we refer to $(M, \omega, L)$ as a Liouville filling of $\left(P_{\infty}, \eta_{\infty}\right)$.

Example 1.12 The standard symplectic linear space $\left(\mathbb{R}^{2 n}, d p \wedge d q\right)$ equipped with the vector field $L=\frac{1}{2}\left(p \frac{\partial}{\partial p}+q \frac{\partial}{\partial q}\right)$ is a Liouville manifold. The Liouville form $\alpha$ equals $\frac{1}{2}(p d q-q d p)$. It follows from Example 1.8 above that the ideal contact boundary of $\mathbb{R}^{2 n}$ is the standard contact sphere $S^{2 n-1}$, and the core equals $\{0\}$. Our convention is that the zero-dimensional space (that is, the point) is a Liouville symplectic manifold: it coincides with its core, and its ideal contact boundary is empty.

Example 1.13 The cotangent bundle $T^{*} X$ of a closed manifold $X$ equipped with the standard symplectic form $d p \wedge d q$ and the Liouville vector field $p \frac{\partial}{\partial p}$ is a Liouville manifold. Its ideal contact boundary is called the space of co-oriented contact elements of $X$ and is denoted $\mathbb{P}_{+} T^{*} X$. The core coincides with the zero section.

Example 1.14 (Weinstein manifolds) A Liouville manifold $(M, \omega, L)$ is called Weinstein if the vector field $L$ is gradient-like for an exhausting (that is, proper and bounded from below) Morse function $h$ on $M$. Contact manifolds $(P, \eta)$ arising as ideal contact boundaries of Weinstein manifolds are called Weinstein-fillable [18]. This 
class of contact manifolds has an alternative description in terms of complex geometry, see [15]: namely, they appear as strictly pseudo-convex boundaries of Stein domains equipped with the field of complex tangent subspaces of maximal dimension. (Recall that a Stein domain is a sublevel set of an exhausting plurisubharmonic function on a Stein manifold.) All critical points of the function $h$ as above have Morse index $\leq n=\frac{1}{2} \operatorname{dim} W$. If all the indices do not exceed $n-k$ for $1 \leq k \leq n$, the Weinstein manifold $M$ is called $k$-subcritical. Otherwise, it is called critical. For instance, $\mathbb{R}^{2 n}$ is $n$-subcritical (and hence, of course, $k$-subcritical for every $k \in[1, n]$ ), while $\mathbb{P}_{+} T^{*} X$ is critical. A Weinstein-fillable contact manifold is called $k$-subcritical if it admits a $k$-subcritical Weinstein filling, and critical otherwise.

Example 1.15 (Stabilization) Let $(M, \omega, L),\left(M^{\prime}, \omega^{\prime}, L^{\prime}\right)$ be two Liouville manifolds. Their product $\left(M \times M^{\prime}, \omega \oplus \omega^{\prime}, L \oplus L^{\prime}\right)$ is again a Liouville manifold. In the case when

$$
\left(M^{\prime}, \omega^{\prime}, L^{\prime}\right)=\left(\mathbb{R}^{2 n}, d p \wedge d q, \frac{1}{2}\left(p \frac{\partial}{\partial p}+q \frac{\partial}{\partial q}\right)\right)
$$

the obtained Liouville manifold is called the $n$-stabilization of $(M, \omega, L)$. The significance of this notion is due to the following result by $\mathrm{K}$ Cieliebak [8]: Every $k$-subcritical Weinstein manifold is a $k$-stabilization of another Weinstein manifold.

\subsection{On orderability of Liouville-fillable manifolds}

The next theorem is a generalization of Theorem 1.10:

Theorem 1.16 For any Liouville manifold $(M, \omega, L)$ the ideal contact boundary of its $n$-stabilization is not orderable provided that $n \geq 2$.

The proof is given in Section 3 below. Theorem 1.10 corresponds to the case when $M$ is a point. Our discussion in Example 1.15 above yields the following corollary.

Corollary 1.17 Weinstein-fillable 2-subcritical contact manifolds are not orderable.

It is interesting to confront this result with the following

Theorem 1.18 Let $X$ be a closed manifold. Assume that either $\pi_{1}(X)$ is finite, or $\pi_{1}(X)$ has infinitely many conjugacy classes. Then the space $\mathbb{P}_{+} T^{*} X$ of co-oriented contact elements of $X$ with its canonical contact structure is orderable. 
This result generalizes Theorem 1.3.B. from [19]. We refer to Section 5.3 for the proof. Interestingly enough, it is unknown whether Theorem 1.18 covers all closed manifolds. This depends on the answer to the following long-standing open question in group theory due to J Makowsky [36]: does there exist an infinite finitely-presented group with finitely many conjugacy classes? (See Baumslag, Myasnikov and Shpilrain [2] for further discussion.) Note that spaces of co-oriented contact elements, or unit cotangent bundles, are examples of contact manifolds with critical Weinstein filling. We refer the reader to Section 6 for further discussion on fillability and orderability.

\subsection{Orderability versus squeezing}

Let $(M, \omega, L)$ be a Liouville manifold with the ideal contact boundary $(P, \eta)$. Write $\alpha$ for the Liouville $1-$ form on $M$. Consider the prequantization space of $M$ which is defined as the contact manifold $\left(V=M \times S^{1}, \xi=\operatorname{Ker}(\alpha-d t)\right)$. In this section we present a basic link between contact (non)-squeezing in $V$ and the orderability of $P$.

Convention In what follows we identify $S P$ with $M_{*}=M \backslash \operatorname{Core}(M)$ and extend all contact Hamiltonians from $M_{*}$ to $M$ by setting them equal to 0 on $\operatorname{Core}(M)$. Of course, this extension is not, necessarily, smooth along $\operatorname{Core}(M)$.

An open domain of $V$ with smooth compact boundary is called fiberwise starshaped if it intersects the fibers $M \times\{t\}, t \in S^{1}$ along starshaped domains and its boundary is transversal to the fibers.

Suppose that $P$ is non-orderable, that is it admits a positive contractible loop of contactomorphisms. Denote by $\left\{f_{t}\right\}$ the corresponding loop of $\mathbb{R}_{+}-$equivariant symplectomorphisms of $M_{*}$. Let

$$
\left\{f_{t, s}\right\}, t \in S^{1}, s \in[0,1],
$$

be a homotopy of $\left\{f_{t, 1}\right\}=\left\{f_{t}\right\}$ to the constant loop $\left\{f_{t, 0}\right\} \equiv \mathbf{1}$. Assume that $f_{0, s}=\mathbf{1}$ for all $s$. Write $F_{s}, s \in[0,1]$, for the contact Hamiltonian on $V$ generating the loop $\left\{f_{t, s}\right\}, t \in S^{1}$. Let $E: M \rightarrow \mathbb{R}$ be a positive contact Hamiltonian. For each $R>0$ let us consider a domain

$$
A(R)=\{E<R\} \times S^{1} \subset V .
$$

Roughly speaking, the next theorem shows that every homotopy of a positive contractible loop of contactomorphisms of $P$ to the constant loop serves as a "squeezing tool" for the domain $A(R)$ provided that $R$ is small enough. 
Theorem 1.19 Suppose there exists $\mu>0$ such that

$$
F_{s}(z, t)>-\mu E(z)
$$

for all $z \in M_{*}, t \in S^{1}, s \in[0 ; 1]$. Then

(i) there exists $\gamma>0$ such that $A(R)$ can be contactly squeezed into $A\left(\frac{R}{1+\gamma R}\right)$ for all $R<\mu^{-1}$

(ii) $A(R)$ can be contactly squeezed into itself inside $A(\rho)$ for all

$$
R<\mu^{-1} \text { and } \rho>\frac{1}{R^{-1}-\mu} .
$$

Moreover the squeezing in (i) and (ii) can be performed in the class of fiberwise starshaped domains.

The proof is based on an elementary geometric construction from [19] which links together positive paths of contactomorphisms of $P$ and fiberwise starshaped domains in $V$. Let $\phi=\left\{f_{t}\right\}, t \in[0 ; 1], f_{0}=\mathbf{1}$, be a path of contactomorphisms of $P$ generated by a positive time-periodic contact Hamiltonian $F: V \rightarrow \mathbb{R}$. Define a domain

$$
U(\phi):=\{F<1\} \subset V .
$$

Vice versa, every fiberwise starshaped domain of $V$ is of the form $\{F<1\}$ for a unique positive time-periodic contact Hamiltonian $F$, and thus corresponds to the positive path of contactomorphisms of $P$ generated by $F$.

Example 1.20 (Standard rotation) The rotation $e_{t}(z)=e^{2 \pi i t} z, t \in \mathbb{R}$, defines a positive path of contact transformations of $P=S^{2 n-1}$ generated by the contact Hamiltonian $\pi|z|^{2}$. Fix $R>0$ and consider the path $\epsilon:=\left\{e_{t / R}\right\}$. Clearly, the corresponding starshaped domain $U(\epsilon)$ is simply

$$
\widehat{B}^{2 n}(R)=B^{2 n}(R) \times S^{1} \subset \mathbb{C}^{n} .
$$

A crucial feature of the correspondence $\phi \rightarrow U(\phi)$ is as follows:

Lemma 1.21 Let $\left\{\phi_{s}\right\}, s \in[0 ; 1]$ be a homotopy of paths of contactomorphisms of $P$ through positive paths with fixed end points. Then there exists an ambient contact isotopy $\Psi_{s}: V \rightarrow V$ with $\Psi_{0}=\mathbf{1}$ and $\Psi_{s}\left(U\left(\phi_{0}\right)\right)=U\left(\phi_{s}\right)$ for all $s \in[0 ; 1]$.

The proof virtually repeats the one of Lemma 3.1.B in [19]. 
Remark 1.22 Note, however, that existence of a global contact isotopy which deforms $U\left(\phi_{0}\right)$ into $U\left(\phi_{1}\right)$ through starshaped domains implies only that there exists a homotopy of positive paths of contactomorphisms

$$
\phi_{s}=\left\{f_{s}^{t}\right\}_{t \in[0,1]}, s \in[0,1],
$$

such that $f_{s}^{0}=\mathbf{1}$ and $f_{s}^{1}=g_{s}^{-1} \circ f_{0}^{1} \circ g_{s}$ for a certain path of contactomorphisms $g_{s}: P \rightarrow P, s \in[0,1]$.

Sketch of the proof of Theorem 1.19 (ii) Denote by $e_{t}$ the Hamiltonian flow of $E$ on $M_{*}$. Denote by $\epsilon$ the path of contactomorphisms of $P$ corresponding to $e_{t / R}, t \in[0 ; 1]$. With this notation $A(R)=U(\epsilon)$.

Consider the following homotopy of the path $\epsilon:=\left\{e_{t / R}\right\}$ with fixed endpoints:

$$
\epsilon_{s}=\left\{e_{t / R} f_{t, s}\right\}, t \in S^{1}, s \in[0 ; 1] .
$$

The Hamiltonian $H_{s}$ generating $\epsilon_{s}$ is given by

$$
H_{s}(z, t)=R^{-1} E(z)+F_{S}\left(e_{-t / R^{z}}, t\right) .
$$

The assumptions of the theorem guarantee that

$$
H_{S}(z, t)>R^{-1} E(z)-\mu E\left(e_{-t / R} z\right) .
$$

The energy conservation law for $\left\{e_{t}\right\}$ yields $E\left(e_{-t / R} z\right)=E(z)$, and therefore

$$
H_{S}(z, t)>\left(R^{-1}-\mu\right) E(z)>\rho^{-1} E(z) \text {. }
$$

Hence, by Lemma 1.21 the family of closed domains Closure $\left(U\left(\epsilon_{S}\right)\right)$ provides a contact isotopy of Closure $\left(U\left(\epsilon_{0}\right)\right)=\operatorname{Closure}(A(R))$ inside $A(\rho)$. Recall that we started with a positive loop, hence $F_{1}>0$. Therefore $H_{1}>R^{-1} E(z)$ which yields Closure $\left(U\left(\epsilon_{1}\right)\right) \subset U\left(\epsilon_{0}\right)=A(R)$, and thus we get the desired squeezing of $A(R)$ into itself inside $A(\rho)$.

The argument above contains a gap: Hamiltonians $H_{S}(z, t)$ are in general not 1-periodic in time, and hence formally speaking domains $U\left(\epsilon_{s}\right)$ are not well defined. This can be corrected by an appropriate "smoothing of corners" argument: see Section 2 below for the complete proof of Theorem 1.19.

Theorem 1.19 applied to the case when $M=\mathbb{R}^{2 n}=\mathbb{C}^{n}$ and $E(z)=\pi|z|^{2}$ enables us to reduce Theorem 1.11(i) to Theorem 1.2 and Theorem 1.3 to Theorem 1.11(ii). Note that in this case $A(R)=B^{2 n}(R)$. 
Theorem 1.2 $\Rightarrow$ Theorem 1.11(i) Assume on the contrary that $\mu(\Delta)<1$. Then Theorem 1.19(i) gives us a squeezing of Closure $(\widehat{B}(1))$ into its interior, in contradiction to Theorem 1.2.

Theorem 1.11(ii) $\Rightarrow$ Theorem 1.3 Assume that $0<R_{2}<R_{1}<1$. Choose a homotopy $\Delta=\left\{f_{t, s}\right\}$ of a positive contractible loop $\left\{f_{t, 1}\right\}$ of contactomorphisms of the sphere to the constant loop $\left\{f_{t, 0}\right\} \equiv \mathbf{1}$ with $\mu(\Delta)<1 / R_{1}$. Theorem 1.19(i) guarantees that for every $R \leq R_{1}$, the domain $\widehat{B}^{2 n}(R)$ can be squeezed into $\widehat{B}^{2 n}(v(R))$ where the function $v(R)$ is given by

$$
v(R)=\frac{R}{1+\gamma R}
$$

for some $\gamma>0$. Note that $0<v(R)<R$ for $R>0$, and the $N$-th iteration $v^{(N)}(R)=$ $(v \circ \ldots \circ v)(R)$ satisfies $v^{(N)}(R) \rightarrow 0$ as $N \rightarrow+\infty$. Choose $N$ large enough so that $v^{(N)}\left(R_{1}\right)<R_{2}$. Hence, iterating our construction $N$ times we get a squeezing of $\widehat{B}^{2 n}\left(R_{1}\right)$ into $\widehat{B}^{2 n}\left(R_{2}\right)$. This completes the proof.

Remark 1.23 The second part of Theorem 1.11 states that for every $\varepsilon>0$ there exists a homotopy $\Delta$ of a positive contractible loop to the constant loop with $\mu(\Delta) \leq 1+\varepsilon$. Hence, Theorem 1.5 provides a sharp restriction on any squeezing of $\widehat{B}^{2 n}\left(\frac{1}{k}\right)$ into itself, where $k>1$ is an integer. Indeed, apply Theorem 1.19 (ii) with $R=\frac{1}{k}$ and $\mu(\Delta) \leq 1+\varepsilon$. We get a squeezing of $\widehat{B}^{2 n}\left(\frac{1}{k}\right)$ into itself inside $\widehat{B}^{2 n}(\rho)$ provided $\rho>1 /(k-\mu)$. Taking $\varepsilon$ small enough we see that $\rho$ can be chosen arbitrarily close to $\frac{1}{k-1}$.

Let us mention also that there is an alternative way to use a positive (not necessarily contractible) loop of contactomorphisms of the sphere $S^{2 n-1}$ for producing contact embeddings of domains in $\mathbb{R}^{2 n} \times S^{1}$, see Section 2.2 below. A special (trivial) case of this construction enables us to prove the following result which we already have mentioned in Section 1.1 above.

Proposition 1.24 For all $R_{1}, R_{2}>0$ there exists a contact embedding of $\widehat{B}^{2 n}\left(R_{1}\right)$ into $\widehat{B}^{2 n}\left(R_{2}\right)$. For $n>1$ this embedding can be chosen isotopic through smooth embeddings to the natural inclusion

$$
\widehat{B}^{2 n}\left(R_{1}\right) \hookrightarrow \mathbb{R}^{2 n} \times S^{1} .
$$

Of course, in general this isotopy cannot be made contact. Furthermore, assume that $R_{2} \leq m<R_{1}$ for some $m \in \mathbb{N}$. Then the embedding guaranteed by the proposition does not extend to a compactly supported contactomorphism of $V$ in view of the Non-Squeezing Theorem above. 
Proof For any integer $N>0$ define a map $F_{N}: \mathbb{R}^{2 n} \times S^{1} \rightarrow \mathbb{R}^{2 n} \times S^{1}$ by the formula

$$
(z, t) \mapsto\left(v(z) e^{2 \pi N i t} z, t\right),
$$

where

$$
v(z)=\frac{1}{\sqrt{1+N \pi \sum_{1}^{n}\left|z_{j}\right|^{2}}} .
$$

It is straightforward to check that $F_{N}$ is a contactomorphism of $\mathbb{R}^{2 n} \times S^{1}$. It maps $\widehat{B}^{2 n}\left(R_{1}\right)$ onto $\widehat{B}^{2 n}(R)$ with $R=\frac{R_{1}}{1+N R_{1}} \underset{N \rightarrow \infty}{\rightarrow} 0$. For $n>1$ and an even $N$ the map $F_{N}$ is isotopic to the inclusion.

The following fact is well known to specialists. However, we provide it with a proof for a reader's convenience.

Corollary 1.25 For every $R>0$ there exists a contact embedding of $\widehat{B}(R)$ into an arbitrarily small neighbourhood of a point in any contact manifold.

Proof By the contact Darboux theorem it suffices to show that for every $R>0, \epsilon>0$ there exists a contact embedding of $\widehat{B}(R)$ into Euclidean ball of radius $\epsilon$ in the standard contact space

$$
\left(\mathbb{R}^{2 n+1}, \operatorname{Ker}\left(d z+\frac{1}{2}(p d q-q d p)\right) .\right.
$$

Consider the Euclidean circle of radius $\epsilon / 2$ with the center at 0 lying in the $\left(p_{1}, q_{1}\right)-$ plane. This circle is transversal to the contact structure, and hence by the relative Darboux theorem (eg see [24], Example 2.33) its sufficiently small neighbourhood is contactomorphic to $\widehat{B}(r)$ with some $r>0$. But $\widehat{B}(R)$ can be contactly embedded into $\widehat{B}(r)$ in view of Proposition 1.24. This completes the proof.

\subsection{Contact homology}

The proof of Non-Squeezing Theorem 1.2 is based on contact homology theory. Here we present a brief outline of contact homology adjusted to our purposes and refer the reader to Section 4 below for more details and generalizations. ${ }^{2}$ Let $(M, \omega, L)$ be a Liouville manifold whose first Chern class $c_{1}(T M)$ vanishes on $\pi_{2}(M)$. Put

\footnotetext{
${ }^{2}$ The more general contact homology groups constructed in Section 4 have a more sophisticated grading which involves the set of free homotopy classes of loops in $M$. The simpler version presented in this section corresponds to the component associated to the class of contractible loops.
} 
$V=M \times S^{1}$ and set $\lambda=d t-\alpha$, where $\alpha$ is a Liouville form on $M$. Equip $V$ with the contact structure $\xi=\operatorname{Ker}(\lambda)$.

Consider the set $\mathcal{U}$ consisting of all fiberwise starshaped open domains in $V$ and their images under the group $\mathcal{G}=\operatorname{Cont}(V, \xi)$ of compactly supported contactomorphisms of $(V, \xi)$. Contact homology $\mathrm{CH}(U)$ of a domain $U \in \mathcal{U}$ is a $\mathbb{Z}$-graded vector space over $\mathbb{Z}_{2}$. Every inclusion $U_{1} \subset U_{2}$ gives rise to a morphism

$$
\text { in: } \mathrm{CH}\left(U_{1}\right) \rightarrow \mathrm{CH}\left(U_{2}\right) \text {. }
$$

Every contactomorphism $\Phi \in \mathcal{G}$ induces an isomorphism

$$
\Phi_{\sharp}: \mathrm{CH}(U) \rightarrow \mathrm{CH}(\Phi(U)) .
$$

These morphisms preserve the grading and have the following properties (the diagrams below are commutative):

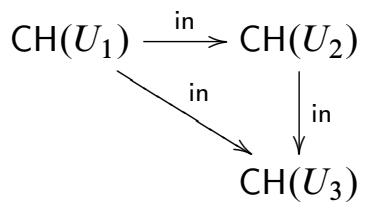

provided $U_{1} \subset U_{2} \subset U_{3}$ are domains from $\mathcal{U}$;

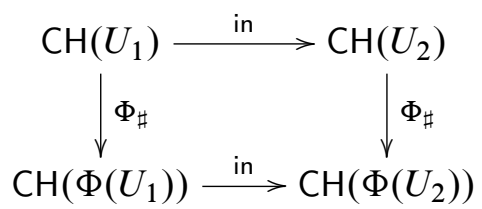

provided $U_{1} \subset U_{2}$ are domains from $\mathcal{U}$;

$$
(\Phi \circ \Psi)_{\sharp}=\Phi_{\sharp} \circ \Psi_{\sharp} ;
$$

for all $\Phi, \Psi \in \mathcal{G}$;

$$
\operatorname{in}_{U}=\mathbf{1} \text { and } \mathbf{1}_{\sharp}=\mathbf{1},
$$

where in $U$ is the natural inclusion $U \rightarrow U$.

In a more formal language, $\mathrm{CH}$ is a $\mathcal{G}$-functor from the category, whose objects are domains from $\mathcal{U}$ and morphisms correspond to inclusions, to the category of $\mathbb{Z}$-graded vector spaces over $\mathbb{Z}_{2} \cdot{ }^{3}$

\footnotetext{
${ }^{3}$ Let $\mathcal{U}$ be a category, and a group $\mathcal{G}$ acts on $\mathcal{U}$ by functors. A $\mathcal{G}$-functor is a functor $F$ from $\mathcal{U}$ to another category, and a family of natural transformations $g_{\sharp}: F \rightarrow F \circ g, g \in \mathcal{G}$ such that $(g h)_{\sharp}=g_{\sharp} \circ h_{\sharp}$ for all $g, h \in \mathcal{G}$. The terminology is borrowed from Jackowski and Słomińska [33].
} 
A typical application of contact homology to non-squeezing is given in the following statement:

Proposition 1.26 Let $U_{1}, U_{2}$ and $W$ be domains from $\mathcal{U}$ such that Closure $\left(U_{1}\right) \subset W$ and Closure $\left(U_{2}\right) \subset W$. Assume that that the inclusion morphism $\mathrm{CH}_{k}\left(U_{1}\right) \rightarrow \mathrm{CH}_{k}(W)$ does not vanish, while the inclusion morphism $\mathrm{CH}_{k}\left(U_{2}\right) \rightarrow \mathrm{CH}_{k}(W)$ vanishes for some $k \in \mathbb{Z}$. Then $U_{1}$ cannot be mapped into $U_{2}$ by a contactomorphism $\Phi \in \mathcal{G}$ with $\Phi(W)=W$.

Proof Assume on the contrary that there exists a contactomorphism $\Phi \in \mathcal{G}$ such that $\Phi(W)=W$ and $\Phi\left(U_{1}\right) \subset U_{2}$. Then the diagrams (5) and (6) yield the following commutative diagram:

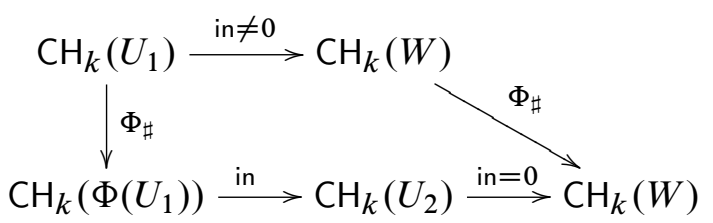

But $\Phi_{\sharp}$ is an isomorphism, and we get a contradiction which proves the desired nonsqueezing.

Our next result relates contact homology of a split domain $\widehat{D}=D \times S^{1}$, where $D$ is a starshaped domain of $M$, with filtered symplectic homology $\mathrm{SH}_{*}(D)$. We use a version of symplectic homology associated to negative compactly supported Hamiltonians as in $[3 ; 10 ; 25]$, see Section 4 below for the definition and conventions about the grading.

Theorem 1.27 Assume that the characteristic foliation of $\partial D$ has no closed orbits $\gamma$ with $\int_{\gamma} \alpha=1$. Then

$$
\mathrm{CH}_{*}(\widehat{D})=\mathrm{SH}_{*}^{(-\infty ;-1)}(D) .
$$

Moreover, the above correspondence between symplectic and contact homology is functorial in the sense that it commutes with the morphisms induced by inclusions and symplectic/contact diffeomorphisms of domains.

We restate and prove this result in Section 5.1 below (see Theorem 5.3 and Proposition 5.6). Theorem 1.27 enables us to calculate contact homology for domains of the form $\widehat{E}(N, R)$, where $E(N, R)$ is an ellipsoid given by

$$
E(N, R):=\left\{\pi\left|z_{1}\right|^{2}+\frac{\pi}{N} \sum_{i=2}^{n}\left|z_{i}\right|^{2}<R\right\}, N \in \mathbb{N}, \frac{1}{R} \notin \mathbb{N}
$$


Put

$$
k(N, R)=-2\left[\frac{1}{R}\right]-2(n-1)\left[\frac{1}{N R}\right],
$$

where $[s]$ stands for the integer part of a positive real number $s$.

Theorem 1.28 $\mathrm{CH}_{i}(\widehat{E}(N, R))=\mathbb{Z}_{2}$ for $i=k(N, R)$ and $\mathrm{CH}_{i}(\widehat{E}(N, R))=0$ for all $i \neq k(N, R)$.

Using the correspondence between contact and symplectic homologies, it is easy to calculate the morphisms induced by natural inclusions of balls (that is $N=1$ ):

Theorem 1.29 Assume that

$$
\frac{1}{k}<R_{1}<R_{2}<\frac{1}{k-1}
$$

for some $k \in \mathbb{N}$. Then the inclusion $B^{2 n}\left(R_{1}\right) \hookrightarrow B^{2 n}\left(R_{2}\right)$ induces an isomorphism of contact homologies.

Theorems 1.28 and 1.29 are proved in Section 5.2 below. Now we are ready to prove Non-Squeezing Theorem 1.2.

Proof of Theorem 1.2 Without loss of generality assume that $R_{2}<m<R_{1}$ for some $m \in \mathbb{N}$.

First of all we reduce the problem to the case $m=1$ as follows. Assume on the contrary that there exists a contactomorphism $\Phi \in \mathcal{G}$ so that

$$
\Phi\left(\operatorname{Closure}\left(\widehat{B}^{2 n}\left(R_{1}\right)\right)\right) \subset \widehat{C}^{2 n}\left(R_{2}\right) .
$$

Define the $m$-fold covering

$$
\tau: \mathbb{R}^{2 n} \times S^{1} \rightarrow \mathbb{R}^{2 n} \times S^{1},(z, t) \mapsto(\sqrt{m} z, m t) .
$$

Observe that $\tau^{*}(d t-\alpha)=m(d t-\alpha)$, and therefore

$$
\tau_{*} \xi=\xi
$$

Furthermore,

$$
\tau^{-1}\left(\widehat{B}^{2 n}\left(R_{1}\right)\right)=\widehat{B}^{2 n}\left(R_{1} / m\right) \text { and } \tau^{-1}\left(\widehat{C}^{2 n}\left(R_{2}\right)\right)=\widehat{C}^{2 n}\left(R_{2} / m\right) .
$$

Recall that the diffeomorphism $\Phi$ is compactly supported. In particular, $\Phi$ acts trivially on the fundamental group of $\mathbb{R}^{2 n} \times S^{1}$, and the complement to the support is connected. Thus $\Phi$ admits a unique lift (with respect to $\tau$ ) to a compactly supported 
diffeomorphism of the covering space. In view of (11) this lift, denoted $\Phi^{\prime}$, is a contactomorphism.

Summing up, passing to the covering space, we get that

$$
\Phi^{\prime}\left(\operatorname{Closure}\left(\widehat{B}^{2 n}\left(R_{1} / m\right)\right)\right) \subset \widehat{C}^{2 n}\left(R_{2} / m\right)
$$

where $\Phi^{\prime} \in \mathcal{G}$ and $R_{2} / m<1<R_{1} / m$. This completes the reduction to the case $m=1$.

In view of the reduction above we assume that $R_{2}<1<R_{1}$. Furthermore, let us suppose that $1 / R_{2} \notin \mathbb{Z}$. It suffices to show that $\widehat{B}^{2 n}\left(R_{1}\right)$ cannot be mapped by a contactomorphism $\Phi \in \mathcal{G}$ into domain $\widehat{E}\left(N, R_{2}\right)$ with $N$ large enough, which corresponds to a "long" ellipsoid, so that $\Phi$ keeps invariant the domain $\widehat{B}^{2 n}\left(R_{3}\right)$ with large $R_{3}$. Using Theorems 1.28 and 1.29 we see that $\mathrm{CH}_{0}\left(\widehat{B}^{2 n}\left(R_{1}\right)\right)=\mathrm{CH}_{0}\left(\widehat{B}^{2 n}\left(R_{3}\right)\right)=\mathbb{Z}_{2}$ and, moreover, the natural inclusion induces an isomorphism in contact homology. Here we used that $R_{3}>R_{1}>1$. On the other hand $\mathrm{CH}_{0}\left(\widehat{E}\left(N, R_{2}\right)\right)=0$ since $R_{2}<1$. The desired result follows now from Proposition 1.26.

Proof of Theorem 1.5 Exactly as in the previous proof, we reduce the problem to the case $m=1$. Assume that

$$
\frac{1}{k+1}<R_{2}<\frac{1}{k}<R_{1}<R_{3}<\frac{1}{k-1} .
$$

We have to show that $\widehat{B}^{2 n}\left(R_{1}\right)$ cannot be mapped into $\widehat{B}^{2 n}\left(R_{2}\right)$ by a contactomorphism from $\mathcal{G}$ which keeps invariant the domain $\hat{B}^{2 n}\left(R_{3}\right)$. Put $N=-2 n(k-1)$. Using Theorems 1.28 and 1.29 we see that

$$
\mathrm{CH}_{N}\left(\widehat{B}^{2 n}\left(R_{1}\right)\right)=\mathrm{CH}_{N}\left(\widehat{B}^{2 n}\left(R_{3}\right)\right)=\mathbb{Z}_{2},
$$

and moreover, the natural inclusion induces an isomorphism in contact homology. On the other hand $\mathrm{CH}_{N}\left(\widehat{B}^{2 n}\left(R_{2}\right)\right)=0$. The desired result follows now from Proposition 1.26 .

\subsection{A guide for the reader}

In the next diagram we present logical interrelations between the main results, methods and phenomena described above. We abbreviate $\mathrm{PC}$ for positive contractible and $\mathrm{CH}$ for contact homology. The arrows are labeled by the numbers of the corresponding theorems and/or propositions. 


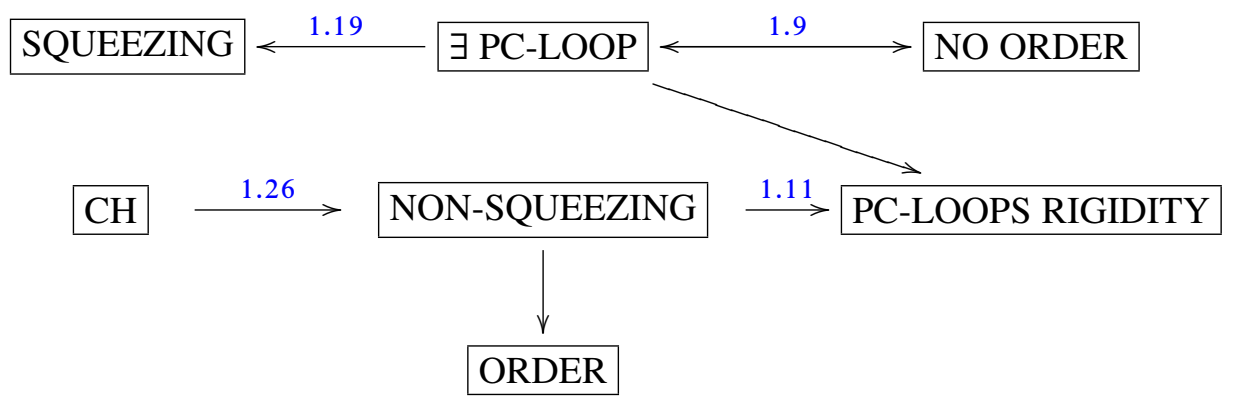

The rest of the paper is organized as follows:

In Section 2 we continue our discussion started in Section 1.7 where we suggest to use loops of contactomorphisms as a squeezing tool for contact domains. In particular, we prove Theorem 1.19 and generalize (and demystify) the formulas which appeared in the proof of Proposition 1.24.

In Section 3 we prove Theorem 1.16 which states that the ideal contact boundary of the $n$-stabilization of any Liouville manifold is non-orderable, provided that $n \geq 2$. To do this, we present a rather explicit construction of a positive contractible loop of contactomorphisms. Analyzing an explicit homotopy of our positive loop to the constant loop, we prove Theorem 1.11(ii). Finally, we prove Theorem 1.6 on negligibility of the cylinder.

To prove our results on contact non-squeezing and orderability we shall need to understand the relation between cylindrical contact homology, which is a special case of the symplectic field theory, and Hamiltonian Floer homology. In Section 4 we introduce a version of Floer homology which contains both theories as its special cases.

In Section 5 we complete the proof of several "hard" results stated in Section 1. First of all, we express contact homology of the prequantization of a symplectic domain in terms of its filtrated symplectic homology, see Theorem 5.3 and Proposition 5.6 below which together form a slightly more explicit version of Theorem 1.27. Our approach is based on generalized Floer homology theory developed in Section 4. We apply this result to various calculations with contact homology. In particular, we prove Theorems 1.28 and 1.29 on contact homology of prequantizations of ellipsoids and balls in $\mathbb{R}^{2 n}$. Furthermore, we study contact homology of prequantizations of unit ball bundles of closed manifolds in terms of cohomology of free loop spaces. As a result, we prove Theorem 1.18 on orderability of spaces of contact elements.

In Section 6 we touch miscellaneous topics related to the geometry of contact domains and transformations: We discuss (non)-squeezing of contact domains from the viewpoint 
of quantum mechanics. Then we speculate on links between orderability and fillability of contact manifolds. Finally, we introduce a canonical semigroup associated to a contact manifold. This semigroup carries footprints of a partial order even when the manifold is non-orderable.

\section{Loops of contactomorphisms as a squeezing tool}

Here we prove Theorem 1.19 by filling the gaps in the sketch presented in Section 1.7 above, and generalize the formulas which appeared in the proof of Proposition 1.24.

\subsection{Proof of Theorem 1.19}

Denote by $e_{t}$ the Hamiltonian flow of $E$ on $M_{*}$. Denote by $\epsilon$ the path of contactomorphisms of $P$ corresponding to $e_{t / R}, t \in[0 ; 1]$. With this notation we have $A(R)=U(\epsilon)$. Fix $\delta>0$ small enough so that

$$
F_{1}(z, t)>\delta R^{-1} E(z) \text { and } F_{s}(z, t)>-(1-\delta) \mu E(z)
$$

for all $z \in M_{*}, t \in S^{1}, s \in[0,1]$. Choose a non-decreasing function $\tau:[0,1] \rightarrow[0,1]$ such that $\tau \equiv 0$ near $0, \tau \equiv 1$ near 1 and $\tau^{\prime}(t)<1+\delta$ for all $t \in[0,1]$. Put

$$
\theta(t)=(1+\delta) t-\delta \tau(t)
$$

Note that

$$
\theta^{\prime}(t) \equiv 1+\delta \text { for } t \text { near } 0 \text { and } 1 \text {, and } \theta^{\prime}(t) \geq 1-\delta^{2}
$$

for all $t$.

Let us first prove part (ii) of the theorem. Our strategy is as follows. We describe a homotopy $\epsilon_{S}, s \in[-1,1]$, of the path $\epsilon=\epsilon_{-1}$ with fixed end-points such that

$$
U\left(\epsilon_{S}\right) \subset A(\rho)
$$

for all $s$ and Closure $\left(U\left(\epsilon_{1}\right)\right) \subset A(R)$. The special attention is paid to the fact that the paths $\epsilon_{S}$ have to be generated by 1-periodic in time Hamiltonians: the functions $\tau$ and $\theta$ are designed just to guarantee time-periodicity.

Each $\epsilon_{s}$ corresponds to a path $\left\{h_{s, t}\right\}$ of $\mathbb{R}_{+}$-equivariant symplectomorphisms of $M_{*}$ generated by contact Hamiltonians $H_{S}(z, t)$. These symplectomorphisms are defined as follows. 
Step $1 s \in[-1,0]$

This part of our homotopy consists of time-reparameterizations of $\left\{e_{t / R}\right\}$. Put

$$
\kappa(s, t)=(-s t+(s+1) \theta(t)) R^{-1},
$$

and set $h_{s, t}=e_{\kappa(s, t)}$. Then

$$
H_{S}(z, t)=\left(-s+(s+1) \theta^{\prime}(t)\right) R^{-1} E(z) .
$$

It follows from formula (13) that $H_{S}(z, t)$ descends to $M_{*} \times S^{1}$ and inclusion (14) holds provided

$$
\delta^{2}<1-\frac{R}{\rho}
$$

Step $2 s \in[0,1]$

Put $h_{s, t}=e_{\theta(t) / R} \circ f_{\tau(t), s}$. The corresponding Hamiltonians are given by

$$
H_{S}(z, t)=\theta^{\prime}(t) R^{-1} E(z)+\tau^{\prime}(t) F_{S}\left(e_{-\theta(t) / R^{z}}, \tau(t)\right) .
$$

Taking into account that $\tau^{\prime}(t) \equiv 0$ and $\theta^{\prime}(t) \equiv 1+\delta$ for $t$ near 0 and 1 we get that $H_{S}$ descend to Hamiltonians on $M_{*} \times S^{1}$. Combining the first inequality in (12) with the energy conservation law for $\left\{e_{t}\right\}$ we get that

$$
\begin{gathered}
H_{1}(z, t)>R^{-1} \theta^{\prime}(t) E(z)+\tau^{\prime}(t) R^{-1} \delta \cdot E\left(e_{\left.-\theta(t) / R^{z}\right)}\right. \\
=R^{-1} E(z)\left(\theta^{\prime}(t)+\delta \tau^{\prime}(t)\right)=R^{-1}(1+\delta) E(z) .
\end{gathered}
$$

Thus

$$
U\left(\epsilon_{1}\right) \subset A\left(\frac{R}{1+\delta}\right) .
$$

It remains to verify inclusion (14). Combining the second inequality in (12) with the energy conservation law for $\left\{e_{t}\right\}$ we get that

$$
H_{s}(z, t)>\left(R^{-1} \theta^{\prime}(t)-\mu(1-\delta) \tau^{\prime}(t)\right) E(z) .
$$

But $\tau^{\prime}(t)<1+\delta$ by assumption and $\theta^{\prime}(t) \geq 1-\delta^{2}$ due to formula (13), and hence

$$
H_{S}(z, t) \geq\left(R^{-1}-\mu\right)\left(1-\delta^{2}\right) E(z) .
$$

This yields inclusion (14) provided that

$$
\delta^{2}<1-\frac{1}{\rho\left(R^{-1}-\mu\right)} .
$$

This completes the proof of part (ii) of the theorem. 
In order to prove part (i) we put $\rho=+\infty$ and note that the restrictions (15) and (17) on $\delta$ read $\delta<1$. Therefore, the choice of $\delta$ is governed by inequalities (12). Assume that

$$
F_{1}(z, t)>c E(z) \text { and } F_{s}(z, t)>-(1-c) \mu E(z)
$$

for some $c \in(0 ; 1)$. Then the arguments above will work with $\delta=\min (c R, c)$. Put $\gamma=\min (c, c \mu)$. Since $R \mu<1$ we have $\delta \geq \gamma R$. Thus inclusion (16) yields

$$
U\left(\epsilon_{1}\right) \subset A\left(\frac{R}{1+\gamma R}\right),
$$

which proves part (i) of the theorem.

\subsection{Loops in Cont $_{0}(P)$ as contactomorphisms of $S P \times S^{1}$}

Let $(P, \eta)$ be a contact manifold. Write $\alpha$ and $L$ for the Liouville form and the Liouville vector field on $S P$, respectively. Consider a contact manifold $\left(S P \times S^{1}, \xi\right)$ where $\xi=\operatorname{Ker}(d t-\alpha)$. In this section we study a general construction which relates loops in Cont $_{0}(P)$ and contact embeddings of domains in $S P \times S^{1}$. This construction is behind the explicit formula in the proof Proposition 1.24 of the Introduction. Let $\left\{h_{t}\right\}, t \in S^{1}, h_{0}=\mathbf{1}$ be any loop of equivariant symplectomorphisms of $S P$ generated by a contact Hamiltonian $H: S P \times S^{1} \rightarrow \mathbb{R}$. In what follows the expression of the form

$z / c$ with $z \in S P$ and $c \in \mathbb{R}_{+}$is understood in the sense of the canonical $\mathbb{R}_{+}$-action on $S P$.

Proposition 2.1 The map

$$
\Psi(z, t)=\left(\frac{h_{t} z}{1+H\left(h_{t} z, t\right)}, t\right)
$$

is a contact embedding of the domain $\left\{1+H\left(h_{t} z\right)>0\right\} \subset S P \times S^{1}$ into $S P \times S^{1}$.

Proof Recall that for any contact Hamiltonian $H$ on $S P$ we have

$$
\alpha(\operatorname{sgrad} H)=H .
$$

This is simply the Euler formula for homogeneous functions. Furthermore, $\alpha(L)=0$. We denote by $A_{c}: S P \rightarrow S P$ the action of $c \in \mathbb{R}_{+}$on $S P$. Note that $A_{c}^{*} \alpha=c \alpha$. Put

$$
c=\left(1+H\left(h_{t} z, t\right)\right)^{-1} .
$$

For every tangent vector $v \in T_{z}(S P)$

$$
\Psi_{*} v=\mathrm{const} \cdot L+A_{c *} h_{t *} v,
$$


hence

$$
(d t-\alpha)\left(\Psi_{*} v\right)=c(d t-\alpha)(v)
$$

Furthermore,

$$
\Psi_{*} \frac{\partial}{\partial t}=\text { const } \cdot L+c \cdot \operatorname{sgrad} H_{t}+\frac{\partial}{\partial t},
$$

where $H_{t}(z):=H(z, t)$. Hence

$$
\begin{aligned}
(d t-\alpha)\left(\Psi_{*} \frac{\partial}{\partial t}\right) & =1-c \alpha\left(\operatorname{sgrad} H_{t}\right) \\
& =1-c H\left(h_{t} z, t\right) \\
& =c(d t-\alpha)\left(\frac{\partial}{\partial t}\right) .
\end{aligned}
$$

Formulas (18) and (19) yield $\Psi^{*}(d t-\alpha)=c(d t-\alpha)$ which means that $\Psi$ is a contactomorphism on its domain of definition.

Example 2.2 When $(P, \eta)$ is the standard contact sphere $S^{2 n-1}$ and $h_{t}(z)=e^{2 \pi i N t}$ then in view of our identification of $S P$ with $\mathbb{C}^{n} \backslash 0$ the map $\Psi$ from Proposition 2.1 takes the form

$$
\Psi_{N}(z, t)=\left(\frac{e^{2 \pi i N t} z}{\sqrt{1+\pi N|z|^{2}}}, t\right),
$$

which coincides with the formula (4) in the proof of Proposition 1.24.

\section{Non-orderability of stabilizations}

In this section we prove Theorem 1.16 which states that the ideal contact boundary $P_{\text {stab }}$ of the $n$-stabilization $M_{\text {stab }}=M \times \mathbb{C}^{n}$ of any Liouville manifold $(M, \omega, L)$ is non-orderable if $n \geq 2$. To do this, we present a rather explicit construction of a positive contractible loop of contactomorphisms of $P_{\text {stab }}$. The desired result then follows from Proposition 1.9. When $M$ is a point, $P_{\text {stab }}$ is simply the standard $(2 n-1)$-dimensional contact sphere. Analyzing an explicit homotopy of our positive loop to the constant loop, we prove Theorem 1.11(ii). Finally, we combine our squeezing techniques with the construction from Section 2.2 and get Theorem 1.6 on negligibility of the cylinder.

\subsection{An ambient isotopy}

We start with a version of the ambient isotopy theorem in contact geometry which will be used below. Let $(P, \eta)$ be a closed contact manifold with a co-oriented contact structure, and let $\Sigma \subset S P$ be a starshaped hypersurface. 
Consider a Hamiltonian isotopy $h_{t}: W \rightarrow S P, t \in[0,1], h_{0}=\mathbf{1}$, of a neighborhood $W$ of $\Sigma$ in $S P$. We denote by $H_{t}$ the Hamiltonian function generating $h_{t}$, by $\sigma_{t}$ the characteristic foliation on $\Sigma_{t}=h_{t}(\Sigma)$, and write $\operatorname{sgrad} H$ for the Hamiltonian vector field of a function $H$ on $S P$.

Lemma 3.1 (Ambient isotopy) Assume that hypersurfaces $\Sigma_{t}$ are starshaped for all $t$. Then there exists a path $\left\{f_{t}\right\}, t \in[0,1]$, of $\mathbb{R}_{+}$-equivariant symplectomorphisms of $S P$ with $f_{0}=1$ such that $f_{t}(\Sigma)=\Sigma_{t}$ and $\operatorname{sgrad} F_{t}-\operatorname{sgrad} H_{t}$ is tangent to $\sigma_{t}$, where we denote by $F_{t}$ the Hamiltonian which generates the path $f_{t}$.

Let $\beta$ be the contact form on $S P$ whose graph equals $\Sigma$. In what follows we need a formula for the Hamiltonian vector field of a function $H$ in canonical local coordinates $(x, u) \in P \times \mathbb{R}_{+}$associated to $\beta$, see Section 1.3 above. We assume that $H=H(x, u)$ is defined in a neighborhood of $\Sigma$, that is in the set $\{x \in P,|u-1|<\delta\}$. For every $x \in P$ and $u \in(1-\delta ; 1+\delta)$ there exists a unique vector $\operatorname{sgrad}_{\beta} H \in \eta(x)$ such that $d \beta\left(v, \operatorname{sgrad}_{\beta} H\right)=-\left.d H\right|_{(x, u)}(v)$ for all $v \in \eta(x)$. Let $R$ be the Reeb vector field of $\beta$. A straightforward calculation shows that

$$
\operatorname{sgrad} H=-d H(R) \frac{\partial}{\partial u}+u^{-1} \operatorname{sgrad}_{\beta} H+\frac{\partial H}{\partial u} R .
$$

Proof of Lemma 3.1 Let $H_{t}: h_{t}(W) \rightarrow \mathbb{R}$ be the Hamiltonian function generating $h_{t}$. Denote by $F_{t}$ the $\mathbb{R}_{+}$-equivariant Hamiltonian which equals $H_{t}$ on $\Sigma_{t}$. It generates a path $f_{t}$ of equivariant symplectomorphisms. Note that, for fixed $t$, in canonical coordinates near $\Sigma_{t}$ we have $F_{t}(x, u)=u H_{t}(x, 1)$. Using the fact that $\Sigma_{t}=\{u=1\}$ and formula (20) we get that at each point of $\Sigma_{t}$ the difference $\operatorname{sgrad} F_{t}-\operatorname{sgrad} H_{t}$ is tangent to $\Sigma_{t}$. Since this is true for all $t \in[0,1]$, we conclude that $f_{t}(\Sigma)=\Sigma_{t}$. Note that the two first terms in formula (20) depend only on the restriction of the Hamiltonian to the hypersurface and, therefore, these terms coincide for $F_{t}$ and $H_{t}$. On the other hand, the third term is tangent to $\sigma_{t}$, and hence so is $\operatorname{sgrad} F_{t}-\operatorname{sgrad} H_{t}$.

\subsection{Fundamental Lemma on unitary transformations}

In this section we consider unitary transformations of $\mathbb{C}^{n}$ as symplectomorphisms. In particular, every path in $U(n)$ is generated by unique quadratic Hamiltonian. We represent a point $z \in \mathbb{C}^{n}$ as $z=\left(z_{1}, \ldots, z_{n}\right)=\left(z_{1}, w\right)$, where $w=\left(z_{2}, \ldots, z_{n}\right)$, and introduce radial coordinates $\rho_{j}=\pi\left|z_{j}\right|^{2}$ for $j=1, \ldots, n$ and $\varrho=\rho_{2}+\ldots+\rho_{n}$.

The following loop, which lies in $S U(n)$ (and hence is necessarily contractible) is an important character of our story:

$$
f_{t}:\left(z_{1}, w\right) \rightarrow\left(e^{2 \pi i(n-1) t} z_{1}, e^{-2 \pi i t} w\right) .
$$


Lemma 3.2 (Fundamental Lemma) The loop $\left\{f_{t}\right\}$ given by formula (21) admits a homotopy $\left\{f_{t}^{(s)}\right\}, f_{t}^{(1)}=f_{t}, f_{t}^{(0)} \equiv \mathbf{1}$ through loops on $S U(n)$ based at $\mathbf{1}$ to the constant loop so that the corresponding Hamiltonians $F^{(s)}(z, t)$ satisfy

$$
F^{(s)}\left(f_{t}^{(s)} z, t\right) \geq-\varrho
$$

for all $s \in[0 ; 1], t \in S^{1}, z \in \mathbb{C}^{n} \backslash\{0\}$.

Proof Denote by $b_{j, t}$ the unitary transformation which preserves all the coordinates besides the $j$-th one, and multiplies the $j$-th coordinate by $e^{2 \pi i t}$. Let $I_{j}^{(s)}, j \geq 2, s \in$ $[0, \pi / 2]$, be the unitary transformation which preserves all the coordinates besides the first and the $j$-th ones, and in the $\left(z_{1}, z_{j}\right)$-plane its action is given by

$$
\left(z_{1}, z_{j}\right) \mapsto\left(\cos s \cdot z_{1}-\sin s \cdot z_{j}, \sin s \cdot z_{1}+\cos s \cdot z_{j}\right) .
$$

Put

$$
h_{j, t}^{(s)}=I_{j}^{(s)} b_{j, t}\left(I_{j}^{(s)}\right)^{-1} b_{j,-t}
$$

and

$$
f_{m, t}^{(s)} z=h_{2, t}^{(s)} \circ \ldots \circ h_{m, t}^{(s)}, \quad m=2, \ldots, n .
$$

For $m=n$ this family of loops gives a homotopy of $\left\{f_{t}\right\}$ to the constant loop. To verify that this homotopy satisfies inequality (22) we prove the following more general inequality for every $m=2, \ldots, n$ :

Claim The Hamiltonian $F_{m}^{(s)}$ generating the loop $f_{m, t}^{(s)}$ satisfies

$$
F_{m}^{(s)}\left(f_{m, t}^{(s)} z, t\right) \geq-\left(\rho_{2}+\ldots+\rho_{m}\right) .
$$

In the proof of the claim we use the following fact:

Suppose that Hamiltonian $G(z, t)$ generates a flow $g_{t}$. Then the Hamiltonian $-G\left(g_{t} z, t\right)$ generates the flow $g_{t}^{-1}$.

We use notation $(u)_{j}$ for the $j$-th coordinate of a vector $u \in \mathbb{C}^{n}$. Write $H_{j}^{(s)}(z, t)$ for the Hamiltonian generating $h_{j, t}^{(s)}$. Calculating, we get that

$$
H_{j}^{(s)}\left(h_{j, t}^{(s)} z, t\right)=-\pi\left|z_{j}\right|^{2}+\pi\left|\left(\left(I_{j}^{(s)}\right)^{-1} b_{j,-t} z\right)_{j}\right|^{2} \geq-\pi\left|z_{j}\right|^{2} .
$$

Another observation used below is that

$$
\left(h_{j, t}^{(s)} z\right)_{l}=z_{l} \text { for } l \neq 1, j .
$$


Proof of the Claim We use induction in $m$. The case $m=2$ immediately follows from inequality (24). Let us verify the induction step $m \mapsto m+1$. Note that $f_{m+1, t}^{(s)}=$ $f_{m, t}^{(s)} \circ h_{m+1, t}^{(s)}$. Hence, putting $y=h_{m+1, t}^{(s)} z$, we get

$$
F_{m+1, t}^{(s)}\left(f_{m+1, t}^{(s)} z, t\right)=H_{m+1}^{(s)}\left(h_{m+1, t}^{(s)} z, t\right)+F_{m}^{(s)}\left(f_{m, t}^{(s)} y, t\right) .
$$

Applying the induction hypothesis and inequality (24) we get that the left hand side of (26) is greater than or equal to

$$
C:=-\pi\left|z_{m+1}\right|^{2}-\pi \sum_{j=2}^{m}\left|(y)_{j}\right|^{2} .
$$

Applying (25) we see that $(y)_{j}=z_{j}$ for $j=2, \ldots, m$. Hence

$$
C=-\pi\left(\rho_{2}+\ldots+\rho_{m}\right),
$$

and inequality (23) follows.

This completes the proof of the Claim, and hence of Lemma 3.2.

\subsection{Some preparations}

Let $(M, \omega, L)$ be a Liouville manifold with the ideal contact boundary $(P, \eta)$. We fix a contact form on $P$ and equip the symplectization $S P$ with coordinates $(x, u) \in P \times \mathbb{R}_{+}$ as in Section 1.3. Thus we have decomposition (2)

$$
M=\left(P \times \mathbb{R}_{+}\right) \sqcup \operatorname{Core}(M) .
$$

We extend the function $u$ by 0 to $\operatorname{Core}(M)$. As before, we introduce the functions $\rho_{1}=\pi\left|z_{1}\right|^{2}$ and $\varrho=\sum_{j=2}^{n} \pi\left|z_{j}\right|^{2}$ on $\mathbb{C}^{n}$, and denote $\rho:=\rho_{1}+\varrho$.

In what follows we have to work with starshaped domains and hypersurfaces in the stabilization $M_{\text {stab }}=M \times \mathbb{C}^{n}$. A user-friendly class of these objects arises, roughly speaking, as follows: Take any closed starshaped domain $D \subset \mathbb{C}^{n}$ with boundary $\Gamma=\partial D$. Then $\{u \leq 1\} \times D$ is a closed starshaped domain in $M_{\text {stab }}$ whose boundary is a starshaped hypersurface. The problem is that these objects (the domain and the hypersurface) have corners unless $M$ is a point, and hence do not fit into our setup. However, this can be easily corrected by an appropriate smoothing of corners. Below we describe this construction in a slightly more general context when the domain $D$ has the form $D=\left\{c_{1} \rho_{1}+c \varrho \leq 1\right\}$ and $\Gamma=\left\{c_{1} \rho_{1}+c \varrho=1\right\}$ with $c_{1}, c \in \mathbb{R}$. (Note that $D$ and $\Gamma$ are not necessarily compact.)

We fix a real concave function $\theta:[0,1] \rightarrow[0,1]$ (a mollifier) which equals 1 near 0 and equals 0 at 1 . We assume, in addition, that its inverse $\theta^{-1}$, which is defined near 0 , is 
flat at 0 , ie all its derivatives at 0 vanish. The graph of $\theta$ is a smoothing of the union of northern and eastern edges of the unit square on the coordinate plane. In addition, we need another technical condition. Fix a number $\lambda \in(0,1)$ which is sufficiently close to 1 and assume that

$$
\theta^{\prime}(u)<-\lambda^{2} / 4 \text { provided } \theta(u) \leq \lambda^{2} .
$$

The desired smoothing of corners gives rise to a domain

$$
D_{e x t}=\left\{c_{1} \rho_{1}+c \varrho \leq \theta(u)\right\}
$$

and to a hypersurface

$$
\Gamma_{\text {ext }}=\left\{c_{1} \rho_{1}+c \varrho=\theta(u)\right\}
$$

in $M_{\text {stab }}$. The subscript ext indicates that we extended an object defined on $\mathbb{C}^{n}$ to $M \times \mathbb{C}^{n}$. Note that with this notation the hypersurface $S_{\text {ext }}^{2 n-1}$ represents the ideal contact boundary of $M_{\text {stab }}$.

\subsection{Distinguished $\mathbb{R}_{+}$-equivariant symplectomorphism}

Consider the following subsets of $\mathbb{C}^{n}$ : the domain

$$
W:=\left\{(2 n+1) \varrho-(n-1) \rho_{1} \leq 1\right\},
$$

the ellipsoid

$$
E:=\left\{v \rho_{1}+\varrho=1\right\}
$$

and the ball

$$
B=\{\rho \leq 1\} .
$$

For $c>0$ let us denote by $Y_{c}: \mathbb{C}^{n} \rightarrow \mathbb{C}^{n}$ the shift by $c$ along the $\operatorname{Re}\left(z_{1}\right)$-axis, and write $\tilde{Y}_{c}$ for its trivial extension $\mathbf{1} \times Y_{c}$ to a diffeomorphism of $M \times \mathbb{C}^{n}$.

Lemma 3.3 There exist $c>0$ large enough and $v>0$ small enough such that all ellipsoids $Y_{S}(E), s \in[0, c]$, enclose 0 and hence are starshaped, and in addition

$$
Y_{b}(E) \subset \operatorname{Interior}(W) \text { for all } b \geq c .
$$

Proof Let us make analysis of the problem: suppose that $c$ and $v$ are as required. Take any $z=\left(z_{1}, w\right) \in E$. Put $z^{\prime}=Y_{b}(z)=\left(z_{1}+b, w\right)$ with $b \geq c$. We have to check that

$$
(n-1) \pi\left|z_{1}+b\right|^{2}>(2 n+1) \pi|w|^{2}-1 .
$$

Substituting $\pi|w|^{2}=1-\pi v\left|z_{1}\right|^{2}$ we rewrite this inequality as

$$
(n-1) \pi\left|z_{1}+b\right|^{2}+(2 n+1) v \pi\left|z_{1}\right|^{2}>2 n .
$$


Write $z_{1}=p+i q$ and observe that it suffices to achieve

$$
(n-1) \pi(p+b)^{2}+(2 n+1) v \pi b^{2}>2 n
$$

for all $b \geq c$. The absolute minimum of the function in the left hand side of this inequality equals

$$
\frac{(n-1)(2 n+1) \pi v b^{2}}{(n-1)+(2 n+1) v} .
$$

Substitute this into the left hand side of (29). Replacing $b$ by $c$ and performing an elementary algebraic manipulation we get

$$
\pi v c^{2}>\frac{2 n}{2 n+1}+v \frac{2 n}{n-1} .
$$

On the other hand, $Y_{s}(E)$ encloses 0 for all $s \leq c$ provided that

$$
\pi v c^{2}<1 \text {. }
$$

To complete the proof it remans to notice that inequalities (30) and (31) are compatible if $v$ is small enough.

Take $c$ and $v$ from the lemma and choose $\lambda \in(0,1)$ so that

$$
\pi v c^{2} \leq \lambda^{2}<1
$$

(see inequality (31) above). In what follows we assume that this number $\lambda$ appears in the condition (27) on the mollifier $\theta$.

Lemma 3.4 All domains $\tilde{Y}_{s}\left(E_{\text {ext }}\right), s \in[0, c]$, are starshaped in $M_{s t a b}$, and in addition

$$
\tilde{Y}_{c}\left(E_{\text {ext }}\right) \subset \operatorname{Interior}\left(W_{\text {ext }}\right) .
$$

Proof Write $z_{j}=p_{j}+i q_{j}$, and note that the Liouville field on $M_{\text {stab }}$ can be written as

$$
L=\partial_{u} \oplus \frac{1}{2} \sum_{j=1}^{n}\left(p_{j} \frac{\partial}{\partial p_{j}}+q_{j} \frac{\partial}{\partial q_{j}}\right) .
$$

Choose $s \in[0, c]$. The equation of $\tilde{Y}_{s}\left(E_{\text {ext }}\right)$ is

$$
F(p, q, u):=\pi v\left(p_{1}-s\right)^{2}+\pi v q_{1}^{2}+\pi \cdot \sum_{j=2}^{n}\left(p_{j}^{2}+q_{j}^{2}\right)-\theta(u)=0 .
$$


We claim that $d F(L)>0$ at every point of the hypersurface $\tilde{Y}_{S}\left(E_{\text {ext }}\right)$. Indeed, calculating $d F(L)$ at such a point we get

$$
d F(L)=\pi v s\left(p_{1}-s\right)+\theta(u)-\theta^{\prime}(u)
$$

On $\tilde{Y}_{s}\left(E_{\text {ext }}\right)$ we have $\left|p_{1}-s\right| \leq \sqrt{\theta(u) /(\pi v)}$. Thus inequality (32) yields

$$
d F(L) \geq \theta(u)-\lambda \sqrt{\theta(u)}-\theta^{\prime}(u)
$$

The positivity of the right hand side, say $A$, of this expression follows from assumption (27) on the mollifier $\theta$. Indeed, denote by $I$ the segment

$$
\left\{u: \theta(u) \leq \lambda^{2}\right\}
$$

For $u \notin I$ we have $\theta(u)-\lambda \sqrt{\theta(u)}>0$ and $\theta^{\prime}(u) \leq 0$ so $A>0$. On $I$ we have $\theta(u)-\lambda \sqrt{\theta(u)} \geq-\lambda^{2} / 4$ and $-\theta^{\prime}(u)>\lambda^{2} / 4$. Thus again $A>0$.

The "singular" case when $u=1$ (and so $\theta^{\prime}(u)$ is not defined) can be easily checked separately. The claim follows.

Thus $\tilde{Y}_{S}\left(E_{\text {ext }}\right), s \in[0, c]$, is an isotopy of an (obviously) starshaped hypersurface $E_{\text {ext }}$ in the class of hypersurfaces transversal to the Liouville field $L$. Therefore all these hypersurfaces are starshaped.

In order to verify inclusion (33), we use a scaling type argument: fix $u$ and put $\varrho^{\prime}=\varrho / \theta(u), \rho_{1}^{\prime}=\rho_{1} / \theta(u), c^{\prime}=c / \sqrt{\theta(u)}$. Then the desired inclusion is equivalent to inclusion (28) with $b=c^{\prime} \geq c$ since $\theta(u) \leq 1$. This completes the proof.

Applying Ambient Isotopy Lemma 3.1 we get a family $\left\{a^{(s)}\right\}, s \in[0, c]$, of equivariant symplectomorphisms of $M_{\text {stab }}$ such that $a^{(s)}\left(E_{\text {ext }}\right)=\tilde{Y}_{s}\left(E_{\text {ext }}\right)$ for all $s \in[0, c]$. Symplectomorphism $a:=a^{(c)}$ plays an important role below. 
Lemma 3.5 The equivariant symplectomorphism $a$ satisfies the following conditions:

$$
\begin{gathered}
a\left(\partial B_{\text {ext }}\right) \subset \operatorname{Interior}\left(W_{\text {ext }}\right) ; \\
a^{*} u=u, \quad a^{*} \varrho=\varrho .
\end{gathered}
$$

Proof The property (34) is straightforward. Let us verify formulas (35). Note that the characteristic foliation $\sigma_{0}$ of $E_{s t a b}$ is generated by

$$
v \cdot \operatorname{sgrad}_{\mathbb{C}^{n}} \rho_{1}+\operatorname{sgrad}_{\mathbb{C}^{n} \varrho}-\theta^{\prime}(u) \operatorname{sgrad}_{M} u .
$$

The functions $u$ and $\varrho$ are constant along $\sigma_{0}$, and, moreover, the shifts $\tilde{Y}_{s}$ preserve them. Thus, using Ambient Isotopy Lemma 3.1, we get $u\left(a^{(s)} x\right)=u(x)$ and $\varrho\left(a^{(s)} x\right)=\varrho(x)$ for all $x \in E_{s t a b}$. In view of the homogeneity of $a$ we conclude (35).

\subsection{The main construction}

Convention All transformations $g$ from $S U(n)$ smoothly extend to $M \times \mathbb{C}^{n}$ by $\mathbf{1} \times g$. We denote the extensions by the same letter as the original transformations.

Put $e_{t} z:=e^{2 \pi i t} z$. Let $f_{t}$ be the loop of unitary transformations constructed in Fundamental Lemma 3.2. Let $a$ be the distinguished $\mathbb{R}_{+}$-equivariant symplectomorphism of $M_{\text {stab }}$ constructed in Section 3.4.

Theorem 3.6 The loop

$$
\varphi_{t}:=e_{-t} f_{3 t} a e_{t} a^{-1}
$$

is a positive contractible loop of $\mathbb{R}_{+}$-equivariant symplectomorphisms of

$$
S P_{s t a b}=M_{\text {stab }} \backslash \operatorname{Core}\left(M_{\text {stab }}\right) \text {. }
$$

In view of Proposition 1.9 this yields Theorem 1.16. In the case of the standard contact 3-sphere somewhat similar positive contractible loops can be defined by an explicit analytic formula. Furthermore, "a cousin" of the distinguished map $a$ admits a transparent geometric meaning. We refer to Remark 8.2 in Appendix B for the details.

Proof Let $f_{t}$ and $f_{t}^{(s)}$ be the loop of unitary transformations and its homotopy from Fundamental Lemma 3.2, and let $F(z)$ and $F^{(s)}(z, t)$ be the corresponding Hamiltonians considered as functions on $M \times \mathbb{C}^{n}$.

(1) Put $g_{t}:=f_{t} a e_{t} a^{-1}$ and $g_{t}^{(s)}:=f_{t}^{(s)} a e_{t} a^{-1}$, and denote by $G(x, z, t)$ and $G^{(s)}(x, z, t)$ their Hamiltonians. Here $x \in M, z \in \mathbb{C}^{n}$. We claim that

$$
G^{(s)} \geq 0
$$


and

$$
G(x, z, t)>2 n \varrho(z)
$$

for all $(x, z) \in M_{\text {stab }}, t \in S^{1}, s \in[0,1]$. Indeed,

$$
G^{(s)}(x, z, t)=F^{(s)}(z, t)+\rho\left(a^{-1}\left(x,\left(f_{t}^{(s)}\right)^{-1} z\right)\right) .
$$

Recall that $\rho=\rho_{1}+\varrho$. Put $y=\left(f_{t}^{(s)}\right)^{-1} z$. In view of $\mathbb{R}_{+}$-equivariance, it suffices to verify inequalities (36) and (37) for fixed $s$ and $t$ assuming that $(x, z)$ runs over any given starshaped hypersurface. Thus, we can assume that

$$
(x, y) \in a\left(\partial B_{\text {ext }}\right) .
$$

By Fundamental Lemma 3.2 and equations (35) we have

$$
\begin{aligned}
& G^{(s)}(x, z, t)=F^{(s)}\left(f_{t}^{(s)} y, t\right)+\rho\left(a^{-1}(x, y)\right) \geq-\varrho(y)+\rho\left(a^{-1}(x, y)\right) \\
& \geq-\varrho(y)+\varrho\left(a^{-1}(x, y)\right)=-\varrho(y)+\varrho(y)=0,
\end{aligned}
$$

which proves inequality (36).

Let us now turn to the case $s=1$. Inclusion (34) guarantees that

$$
\theta(u(x, y))+(n-1) \rho_{1}(y)-(2 n+1) \varrho(y)>0 .
$$

Note that when $s=1$ we have $y=f_{-t} z$, and hence

$$
\rho_{1}(y)=\rho_{1}(z) \text { and } \varrho(y)=\varrho(z) .
$$

Using equations (35), inequality (39) and inclusion (38) we get

$$
\begin{aligned}
G(x, z, t) & =F(y)+\rho\left(a^{-1}(x, y)\right)=(n-1) \rho_{1}(y)-\varrho(y)+\theta\left(u\left(a^{-1}(x, y)\right)\right) \\
& =(n-1) \rho_{1}(y)-\varrho(y)+\theta(u(x, y))>2 n \varrho(y)=2 n \varrho(z) .
\end{aligned}
$$

This proves inequality (37), and the claim follows.

(2) Put $h_{t}:=f_{2 t} g_{t}, h_{t}^{(s)}=f_{2 t}^{(s)} g_{t}$ and let $H(x, z, t)$ and $H^{(s)}(x, z, t)$ be corresponding Hamiltonians. We claim that

$$
H^{(s)} \geq 0
$$

and

$$
H(x, z, t)>(2 n-2) \rho(z)
$$

for all $(x, z) \in M_{\text {stab }}, t \in S^{1}, s \in[0,1]$. Indeed,

$$
H^{(s)}(x, z, t)=2 F^{(s)}(z, 2 t)+G\left(x,\left(f_{2 t}^{(s)}\right)^{-1} z, t\right) .
$$


Put $y=\left(f_{2 t}^{(s)}\right)^{-1} z$. Applying Fundamental Lemma and (37) we have

$$
H^{(s)}(x, z, t)=2 F^{(s)}\left(f_{2 t}^{(s)} y, 2 t\right)+G(x, y, t) \geq-2 \varrho(y)+2 n \varrho(y) \geq 0,
$$

which proves inequality (41).

When $s=1$ we apply formulas (40) and (37) and get

$$
H(x, z, t)=2(n-1) \rho_{1}(z)-2 \varrho(z)+G(x, y, t)>(2 n-2)\left(\rho_{1}(z)+\varrho(z)\right) .
$$

This proves inequality (42). The claim follows.

(3) Note that the loop $\left\{\varphi_{t}\right\}$ presented in the formulation of the theorem is given by $\varphi_{t}=e_{-t} h_{t}$. Let $\Phi(x, z, t)$ be the Hamiltonian of $\left\{\varphi_{t}\right\}$. Then using inequality (42) we get

$$
\Phi\left(x, e_{-t} z, t\right)=-\rho(z)+H(x, z, t)>(2 n-3) \rho(z) \geq 0
$$

and hence

$$
\Phi(x, z, t)>(2 n-3) \rho(z) \geq 0
$$

for all $(x, z) \in M_{\text {stab }}$ and $t \in S^{1}$. This proves positivity of the loop $\left\{\varphi_{t}\right\}$.

(4) Let us present a homotopy $\Delta$ of the loop $\left\{\varphi_{t}\right\}$ to the constant loop. For that purpose write

$$
\varphi_{t}=e_{-t} f_{2 t} f_{t} a e_{t} a^{-1}
$$

The homotopy $\Delta$ is given in three steps.

Step 1 Contract the term $f_{2 t}$ using homotopy $f_{2 t}^{(s)}$ given by the Fundamental Lemma and arrive to

$$
\varphi_{t}^{\prime}=e_{-t} f_{t} a e_{t} a^{-1}
$$

Step 2 Contract the term $f_{t}$ using homotopy $f_{t}^{(s)}$ given by the Fundamental Lemma and arrive to

$$
\varphi_{t}^{\prime \prime}=e_{-t} a e_{t} a^{-1}
$$

Step 3 Use the path $a^{(s)}$ joining the identity with $a$ described in Section 3.4. We get the homotopy

$$
e_{-t} a^{(s)} e_{t}\left(a^{(s)}\right)^{-1}
$$

which equals the constant loop when $s=0$.

This completes the proof.

Let us emphasize that the proof above works when $M=\{$ point $\}$ with the following modifications:

Geometry 83 Topology, Volume 10 (2006) 
- We do not need any mollifier $\theta$, and work directly with $W, E, B \subset \mathbb{C}^{n}$, omitting sub-index ext;

- The shift $\tilde{Y}_{S}$ coincides with $Y_{s}$.

Taking into account these remarks we are ready to prove Theorem 1.11(ii).

Proof of Theorem 1.11(ii) Look at the homotopy $\Delta$ of the loop $\varphi_{t}$ described in Part 4 of the proof of Theorem 3.6 above. It suffices to show that the Hamiltonians generating loops obtained in the process of this homotopy are $\geq-\pi|z|^{2}$. In Steps 1 and 2 of the homotopy we get loops $e_{-t} h_{t}^{(s)}$ and $e_{-t} g_{t}^{(s)}$, respectively. Inequalities (41) and (36) guarantee that in both cases the corresponding Hamiltonians are $\geq-\pi|z|^{2}$. In Step 3 the Hamiltonian again is $\geq-\pi|z|^{2}$. This shows that $\mu(\Delta) \leq 1$ (and in fact, one can see that $\mu(\Delta)=1$, as it should be in accordance with Theorem 1.11(i).) This completes the proof.

\subsection{Proof of Theorem 1.6}

We work in $\mathbb{C}^{n}$ with coordinates $\left(z_{1}, \ldots, z_{n}\right)$. Put $\rho_{j}=\pi\left|z_{j}\right|^{2}, \varrho=\sum_{j=2}^{n} \pi\left|z_{j}\right|^{2}$ and $\rho=\sum_{j=1}^{n} \pi\left|z_{j}\right|^{2}$. We will prove that the cylinder $C=\left\{\rho_{2}<1\right\} \subset \mathbb{C}^{n}$ is negligible.

$$
\begin{gathered}
\text { Put } D=\left\{\varrho<\frac{1}{n}\right\} \\
\text { and } \quad W=\left\{(n+1) \rho_{2}+n \rho_{3}+\ldots+n \rho_{n}-\rho_{1}<1\right\} .
\end{gathered}
$$

Introduce the loops of transformations from $S U(n)$

$$
f_{t}(z)=\left(e^{2 \pi(n-1) i t} z_{1}, e^{-2 \pi i t} z_{2}, \ldots, e^{-2 \pi i t} z_{n}\right)
$$

and

$$
g_{t}(z)=\left(e^{2 \pi i t} z_{1}, e^{-2 \pi i t} z_{2}, z_{3}, \ldots, z_{n}\right)
$$

which are generated by the Hamiltonians

$$
F(z)=(n-1) \rho_{1}(z)-\varrho(z)
$$

and

$$
G(z)=\rho_{1}(z)-\rho_{2}(z),
$$

respectively. Our main squeezing tools will be contact embeddings

and

$$
\begin{aligned}
& \Phi(z, t)=\frac{f_{t}(z)}{\sqrt{1+F(z)}} \\
& \Psi(z, t)=\frac{g_{t}(z)}{\sqrt{1+G(z)}}
\end{aligned}
$$


associated to loops $\left\{f_{t}\right\}$ and $\left\{g_{t}\right\}$, respectively, in accordance to Proposition 2.1. (The square root in these formulas reflects the fact that the natural $\mathbb{R}_{+}$-action on $\mathbb{C}^{n} \backslash\{0\}$ considered as the symplectization of $S^{2 n-1}$ is given by $z \rightarrow \sqrt{c} z$ for $c \in \mathbb{R}_{+}$.)

In view of Fundamental Lemma 3.2 the loop $\left\{f_{t}\right\}$ is homotopic to a point through loops whose Hamiltonians are $\geq-\varrho$. In particular, the embedding $\Phi: D \rightarrow V$ is well defined and contactly isotopic to the inclusion.

In view of Claim (23) inside the proof of Fundamental Lemma, the loop $\left\{g_{t}\right\}$ is homotopic to a point through loops with Hamiltonians $\geq-\rho_{2}$. In particular, the embedding $\Psi: C \rightarrow V$ is well defined and contactly isotopic to the inclusion.

Let $K \subset C$ be any compact subset. Arguing exactly as in Section 3.4 we find a contact isotopy of $K$ to its shift $Y(K)$ along the $\operatorname{Re} z_{1}$-axis such that $Y(K) \subset C \cap W$. Take $z \in C \cap W$ and put $z^{\prime}=\Psi(z)$. Then

$$
\varrho\left(z^{\prime}\right)=\frac{\varrho(z)}{1+\rho_{1}(z)-\rho_{2}(z)}<\frac{1}{n},
$$

where the equality follows from the explicit formula for $\Psi$ and the inequality follows from the definition of $W$. We conclude that $\Psi(Y(K)) \subset D$. Take now any $u \in D$ and put $u^{\prime}=\Phi(u)$. Then

$$
\rho\left(u^{\prime}\right)=\frac{\rho_{1}(u)+\varrho(u)}{1+(n-1) \rho_{1}(u)-\varrho(u)} \leq \frac{\rho_{1}(u)+\frac{1}{n}}{1+(n-1) \rho_{1}(u)-\frac{1}{n}}=\frac{1}{n-1},
$$

where the equality on the left follows from the explicit formula for $\Phi$ and the inequality follows from the definition of $D$. We conclude that

$$
\Phi(\Psi(Y(K))) \subset \widehat{B}^{2 n}\left(\frac{1}{n-1}\right) .
$$

Thus, we contactly squeezed $K$ into the set $\widehat{B}^{2 n}\left(\frac{1}{n-1}\right)$, which is negligible in view of Theorem 1.3. This shows that any compact subset of $C$ is negligible, as required.

\section{Different flavors of Floer homology}

To prove our results on contact non-squeezing and orderability we need to understand the relation between cylindrical contact homology which is a special case of the symplectic field theory (see $[17 ; 44 ; 48 ; 4]$ ), and (periodic) Floer homology for the symplectic action functional (see $[21 ; 37]$ ). In order to do that we introduce a more general version of Floer homology for Hamiltonian structures which contains both theories as its special cases. 


\subsection{Stable Hamiltonian structures}

Two differential 1-forms on a manifold are called equivalent if they differ by an exact 1 -form. We denote by $(\Theta)$ the equivalence class of a 1-form $\Theta$. The 2-form $d \Theta$ does not depend on the choice of a representative $\Theta \in(\Theta)$ and will be denoted by $d(\Theta)$. A Hamiltonian structure $\mathcal{H}$ on an odd-dimensional oriented, possibly non-compact manifold $V$ is an equivalence class $(\Theta)$ of 1 -forms such that $\Omega=d(\Theta)$ has the maximal rank. The tangent line field $\ell=\operatorname{Ker} \Omega$ is called the characteristic line field. The field $\ell$ integrates to a 1 -dimensional characteristic foliation of $\Omega$. Note that $\Omega$ defines a fiberwise symplectic structure (and hence an orientation) on the bundle $T V / \ell$. Thus the line bundle $\ell$ is equipped with an orientation. We will call characteristic any vector field $R$ which generates $\ell$ and respects its orientation.

Any co-orientable hypersurface $V$ in an exact symplectic manifold $(W, \widetilde{\Omega}=d \widetilde{\Theta})$ inherits a Hamiltonian structure $(\Theta)=\left(\left.\widetilde{\Theta}\right|_{V}\right)$. Conversely, any Hamiltonian structure $(V,(\Theta))$ embeds as a hypersurface in a symplectic manifold $(V \times(-\varepsilon, \varepsilon), d \widetilde{\Theta})$ where the form $\widetilde{\Theta}$ can be constructed as follows. Let $\lambda$ be any 1 -form which is not vanishing on $\ell$, and $s$ be the coordinate along the second factor. Then we set $\widetilde{\Theta}=\Theta+s \lambda$. Note that by Darboux' theorem the Hamiltonian structure $(V,(\Theta))$ determines the symplectic structure in its neighborhood uniquely up to a diffeomorphism fixed on $V$. We call (a germ along $V$ of ) the symplectic structure $\widetilde{\Omega}$ on $V \times(-\varepsilon, \varepsilon)$ the symplectic extension of $(V,(\Theta))$.

A Hamiltonian structure $(V,(\Theta))$ is called stable (see [32]) if its symplectic extension can be realized by a form $\tilde{\Omega}$ on $V \times(-\varepsilon, \varepsilon)$, such that all the Hamiltonian structures $\mathcal{H}_{s}, s \in(-\varepsilon, \varepsilon)$ induced on $V$ by the inclusions $V=V \times s \hookrightarrow V \times(-\varepsilon, \varepsilon)$ have the same characteristic line field $\ell$.

Proposition 4.1 A Hamiltonian structure $(V,(\Theta))$ is stable if and only if there exists a $1-$ form $\lambda$ and a characteristic vector field $R$ such that

$$
\lambda(R)=1 \text { and } i_{R} d \lambda=0 .
$$

Note that in view of the Cartan formula we have $L_{R} \lambda=d(\lambda(R))+i_{R} d \lambda$, and hence the second condition can be restated as invariance of $\lambda$ under the flow of $R$.

Proof Suppose that there exists $\lambda$ which satisfies the above conditions. Set $\widetilde{\Theta}=\Theta+s \lambda$ and $\widetilde{\Omega}=d \widetilde{\Theta}$. Then for $\widetilde{\Omega}_{s}:=\left.\widetilde{\Omega}\right|_{V \times s}=\Omega+s d \lambda$ we have

$$
i_{R} \widetilde{\Omega}_{s}=i_{R} \Omega+s i_{R} d \lambda=0,
$$

and hence $(V,(\Theta))$ is stable. 
Conversely, suppose that a Hamiltonian structure $(V,(\Theta))$ is stable. Then according to the definition it has a symplectic extension $(V \times(-\varepsilon, \varepsilon), \widetilde{\Omega}=d \widetilde{\Theta})$ such that $i_{R} \widetilde{\Omega}_{s}=0$, where $\widetilde{\Omega}_{s}=\left.\widetilde{\Omega}\right|_{V \times s}$ and $R$ is a characteristic vector field for $\Omega$. Let us write $\widetilde{\Omega}$ as $\Omega+\eta_{s}+\lambda_{s} \wedge d s$, where $\lambda_{s}, \eta_{s}$ are families of $1-$ and 2 -forms on $V$ and $\eta_{0}=0$. We have

$$
0=d \widetilde{\Omega}=d \Omega+d \eta_{s}-\dot{\eta}_{s} \wedge d s+d \lambda_{s} \wedge d s=d \eta_{s}-\dot{\eta}_{s} \wedge d s+d \lambda_{s} \wedge d s,
$$

where $\dot{\eta}_{s}=\frac{d \eta_{s}}{d s}$. Hence, $d \lambda_{s}=\dot{\eta}_{s}$. On the other hand,

$$
0=i_{R} \widetilde{\Omega}_{s}=i_{R} \eta_{s}=0 .
$$

Differentiating with respect to $s$ we get $i_{R} \dot{\eta}_{s}=0$, and therefore, $i_{R} d \lambda_{s}=0$. Note that $\lambda_{0}(R)$ does nor vanish. Indeed along $V \times 0$ we have $\widetilde{\Omega}^{n+1}=\Omega^{n} \wedge \lambda_{0} \wedge d s$, and thus $0 \neq i_{R} \widetilde{\Omega}^{n+1}=\lambda_{0}(R) \Omega^{n} \wedge d s$. (Here $\operatorname{dim} V=2 n+1$.) Normalizing $R$ in such a way that $\lambda_{0}(R) \equiv 1$, we get that $\lambda_{0}(R)=1$ and $i_{R} d \lambda_{0}=0$, as required.

The structures characterized by existence of a 1 -form $\lambda$ satisfying the conditions of Proposition 4.1 appeared in [6], but were not identified there with stable hypersurfaces in symplectic manifolds which were first studied in [32].

The 1 -form $\lambda$ as in Proposition 4.1 is called a framing of the stable Hamiltonian structure $\mathcal{H}=(V,(\Theta))$. The hyperplane field $\xi=\{\lambda=0\}$, called a cut of $\mathcal{H}$, and the characteristic vector field $R$, called a Reeb field of the Hamiltonian structure, are uniquely determined from (44) by the framing $\lambda$. The triple $(V,(\Theta), \lambda)$ is called a framed Hamiltonian structure and is denoted by $\overrightarrow{\mathcal{H}}$. Here are examples of stable Hamiltonian structures taken from [6].

\section{Example 4.2}

1 Contact forms Let $\xi$ be a contact structure on $V$ and $\lambda$ a corresponding contact form. Then $(V,(\lambda), \lambda)$ is a framed stable Hamiltonian structure with cut $\xi$. In this case $R$ the usual Reeb field of the contact form $\lambda$ and $\Omega=d \lambda$.

2 Hamiltonian functions Let $(M, \omega=d \alpha)$ be an exact symplectic manifold and $H_{t}: M \rightarrow \mathbb{R}, t \in S^{1}=\mathbb{R} / \mathbb{Z}$, a 1 -periodic time-dependent Hamiltonian function. Write $\operatorname{sgrad} H_{t}$ for its Hamiltonian vector field. Put $V=M \times S^{1}$. Set $\Theta=-\alpha+H_{t} d t$ and $\lambda=d t$. Then $(V,(\Theta), \lambda)$ is a framed stable Hamiltonian structure. Its Reeb vector field is given by $R=\frac{\partial}{\partial t}+\operatorname{sgrad} H_{t}$ and its cut $\xi=\{\lambda=0\}$ is formed by tangent spaces to $M \times t, t \in S^{1}$.

3 Prequantization spaces Let $(M, \omega)$ be a symplectic manifold with the integral cohomology class $[\omega]$ of the symplectic form $\omega$. Consider the corresponding prequantization space, that is a principal $S^{1}$-bundle $p: V \rightarrow M$ with the first Chern class $[\omega]$. 
In this case the lift $\Omega:=p^{*} \omega$ of the symplectic form is exact: $\Omega=d \Theta$. Then $(V,(\Theta))$ is a stable Hamiltonian structure. Indeed, one can choose any $S^{1}$-connection form $\lambda$ as its framing. The corresponding Reeb vector field $R$ is the infinitesimal generator of the $S^{1}$-action, and the cut $\xi$ is the horizontal distribution of the connection.

\subsection{Special Hamiltonian structures}

As it is stated above, the goal of Section 4 is to develop the Floer homology theory for Hamiltonian structures. To avoid unnecessary technicalities we will restrict the theory to a special class of stable Hamiltonian structures which will be sufficient for all the applications considered in this paper. In Section 4.11 we briefly discuss possible generalizations of the theory.

Let $\left(M^{2 n}, \omega, L\right)$ be a Liouville manifold with the Liouville form $\alpha$. We will work on the manifold $V=M \times S^{1}$, write $t(\bmod 1)$ for the coordinate on $S^{1}=\mathbb{R} / \mathbb{Z}$, and orient $V$ by the volume form $(-\omega)^{n} \wedge d t$.

Let us denote by $F_{\mathrm{C}, \mathrm{K}}$ the set of all 1 -forms on $V$ which coincide with $\mathrm{C} d t-\mathrm{K} \alpha$ $(\mathrm{C}, \mathrm{K} \in \mathbb{R})$ outside a compact subset of $V$. We identify the set $F:=\bigcup_{\mathrm{C}, \mathrm{K} \in \mathbb{R}} F_{\mathrm{C}, \mathrm{K}}$ with $\mathbb{R} \times \mathbb{R} \times F_{0,0}$ and equip it with the product topology, where $F_{0,0}$ is considered with the strong Whitney topology. It induces a topology on the set of equivalence classes of $1-$ forms.

A Hamiltonian structure $\mathcal{H}=(V,(\Theta))$ on $V$ is called special if $\Theta \in F_{\mathrm{C}, \mathrm{K}}$, where $\mathrm{C}$ and $\mathrm{K}$ are positive constants which are called the structure constants of $\mathcal{H}$, and the following Axioms 1-3 are satisfied.

Axiom 1 The first Chern class of the symplectic vector bundle $(T V / \ell, \Omega=d(\Theta))$, where $\ell$ is the characteristic line field of $\mathcal{H}$, vanishes on any 2-cycle represented by a mapping $\mathbb{T}^{2} \rightarrow V$.

Recall that the characteristic foliation $\mathcal{T}$ of $\mathcal{H}$ is equipped with an orientation. The action of a periodic orbit $\gamma$ of $\mathcal{T}$ is defined by

$$
\mathcal{A}(\gamma)=\int_{\gamma} \Theta .
$$

By Stokes' formula, the action does not depend on the specific choice of a 1-form $\Theta$ representing $(\Theta)$. In view of the conditions "at infinity" imposed on $\Theta$, the trajectories of $\mathcal{T}$ outside a sufficiently large compact subset of $V$ are circles \{point\} $\times S^{1}$, and their action equals to the structure constant $C$. 
Axiom 2 The characteristic foliation $\mathcal{T}$ has no contractible closed orbits of action $\leq \mathrm{C}$.

Axiom 3 There exist numbers $\mu \geq 0, \mathrm{P}>0, \mathrm{Q} \geq 0$ and a 1 -form $\lambda \in F_{\mathrm{P}, \mathrm{Q}}$ such that $\lambda-\mu \Theta$ is a closed $1-$ form representing cohomology class $(\mathrm{P}-\mu \mathrm{C})[d t]$ and $\lambda$ is positive on the characteristic foliation $\mathcal{T}$ with respect to its natural orientation.

Note that in view of Axiom 3 we have $d \lambda=\mu \Omega$, which implies stability of $\mathcal{H}$. In particular, $\lambda$ is a framing of the Hamiltonian structure $\mathcal{H}$. Such a framing is called special. Special framings form a convex cone. The constant $\mu$ is called the parameter, and the numbers $P$ and $Q$ the structure constants of the framing. Note that $Q=\mu \mathrm{K}$. Clearly, the cut $\xi$ is a contact structure on $V$ if $\mu>0$, and $\xi$ integrates to a foliation defined by a closed 1 -form if $\mu=0$. Sometimes, in order to emphasize the dependence of the introduced objects on $\mathcal{H}$ we will write $(\Theta)_{\mathcal{H}}, \Omega_{\mathcal{H}}, \mathcal{T}_{\mathcal{H}}, \mathcal{A}_{\mathcal{H}}$ etc.

Consider a special Hamiltonian structure $(V,(\Theta))$ with a special framing $\lambda$. We start with the following useful period-action equation, which is an immediate consequence of the definitions. Let $\gamma$ be a $T$-periodic closed orbit of the Reeb vector field $R$. Then

$$
T=\mu \mathcal{A}(\gamma)+(\mathrm{P}-\mu \mathrm{C})\langle[d t],[\gamma]\rangle
$$

Proposition 4.3 Let $(\Theta)$ be a special Hamiltonian structure on $V$. Then every 1-form $\Theta^{\prime}$ in a sufficiently small neighborhood of $\Theta$ defines a special Hamiltonian structure. Moreover, if $\lambda$ is a special framing of $\Theta$ then there exists a special framing $\lambda^{\prime}$ of $\Theta^{\prime}$ which is sufficiently close to $\lambda$.

Proof If $\Theta^{\prime}$ is sufficiently close to $\Theta$ then Axiom 1 for $\Theta^{\prime}$ is fulfilled automatically. To check Axiom 3, note that the 1-form

$$
\lambda^{\prime}=\lambda+\mu\left(\Theta^{\prime}-\Theta\right)
$$

is a framing of $\left(\Theta^{\prime}\right)$ with the same parameter $\mu$. It remains to verify Axiom 2. Note that if $\mu=0$ then in view of equation (46) the characteristic foliation $\mathcal{T}$ of $\left(\Theta^{\prime}\right)$ cannot have contractible closed orbits. Let us assume that $\mu>0$ and suppose on the contrary that there exists a sequence of $1-$ forms $\Theta_{i} \rightarrow \Theta$ whose characteristic foliations admit contractible closed orbits of action $\leq \mathrm{C}_{i}$, where $\mathrm{C}_{i}$ is the structure constant of $\Theta_{i}$. Applying again (46) we see that the periods of $\gamma_{i}$ are bounded, and thus these orbits converge to a contractible closed orbit of $(\Theta)$ of action $\leq \mathrm{C}$. This contradicts to Axiom 2 for $\Theta$. 
We will be mostly dealing in this paper with special Hamiltonian structures, and hence, if not otherwise noted,

$$
\begin{aligned}
& \text { all considered Hamiltonian structures and } \\
& \text { their framings will be assumed special. }
\end{aligned}
$$

\subsection{Basic examples}

The following two basic examples of special Hamiltonian structures which will appear below (see [6]) are specifications of Examples 4.2.1 and 4.2.2.

Example 4.4 (Contact forms) Let $F: V \rightarrow \mathbb{R}$ be a positive function which is equal to a constant $\mathrm{C}$ outside a compact set. Consider a contact form $\lambda:=F(d t-\alpha)$ on $V$ whose Reeb vector field has no contractible closed orbits of period $\leq \mathrm{C}$. We call such a form admissible. Then $(\lambda)$ is a Hamiltonian structure on $V$. The same 1-form $\lambda$ serves as its framing, and so the framing parameter $\mu$ equals 1 . All the structure constants are equal to $\mathrm{C}$. The cut $\xi=\operatorname{Ker}(\lambda)$ is a contact structure on $V$, and the Reeb vector field is the usual Reeb field of $\lambda$. One can easily check that the symplectic subbundles $(\xi, \Omega=d \lambda)$ and $(T M,-\omega)$ of $T V$ are homotopic, and thus have the same first Chern classes (see Section 4.13 for more details). Hence, Axiom 1 is equivalent to the requirement that $c_{1}(T M)$ vanishes on any cycle represented by a mapping $\mathbb{T}^{2} \rightarrow M$. Verification of other axioms is straightforward. We denote the obtained framed Hamiltonian structure by $\overrightarrow{\mathcal{H}}_{\lambda}$, as it is fully determined by the contact form $\lambda$ on $V$.

Example 4.5 (Hamiltonian functions) Let $F: V=M \times S^{1} \rightarrow \mathbb{R}$ be a time-dependent 1-periodic Hamiltonian function on $M$ which is equal to a positive constant $\mathrm{C}$ outside a compact set. Let us introduce a Hamiltonian structure $(F d t-\alpha)$ on $V$. The framing is chosen as $\lambda:=d t$, and hence the framing parameter $\mu$ vanishes. Thus the cut $\xi$ is the tangent bundle to the fibers $M \times\{$ point $\}$, and the Reeb vector field is $R=\frac{\partial}{\partial t}+\operatorname{sgrad} F$. As in the previous example it is straightforward that all axioms are satisfied provided that $c_{1}(T M)$ vanishes on any cycle represented by a mapping $\mathbb{T}^{2} \rightarrow M$. This framed Hamiltonian structure is fully determined by the function $F$ and will be denoted by $\overrightarrow{\mathcal{H}}_{F}$.

\subsection{Periodic orbits of the characteristic foliation}

Let $\mathcal{T}$ be the oriented characteristic foliation of a Hamiltonian structure $\mathcal{H}=(\Theta)$. Write $C$ for the structure constant. Denote by $\mathcal{S}$ the set of free homotopy classes of loops $S^{1} \rightarrow V$ which project to the class [point $\times S^{1}$ ] under the natural projection 
$V=M \times S^{1} \rightarrow S^{1}$. We will focus on those orbits of $\mathcal{T}$ with action $<\mathrm{C}$ which represent classes from $\mathcal{S}$, and denote the set of such orbits by $\mathcal{P}$.

Definition 4.6 A Hamiltonian structure is called regular if all periodic orbits from $\mathcal{P}$ are non-degenerate, that is their linearized Poincaré return maps do not contain 1 in their spectra.

Proposition 4.7 Regular Hamiltonian structures form a Baire set in the space of all Hamiltonian structures.

Outline of the proof A little nuance here is that the "flat" orbits at infinity, whose action equals $\mathrm{C}$, are certainly degenerate. One goes round this difficulty as follows. Choose a starshaped hypersurface $P \subset M$ such that $M=\operatorname{Core}(M) \bigsqcup S P$, and introduce coordinates $(x, u)$ on $S P=P \times \mathbb{R}_{+}$. In these coordinates $\alpha=u \beta$ where $\beta$ is the contact form on $P$ defined by the restriction of $\alpha$. Denote by $R_{\beta}$ the Reeb field of $\beta$ and by $T$ the minimal period of a closed orbit of $R_{\beta}$.

Let $\mathcal{H}=(\Theta)$ be any Hamiltonian structure with the structure constants $\mathrm{C}$ and $\mathrm{K}$. We can assume without loss of generality that $\mathrm{K}=1$ and $\Theta=\mathrm{C} d t-u \beta$ for $u \geq 1 / 2$. We claim that there exists an arbitrarily small perturbation $\Theta_{1}$ of $\Theta$ such that $\Theta_{1}=\Theta$ for $u \geq 2$ and the region $A:=\{1 \leq u<2\}$ does not contain closed orbits of the characteristic foliation of $\left(\Theta_{1}\right)$ representing classes from $\mathcal{S}$. Indeed, write $\Theta_{1}=G(u) \Theta$, where $G(u) \equiv 1$ for $u \geq 2$. Put $H(u)=C G^{\prime} /(G u)^{\prime}$. Assume that for $u \in[1,2)$ the function $G$ is strictly increasing and $C^{1}$-close to the constant function $u \equiv 1$. Then the characteristic foliation $\mathcal{T}_{1}$ of $\left(\Theta_{1}\right)$ on $A$ is generated by the field $\frac{\partial}{\partial t}+H(u) R_{\beta}$ with $0<H(u)<1 / T$. Thus $\mathcal{T}_{1}$ has no closed orbits in $A$ representing classes from $\mathcal{S}$. Now one can extend $\Theta_{1}$ to the rest of $V$ to get the desired perturbation.

It remains to perturb $\Theta_{1}$, keeping it fixed on the set $\{u>3 / 2\}$, in such a way that all closed orbits of the characteristic foliation outside this set will be non-degenerate. This can be done by a standard adaptation of the Kupka-Smale type argument, see eg [40].

Remark 4.8 Period-action equation (46) guarantees that the periods of all orbits from $\mathcal{P}$ are bounded. Indeed, given an orbit from $\mathcal{P}$ of period $T$ we have

$$
T \leq \mu \mathrm{C}+(\mathrm{P}-\mu \mathrm{C}) \cdot 1=\mathrm{P} .
$$

Thus for a regular Hamiltonian structure and for any $\varepsilon>0$ the set $\{\gamma \in \mathcal{P} \mid \mathcal{A}(\gamma) \leq \mathrm{C}-\varepsilon\}$ is finite. 
Consider the functional $\mathcal{A}_{\mathcal{H}}(\gamma)=\int_{\gamma} \Theta$ defined on the space of smooth loops $S^{1} \rightarrow V$ representing classes from $\mathcal{S}$. Define the action spectrum spec $\mathcal{H}$ of a Hamiltonian structure $\mathcal{H}$ as the set of values of $\mathcal{A}_{\mathcal{H}}$ on orbits from $\mathcal{P}$. Note that this set can be interpreted as the set of critical values of $\mathcal{A}_{\mathcal{H}}$ lying in the interval $(-\infty ; C)$. Indeed, the critical points of $\mathcal{A}_{\mathcal{H}}$ correspond to closed orbits of the characteristic foliation parameterized in an arbitrary way by the circle $S^{1}$. Using period-action equation (46) one can show (by a lengthy, but quite straightforward modification of the standard argument, cf [32]) that even for a non-regular Hamiltonian structure $\mathcal{H}$ the set spec $\mathcal{H} \cup$ $\{C\}$ is a closed nowhere dense subset of $\mathbb{R}$. The points from $(0, C) \backslash \operatorname{spec} \mathcal{H}$ are called non-critical values of $\mathcal{A}_{\mathcal{H}}$. For two non-critical values $a, b$ we denote by $\mathcal{P}^{(a ; b)}$ the subset of $\mathcal{P}$ which consists of periodic orbits $\gamma$ with $a<\mathcal{A}_{\mathcal{H}}(\gamma)<b$.

4.4.1 The Conley-Zehnder index and the grading Recall that the Conley-Zehnder index is an integer number associated to a path of symplectic matrices $A=A(t), t \in$ [0,1], where $A(0)=1$ and $A(t)$ does not contain 1 in its spectrum, see eg [41]. It is denoted by $\mathrm{CZ}(A)$. Different authors use different conventions on the sign and normalization (up to an additive constant) of the Conley-Zehnder index. Our convention is as follows:

- If a path $A$ of symplectic matrices is generated by a sufficiently small quadratic Hamiltonian $F$ on $\mathbb{R}^{2 n}=\mathbb{C}^{n}$ then the Conley-Zehnder index of $A$ equals the Morse index of $F$, that is the number of negative squares;

- Given a path $\gamma_{1}$ and a loop $\gamma_{2}$ of symplectic matrices, the Conley-Zehnder index of their concatenation satisfies

$$
\mathrm{CZ}\left(\gamma_{1} \sharp \gamma_{2}\right)=\mathrm{CZ}\left(\gamma_{1}\right)-\operatorname{Maslov}\left(\gamma_{2}\right)
$$

(note the minus sign!), where Maslov stands for the Maslov index of a loop;

- The Maslov index is normalized by

$$
\operatorname{Maslov}\left(\left\{e^{2 \pi i t}\right\}_{t \in[0,1]}\right)=+2 .
$$

The Conley-Zehnder index plays a crucial role in the definition of a grading on the space $C=\operatorname{Span}_{\mathbb{Z}_{2}}(\mathcal{P})$ which in turn is a basic ingredient in the definition of generalized Floer homology. Let us discuss this grading in more details. ${ }^{4}$ First of all, given a free homotopy class $e \in \mathcal{S}$ let us denote by $\mathcal{P}_{e}$ the set of all orbits from $\mathcal{P}$ which represent $e$. Set $C_{e}=\operatorname{Span}_{\mathbb{Z}_{2}}\left(\mathcal{P}_{e}\right)$. Then we have a decomposition $C=\oplus_{e \in \mathcal{S}} C_{e}$.

Fix a class $e \in \mathcal{S}$, and take any loop $\phi_{0}: S^{1} \rightarrow V$ representing $e$. Pick a symplectic trivialization $g_{0}: \phi_{0}^{*}(T V / \ell) \rightarrow \mathbb{R}^{2 n} \times S^{1}$ of the symplectic vector bundle

\footnotetext{
${ }^{4}$ This material is essentially known to the experts.
} 
$\left(\phi_{0}^{*}(T V / \ell), d(\Theta)\right)$ over $S^{1}$. Given any other loop $\phi_{1}: S^{1} \rightarrow V$ representing $e$, choose a homotopy $\Phi: S^{1} \times[0 ; 1]$ between $\phi_{0}$ and $\phi_{1}$ with $\left.\Phi\right|_{S^{1} \times\{i\}}=\phi_{i}, i \in\{0,1\}$. Extend $g_{0}$ to a symplectic trivialization of $\Phi^{*}(T V / \ell)$ and denote by $g_{1}$ its restriction to $S^{1} \times\{1\}$. We get a symplectic trivialization of the bundle $\left(\phi_{1}^{*}(T V / \ell), d(\Theta)\right)$. Axiom 1 in the definition of a special Hamiltonian structure guarantees that $g_{1}$ does not depend on the choice of the homotopy $\Phi$ up to multiplication by a contractible loop of symplectic matrices. We say that trivializations $g_{0}$ and $g_{1}$ are equivalent, and call the equivalence class a coherent trivialization of the bundle $T V / \ell$ in the class $e$. The set of coherent trivializations in the class $e$ is denoted by $\rrbracket_{e}$. Importantly, this set carries a natural action of $\mathbb{Z}$. Indeed, consider a coherent trivialization $j \in \mathbb{J}_{e}$ as above induced by a pair $(\phi, g)$, where $\phi: S^{1} \rightarrow V$ is a loop and $g: \phi^{*}(T V / \ell) \rightarrow \mathbb{R}^{2 n} \times S^{1}$ is a symplectic trivialization. Take any loop $A(t)$ of symplectic matrices with the Maslov index $2 k, k \in \mathbb{Z}$. Define a map

$$
\bar{A}: \mathbb{R}^{2 n} \times S^{1} \rightarrow \mathbb{R}^{2 n} \times S^{1}
$$

by $\bar{A}(x, t)=(A(t) x, t)$. Consider a new trivialization $g^{\prime}=\bar{A} \circ g$ of $\phi^{*}(T V / \ell)$, and denote by $j^{\prime}$ the induced coherent trivialization. By definition, $j^{\prime}$ is the result of the action of $k \in \mathbb{Z}$ on $j$, which is denoted by $j^{\prime}=j+k$. One can easily check that this $\mathbb{Z}$-action on $\mathbb{J}_{e}$ is well defined, transitive and free.

Choose any framing of a regular Hamiltonian structure $(V,(\Theta))$. Denote by $R^{t}: V \rightarrow V$ the flow of the corresponding Reeb vector field. The linearization $R_{*}^{t}$ of this flow acts by symplectic automorphisms of the bundle $(T V / \ell, d(\Theta))$. Let $p(t)$ be a closed orbit of $R^{t}$ from $\mathcal{P}_{e}$ with period $T$. Put $\tilde{p}(t)=p(T t)$. Every coherent trivialization $j \in \rrbracket_{e}$ gives rise to a symplectic trivialization

$$
g: \tilde{p}^{*}(T V / \ell) \rightarrow \mathbb{R}^{2 n} \times S^{1} .
$$

Denote by $g_{t}$ its restriction to the fiber over $t \in S^{1}$. Define the Conley-Zehnder index $\mathrm{CZ}(p, j)$ of the orbit $p \in \mathcal{P}_{e}$ with respect to the coherent trivialization $j$ as the Conley-Zehnder index of the loop $g_{t} R_{*}^{t T} g_{0}^{-1}$ of symplectic matrices. One readily checks that this definition does not depend on the choice of a special framing. Taking into account our convention on the Conley-Zehnder index and the definition of the $\mathbb{Z}$-action on $\rrbracket_{e}$ we have

$$
\mathrm{CZ}(p, j+k)=\mathrm{CZ}(p, j)-2 k \text { for all } k \in \mathbb{Z} .
$$

As an immediate consequence we get that for each pair of orbits $p, p^{\prime} \in \mathcal{P}_{e}$ the difference $\mathrm{CZ}(p, j)-\mathrm{CZ}\left(p^{\prime}, j\right)$ does not depend on the choice of a coherent trivialization $j \in \mathbb{J}_{e}$. Thus we often denote it by $\mathrm{CZ}(p)-\mathrm{CZ}\left(p^{\prime}\right)$ even though each term in this expression depends on $j$. We conclude that the space $C_{e}$ is equipped with $a \mathbb{Z}$-grading up to an 
additive shift. Formula (47) yields, however, that the even and the odd parts of $C_{e}$ are well defined.

Instead of speaking about $a \mathbb{Z}$-grading up to an additive shift we prefer to adopt the following more concrete viewpoint. Let us call a $\mathbb{Z}$-set any set equipped with a free transitive $\mathbb{Z}$-action. Given such a set, say $Y$, one introduces the notions of a $Y$-graded vector space and a $Y$-graded chain complex. The homology groups of a $Y$-graded chain complex are naturally $Y$-graded.

Introduce a structure of a $\mathbb{Z}$-set on

$$
\rrbracket_{e}:=\rrbracket_{e} \times\{0 ; 1\}
$$

as follows: put $(j, 0)+1=(j, 1)$ and $(j, 1)+1=(j+1,0)$. For an element $p \in \mathcal{P}_{e}$ define its degree $\operatorname{deg}(p) \in \mathbb{\square}_{e}$ as $(j, 0)$ if $\mathrm{CZ}(p, j)=0$ and as $(j, 1)$ if $\mathrm{CZ}(p, j)=1$. One can easily check that the degree is well defined and

$$
\operatorname{deg}\left(p^{\prime}\right)=\operatorname{deg}(p)+\left(\mathrm{CZ}\left(p^{\prime}\right)-\mathrm{CZ}(p)\right)
$$

for all $p, p^{\prime} \in \mathcal{P}$. Thus we endowed the space $C_{e}$ with $\rrbracket_{e}$-grading. Put

$$
\llbracket=\left\{(e, i): e \in \mathcal{S}, i \in \mathbb{\natural}_{e}\right\} .
$$

Then

$$
C=\oplus_{(e, i) \in \llbracket} C_{e, i}
$$

Definition 4.9 The set $\llbracket$ (respectively, $\rrbracket_{e}$ ) is called the grading set of the Hamiltonian structure $\mathcal{H}$ (respectively, in the class $e$ ).

In Section 4.7 below we shall define generalized Floer homology of a Hamiltonian structure $\mathcal{H}$ as the homology of $(C, d)$ where $d: C \rightarrow C$ is a differential which maps $C_{e, i} \rightarrow C_{e, i-1}$. Hence the homology will inherit the same -grading.

We write $\rrbracket_{e}(\mathcal{H}), \rrbracket_{e}(\mathcal{H})$ and $\square(\mathcal{H})$ whenever we wish to emphasize dependence of the set of coherent trivializations and the grading sets on the Hamiltonian structure $\mathcal{H}$.

Remark 4.10 In the case when $e=\left[\right.$ point $\left.\times S^{1}\right]$ the set $\rrbracket_{e}$ has a distinguished element. Namely, take any point $x \in M$ outside a sufficiently large compact subset of $M$ where $\Theta=\mathrm{C} d t-\mathrm{K} \alpha$. Consider the loop $\phi(t)=(x, t)$ and notice that $\left(\phi^{*} T V / \ell, d \Theta\right)$ can be naturally identified with $\left(T_{x} M \times S^{1},-\mathrm{K} d \alpha\right)$. Denote by $j_{0} \in \mathbb{J}_{e}$ the corresponding coherent trivialization, and put $i_{0}=\left(j_{0}, 0\right)$. Identify $\mathbb{Z}$ with $\rrbracket_{e}$ via the map $k \rightarrow i_{0}+k$. With this identification, $\operatorname{deg}(p)=\operatorname{CZ}\left(p, j_{0}\right)$ for every $p \in \mathcal{P}_{\boldsymbol{e}}$. Thus we have a canonical $\mathbb{Z}$-grading of $C_{e}$. This remark is especially useful when $M$ is simply connected, for instance $M=\mathbb{R}^{2 n}$. Let us mention also that if we wish to restrict 
ourselves to periodic orbits from the class [point $\times S^{1}$ ] only, we can relax Axiom 1 of special Hamiltonian structures and require that the first Chern class of $(T V / \ell, d(\Theta))$ vanishes on all spherical 2-cycles.

Remark 4.11 Another possibility to introduce a natural $\mathbb{Z}$-grading on the spaces $C_{e}$ for all $e$ appears in the case when the bundle $(T V / \ell, d(\Theta))$ is equipped with a Lagrangian subbundle, say, $L$. In this case we choose any almost complex structure $J$ on $T V / \ell$ compatible with $d(\Theta)$. Given any loop $\phi: S^{1} \rightarrow V$ representing the class $e$, we trivialize $\phi^{*}(T V / \ell)$ by choosing the homotopically unique $J$-unitary frame in $\phi^{*} L \subset \phi^{*}(T V / \ell)$. This procedure is applied, for instance, when $(M, \omega)$ is the cotangent bundle of a closed manifold $X$ equipped with the standard symplectic form, and the Hamiltonian structure $(V,(\Theta))$ is associated to a time-dependent Hamiltonian function on $M$ as in Example 4.5 above. Here the bundle $(T V / \ell, d(\Theta))$ can be identified with $\left(T T^{*} X,-\omega\right)$ and the Lagrangian subbundle $L \subset T T^{*} X$ is simply formed by the tangent spaces to the fibers of $T^{*} X \rightarrow X$.

\subsection{Adjusted almost complex structures}

Next, we introduce a class of almost complex structures compatible with a framed Hamiltonian structure $\overrightarrow{\mathcal{H}}=((\Theta), \lambda)$. These almost complex structures are defined on a manifold $\bar{V}=V \times \mathbb{R}$.

Take any starshaped hypersurface $P \subset M$, and denote by $\eta=\operatorname{Ker}\left(\left.\alpha\right|_{T P}\right)$ the contact structure on $P$. In view of the canonical splittings $M \backslash \operatorname{Core}(M)=P \times \mathbb{R}_{+}$and $\bar{V}=M \times S^{1} \times \mathbb{R}$ we will consider the cut of $\overrightarrow{\mathcal{H}}$, denoted by $\xi$, and the contact plane field $\eta$ on $P$ as subbundles of $T \bar{V}$.

The group $\mathbb{R}$ acts on $\bar{V}$ by translations $(z, s) \mapsto(z, s+c)$ where $s, c \in \mathbb{R}$ and $z \in V$.

Definition 4.12 An $\mathbb{R}$-invariant almost complex structure $J$ on $\bar{V}$ is called adjusted to the framed Hamiltonian structure $\overrightarrow{\mathcal{H}}$ if

- the cut $\xi$ of $\overrightarrow{\mathcal{H}}$ is $J$-invariant, and $\left.d(\Theta)\right|_{\xi}$ is compatible with $J$;

- $J \frac{\partial}{\partial s}=R$, where $R$ is the characteristic (Reeb) vector field;

- the contact plane field $\eta$ is $J$-invariant outside $Z \times \mathbb{R}$ where $Z \subset M$ is a sufficiently large compact subset.

An important feature of the almost complex manifold $(\bar{V}, J)$ with the adjusted $J$ is that it carries a natural foliation by $J$-holomorphic curves. It has the form $\mathcal{T}_{J}=\mathcal{T} \times \mathbb{R}$ where $\mathcal{T}$ is the characteristic foliation of $V$ generated by $R$. We call $\mathcal{T}_{J}$ the characteristic $J$-holomorphic foliation of $(\bar{V}, J)$. 
Remark 4.13 Suppose that $J$ is adjusted to a framed Hamiltonian structure $(\mathcal{H}, \lambda)$. Given $c>0$ let us define a new almost complex structure $J_{c}$ by setting $\left.J_{c}\right|_{\xi}=\left.J\right|_{\xi}$ and $J \frac{\partial}{\partial s}=\frac{1}{c} R$. Then $J_{c}$ is adjusted to the framed Hamiltonian structure $(\mathcal{H}, c \lambda)$, and the map

$$
(x, s) \mapsto(x, c s)
$$

is a biholomorphism

$$
(\bar{V}=V \times \mathbb{R}, J) \rightarrow\left(\bar{V}, J_{c}\right) .
$$

The next lemma will allow us to feel comfortable with pseudo-holomorphic curves in $(\bar{V}, J)$. Recall that a (co-)oriented hypersurface, say $\Sigma$, in an almost complex manifold $(W, J)$ is called weakly $J$-convex if there exists a $1-$ form $\sigma$ on $\Sigma$ such that

$$
T_{\mathbb{C}} \Sigma=\operatorname{Ker} \sigma,
$$

$d \sigma(v, J v) \geq 0$ for $v \in T_{\mathbb{C}} \Sigma$, and the orientation of $T_{\mathbb{C}} \Sigma$ determined by the orientation of $\Sigma$ and its co-orientation by $\sigma$ coincides with its complex orientation.

Write $z \mapsto c * z, c \in \mathbb{R}_{+}, z \in M$ for the natural $\mathbb{R}_{+}$-action on $M$ associated to the Liouville vector field. Consider a hypersurface

$$
\Sigma_{u}=(u * P) \times S^{1} \times \mathbb{R} \subset \bar{V} .
$$

Lemma 4.14 For all sufficiently large $u$ the hypersurface $\Sigma_{u}$ is weakly $J$-convex in $(\bar{V}, J)$.

Proof Suppose that $u$ is large enough. Then

$$
\Theta=\mathrm{C} d t-\mathrm{K} \alpha, \xi=\operatorname{Ker}(\mathrm{P} d t-\mathrm{Q} \alpha), R=\frac{1}{\mathrm{P}} \frac{\partial}{\partial t},
$$

where $C, K, P, Q$ are the structure constants of $\overrightarrow{\mathcal{H}}$. By definition,

$$
J \frac{\partial}{\partial s}=R=\frac{1}{\mathrm{P}} \frac{\partial}{\partial t},
$$

and hence the plane $\zeta:=\operatorname{Span}\left(\frac{\partial}{\partial s}, \frac{\partial}{\partial t}\right)$ is $J$-invariant. Furthermore, $\eta$ is also $J-$ invariant. Thus the hyperplane

$$
H_{u}:=\eta \oplus \zeta \subset T \Sigma_{u}
$$

is the complex tangent space to $\Sigma_{u}$ :

$$
H_{u}=T_{\mathbb{C}} \Sigma_{u}
$$


Note that $H_{u}=\operatorname{Ker}\left(\left.\alpha\right|_{T \Sigma_{u}}\right)$. Observe that $\eta$ is contained in $\xi$, since both $d t$ and $\alpha$ vanish on $\eta$. The $2-$ form $d \alpha$ coincides with the restriction of $-\mathrm{K}^{-1} d \Theta$ to $\Sigma_{u}$. Therefore, since $\left.d \Theta\right|_{\xi}$ is compatible with $J$, we obtain that $-d \alpha$ restricted to $\eta$ is also compatible with $J$. Finally, $d \alpha$ vanishes on $\zeta$. We conclude that the hyperplane $T_{\mathbb{C}} \Sigma_{u} \subset T \Sigma_{u}$, which coincides with $H_{u}$, and the 1-form $-\alpha$ on $\Sigma_{u}$ satisfy the definition of weak $J$-convexity given above. We leave it to the reader to verify the orientation condition.

\section{6 $J$-holomorphic cylinders in $\bar{V}$}

Let $\overrightarrow{\mathcal{H}}$ be a framed Hamiltonian structure. Take an adjusted almost complex structure $J$ on $\bar{V}:=V \times \mathbb{R}$. Write $\Upsilon=\mathbb{R} \times S^{1}$ for the standard cylinder with coordinates $\sigma \in \mathbb{R}$ and $\tau \in S^{1}$. We endow $\Upsilon$ with the standard complex structure by introducing the complex coordinate $z=\sigma+i \tau$. Given two orbits $\gamma_{ \pm} \in \mathcal{P}$, we consider $J$-holomorphic cylinders

$$
F=(f, \varphi): \Upsilon \rightarrow V \times \mathbb{R}, \quad \frac{\partial F}{\partial \sigma}+J \frac{\partial F}{\partial \tau}=0
$$

with asymptotic boundary conditions

$$
\begin{aligned}
& \varphi(\sigma, \tau) \underset{\sigma \rightarrow \pm \infty}{\rightarrow}= \pm \infty, \\
& f(\sigma, \tau) \underset{\sigma \rightarrow \pm \infty}{\rightarrow}=\gamma_{ \pm}\left(T_{ \pm} \tau\right),
\end{aligned}
$$

where the orbits $\gamma_{ \pm}$are parameterized by the Reeb field $R$, and $T_{ \pm}$are their periods. Denote by $\hat{\mathcal{M}}\left(\gamma_{+}, \gamma_{-}\right)$the set of solutions of this system. The group $\Gamma:=\mathbb{R} \times \mathbb{R} \times S^{1}$ acts on $\hat{\mathcal{M}}\left(\gamma_{+}, \gamma_{-}\right)$as follows:

$$
(a, b, \vartheta) * F=G \text {, where } G(\sigma, \tau)=(F(\sigma+a, \tau+\vartheta), \varphi(\sigma+a, \tau+\vartheta)+b) .
$$

The action is free provided $\gamma_{-} \neq \gamma_{+}$. This is a straightforward consequence of the fact that the orbits $\gamma_{-}$and $\gamma_{+}$represent a homotopy class from $\mathcal{S}$, and therefore are simple (ie not multiply covered). Let us mention also that if $\gamma=\gamma_{-}=\gamma_{+}$then the solution set $\hat{\mathcal{M}}(\gamma, \gamma)$ consists of maps of the form

$$
(\sigma, \tau) \mapsto(\gamma(T(\tau+b)), \sigma+a),
$$

which represent the leaf of the $J$-holomorphic characteristic foliation of $\bar{V}$ corresponding to $\gamma \times \mathbb{R}$.

Definition 4.15 An adjusted almost complex structure $J$ is called regular if at every $J$-holomorphic cylinder which satisfies (49) the linearized $\bar{\partial}$-operator is surjective. 
Proposition 4.16 Regular almost complex structures are generic, that is they form a Baire set with respect to a natural topology.

The proof of this statement is scattered in the literature for various classes of framed Hamiltonian structures (see eg $[31 ; 14 ; 5]$ ). The cornerstone of the proof is the following lemma, which we prove in detail in Appendix A. The reader is referred to the above-cited papers for the rest of the argument. Consider a $J$-holomorphic map $F=(f, \varphi): \Upsilon \rightarrow \bar{V}$ which converges to two distinct simple periodic orbits $\gamma_{ \pm}$as $\sigma \rightarrow \pm \infty$. A point $z \in \Upsilon$ is called an injectivity point of $F$ if $d_{z} f$ is injective and $f^{-1}(f(z))=z$.

Lemma 4.17 There is an open dense set in $\Upsilon$ consisting of the points of injectivity.

The proof is given in Appendix A.

Assume that $J$ is a regular almost complex structure. Then for a pair of distinct periodic orbits $\gamma_{+}, \gamma_{-} \in \mathcal{P}$ the moduli space

$$
\mathcal{M}\left(\gamma_{+}, \gamma_{-}\right)=\widehat{\mathcal{M}}\left(\gamma_{+}, \gamma_{-}\right) / \Gamma
$$

is a smooth manifold of dimension $\left(\mathrm{CZ}\left(\gamma_{+}\right)-\mathrm{CZ}\left(\gamma_{-}\right)\right)-1$. In particular, when $\mathrm{CZ}\left(\gamma_{+}\right)-\mathrm{CZ}\left(\gamma_{-}\right)=1$ the corresponding moduli space is zero-dimensional. Moreover, this set is compact, and hence consists of a finite number of points. The compactness can be proved along the following lines. First, by using Lemma 4.14 above one shows that $J$-holomorphic cylinders in question project to a compact subset of $V$. Then, as it was proved in [6], the compactness follows from the absence of contractible closed orbits of the characteristic vector field of action $\leq \mathrm{C}$.

\subsection{Floer homology of framed Hamiltonian structures}

Let $\overrightarrow{\mathcal{H}}$ be a regular framed Hamiltonian structure with an adjusted regular almost complex structure $J$. The Floer complex can be defined in a usual way. Assuming $\mathrm{CZ}\left(\gamma_{+}\right)-\mathrm{CZ}\left(\gamma_{-}\right)=1$ we denote by $v\left(\gamma_{+}, \gamma_{-}\right)$the $\bmod 2$ number of elements of the finite set $\mathcal{M}\left(\gamma_{+}, \gamma_{-}\right)$and consider the $\mathbb{Z}_{2}$-space $C$ generated by the orbits from $\mathcal{P}$. The boundary operator $d: C \rightarrow C$ is defined by

$$
d \gamma_{+}=\sum_{\substack{\gamma_{-} \in \mathcal{P} \\ \mathrm{CZ}\left(\gamma_{+}\right)-\mathrm{CZ}\left(\gamma_{-}\right)=1}} v\left(\gamma_{+}, \gamma_{-}\right) \gamma_{-} .
$$

The space $C$ is graded by elements of the grading set $\square(\mathcal{H})$, see Definition 4.9. The differential $d$ maps $C_{e, i}$ to $C_{e, i-1}$ for every $(e, i) \in \mathbb{\square}$. 
The proof of the following theorem repeats the standard arguments in the Floer homology theory; one have to take into account Lemma 4.14 and compactness results from [6].

Theorem 4.18 $d^{2}=0$.

Note that the boundary operator $d$ preserves the filtration defined by the action functional $\mathcal{A}_{\mathcal{H}}$. Hence, for any $a<b<\mathrm{C}$ we get a chain complex $\left(C^{(a, b)}(\overrightarrow{\mathcal{H}}, J), d\right)$, where

$$
C^{(a, b)}=(C \cap\{\mathcal{A}(\gamma)<b\}) /(C \cap\{\mathcal{A}(\gamma) \leq a\}) .
$$

Its $\square$-graded homology groups

$$
\mathrm{GFH}^{(a, b)}(\overrightarrow{\mathcal{H}}, J)=\operatorname{Ker} d / \operatorname{Im} d
$$

are called generalized Floer homology of the framed Hamiltonian structure $\overrightarrow{\mathcal{H}}$. As we will see below (see Proposition 4.30) the homology is independent of $J$ and of the choice of a framing.

Suppose that a diffeomorphism $\Phi: V \rightarrow V$ is an equivalence between two framed Hamiltonian structures $\overrightarrow{\mathcal{H}}_{1}$ and $\overrightarrow{\mathcal{H}}_{2}$. Let $J_{1}$ be an almost complex structure adjusted to $\overrightarrow{\mathcal{H}}_{1}$, and $J_{2}=\Phi_{*}\left(J_{1}\right)$. Then $\Phi$ induces a canonical isomorphism

$$
\Phi_{\sharp}: \mathrm{GFH}^{(a, b)}\left(\overrightarrow{\mathcal{H}}_{1}, J_{1}\right) \rightarrow \mathrm{GFH}^{(a, b)}\left(\overrightarrow{\mathcal{H}}_{2}, J_{2}\right) .
$$

Indeed, $\Phi$ sends periodic orbits to periodic orbits, preserves their action and establishes a diffeomorphism between the moduli spaces of $J_{1}$ - and $J_{2}$-holomorphic curves. Furthermore, $\Phi$ induces a natural bijection between the grading sets $\square\left(\mathcal{H}_{1}\right)$ and $\square\left(\mathcal{H}_{2}\right)$. Isomorphism $\Phi_{\sharp}$ alters the grading of generalized Floer homology in agreement with this bijection.

The generalized Floer homology are equipped with canonical varying window homomorpisms

$$
E\left(a, a^{\prime}\right): \mathrm{GFH}^{\left(a^{\prime}, b\right)} \rightarrow \mathrm{GFH}^{(a, b)} \text { and } E\left(b, b^{\prime}\right): \mathrm{GFH}^{(a, b)} \rightarrow \mathrm{GFH}^{\left(a, b^{\prime}\right)}
$$

for $a^{\prime}<a$ and $b<b^{\prime}$. These homomorphisms preserve the grading.

\subsection{Directed concordance of Hamiltonian structures}

4.8.1 Concordance Let $\mathcal{H}_{+}=\left(\Theta_{+}\right)$and $\mathcal{H}_{-}=\left(\Theta_{-}\right)$be two Hamiltonian structures on $V$. We write $\mathrm{C}_{ \pm}, \mathrm{K}_{ \pm}$for the corresponding structure constants. The standing assumption of this subsection is $\mathrm{C}_{+}>\mathrm{C}_{-}$. 
Definition 4.19 A directed concordance between a pair of Hamiltonian structures $\mathcal{H}_{-}$ and $\mathcal{H}_{+}$is a pair $(W,(\Pi))$, where $(\Pi)$ is an equivalence class of 1 -forms on the manifold $W=V \times\left[a_{-}, a_{+}\right]$and $a_{-}<a_{+}$are any real numbers, such that

(i) $\left.\Pi\right|_{\partial_{ \pm} W}=\Theta_{ \pm}$, where $\partial_{ \pm} W=V \times a_{ \pm} ;$

(ii) $d \Pi$ is a symplectic form on $W$;

(iii) $\Pi=f(s) d t-g(s) \alpha$ outside $(V \backslash Z) \times\left[a_{-} ; a_{+}\right]$, where $f$ and $g$ are real valued functions with $f^{\prime}>0$ and $g>0$, and $Z \subset V$ is a compact set.

The conditions $f^{\prime}>0, g>0$ guarantee that the 2 -form $d \Pi$ is symplectic outside $Z \times\left[a_{-}, a_{+}\right]$. The assumption $\mathrm{C}_{+}>\mathrm{C}_{-}$is a consequence of $f^{\prime}>0$. We call any 1-form $\Pi$ representing $(\Pi)$ and satisfying condition (iii) the concordance structure.

We will sometimes denote a directed concordance as $\operatorname{Con}\left(\mathcal{H}_{-}, \mathcal{H}_{+}\right)$. Let us emphasize that in this notation $\mathcal{H}_{-}$is the Hamiltonian structure on the bottom of the concordance and $\mathcal{H}_{+}$is on its top, where the vertical direction is oriented by the variable $s$.

Example 4.20 We work in the situation described in Example 4.4 above. Let $\lambda_{ \pm}=$ $F_{ \pm}(d t-\alpha)$ be two admissible contact forms on $V$ with $F_{+}>F_{-}$. Let $S V=V \times \mathbb{R}_{+}$ be the symplectization of $V$ endowed with the Liouville form $\Pi=s(d t-\alpha)$. Consider the domain

$$
U=\left\{F_{-}(x, t) \leq s \leq F_{+}(x, t)\right\}
$$

and define a diffeomorphism $\varphi: V \times[0,1] \rightarrow U$ by the formula

$$
\varphi(x, t, s)=\left(x, t, F_{-}(x, t)+s\left(F_{+}(x, t)-F_{-}(x, t)\right)\right) .
$$

One readily checks that $\left(V \times[0,1],\left(\varphi^{*} \Pi\right)\right)$ is a concordance between the Hamiltonian structures $\mathcal{H}_{\lambda_{+}}=\left(V,\left(\lambda_{+}\right)\right)$and $\mathcal{H}_{\lambda_{-}}=\left(V,\left(\lambda_{-}\right)\right)$.

Example 4.21 We work in the situation described in Example 4.5 above. Let $F_{+}$and $F_{-}$be two time-dependent Hamiltonian functions on $M$ with $F_{+}>F_{-}$. Consider the domain

$$
U=\left\{F_{-}(x, t) \leq s \leq F_{+}(x, t)\right\} \subset V \times \mathbb{R}
$$

endowed with the 1 -form $\Pi=s d t-\alpha$. Let $\varphi$ be the diffeomorphism $V \times[0,1] \rightarrow U$ defined by formula (52). Then $\left(V \times[0,1],\left(\varphi^{*} \Pi\right)\right)$ is a concordance between the Hamiltonian structures $\mathcal{H}_{F_{+}}$and $\mathcal{H}_{F_{-}}$.

It will be convenient in the sequel to deal with equivalence classes of concordances. To define it let us stick to the splitting $V=M \times S^{1}$. 
Definition 4.22 Two concordances

$$
\left(W_{1}=V \times\left[a_{-} ; a_{+}\right],\left(\Pi_{1}\right)\right) \quad \text { and } \quad\left(W_{2}=V \times\left[b_{-}, b_{+}\right],\left(\Pi_{2}\right)\right)
$$

are called equivalent if there is a diffeomorphism $\phi: W_{1} \rightarrow W_{2}$ such that

(i) $\left(\phi^{*} \Pi_{2}\right)=\left(\Pi_{1}\right)$;

(ii) $\phi\left(z, t, a_{ \pm}\right)=\left(z, t, b_{ \pm}\right)$for all $(z, t) \in M \times S^{1}$;

(iii) there exists a diffeomorphism $u:\left[a_{-}, a_{+}\right] \rightarrow\left[b_{-}, b_{+}\right]$with $u\left(a_{ \pm}\right)=b_{ \pm}$and a positive function $v:\left[a_{-}, a_{+}\right] \rightarrow \mathbb{R}_{+}$with $v\left(a_{-}\right)=v\left(a_{+}\right)=1$ such that $\phi(z, t, s)=(v(s) * z, t, u(s))$ for every point $(z, t)$ lying outside a compact subset of $V$. Here $c * z$ stands for the natural $\mathbb{R}_{+}$-action on $V$ generated by the Liouville field.

As an immediate consequence of the Darboux theorem, we have the following useful lemma:

Lemma 4.23 (Normal form) Let $\overrightarrow{\mathcal{H}}_{ \pm}=\left(\left(\Theta_{ \pm}\right), \lambda_{ \pm}\right)$be framed Hamiltonian structures and let Con be any concordance between $\mathcal{H}_{+}$and $\mathcal{H}_{-}$. Then Con is equivalent to a concordance $\left(W=V \times\left[a_{+}, a_{-}\right],(\Pi)\right)$ such that the concordance structure $\Pi$ satisfies the conditions

- $\Pi=\Theta_{+}+\left(s-a_{+}\right) \lambda_{+}$on $V \times\left(a_{+}-\varepsilon, a_{+}\right]$

- $\Pi=\Theta_{-}+\left(s-a_{-}\right) \lambda_{-}$on $V \times\left[a_{-}, a_{-}+\varepsilon\right)$

for some $\varepsilon>0$.

Let us emphasize that this normal form depends on a choice of the framings $\lambda_{ \pm}$of $\mathcal{H}_{ \pm}$.

4.8.2 Concordances and coherent trivializations Let $e \in \mathcal{S}$ be a free homotopy class of loops $S^{1} \rightarrow V$. Every directed concordance $\operatorname{Con}\left(\mathcal{H}_{-}, \mathcal{H}_{+}\right)$induces a canonical bijection $\kappa_{e}: \mathbb{J}_{e}\left(\mathcal{H}_{+}\right) \rightarrow \mathbb{J}_{e}\left(\mathcal{H}_{-}\right)$between the sets of coherent trivializations in the class $e$ associated to Hamiltonian structures on its top and on its bottom. Moreover, $\kappa_{e}$ is equivariant with respect to the canonical $\mathbb{Z}$-actions on $\rrbracket_{e}\left(\mathcal{H}_{+}\right)$and $\rrbracket_{e}\left(\mathcal{H}_{-}\right)$. In fact, this bijection depends only on the equivalence classes of concordances. The bijection $\kappa_{e}$ is defined as follows. Let $\left(W=V \times\left[a_{-}, a_{+}\right],(\Pi)\right)$ be our concordance. Denote by $\ell_{ \pm}$the characteristic line fields of $\mathcal{H}_{ \pm}$. Choose framings of $\mathcal{H}_{-}$and $\mathcal{H}_{+}$and denote by $R_{ \pm} \in \ell_{ \pm}$the corresponding Reeb vector fields. Set $F_{ \pm}=\operatorname{Span}\left(\frac{\partial}{\partial s}, R_{ \pm}\right)$, and let $E_{ \pm}$be the skew-orthogonal complements (in the sense of the symplectic form $d(\Pi))$ of $F_{ \pm}$at the points of $\partial_{ \pm} W$. Clearly, $E_{ \pm}$is naturally identified with $T V / \ell_{ \pm}$. 
Let $\phi_{ \pm}: S^{1} \rightarrow V \times\left\{a_{ \pm}\right\}$be two loops representing $e$. Every trivialization $g_{ \pm}$of $\phi_{ \pm}^{*} E_{ \pm}$gives rise to a uniquely defined trivialization $h_{ \pm}$of $\phi_{ \pm}^{*}(T W)$. Indeed, at the points of $\partial_{ \pm} W$ we have $T W=E_{ \pm} \oplus F_{ \pm}$, where the bundle $F_{ \pm}$is equipped with a basis and hence is a priori trivialized. In the case when $h_{+}$and $h_{-}$simultaneously extend to a trivialization of $T W$ over a cylinder in $W$ spanning $\phi_{+}$and $\phi_{-}$, we say that $\kappa_{e}\left(\left[g_{+}\right]\right)=\left[g_{-}\right]$. One readily checks that the map $\kappa$ is a well defined equivariant bijection. It gives rise to an equivariant bijection $\chi_{e}: \rrbracket_{e}\left(\mathcal{H}_{+}\right) \rightarrow \rrbracket_{e}\left(\mathcal{H}_{-}\right)$between the grading sets in the class $e$. Setting $\chi(e, i)=\left(e, \chi_{e}(i)\right)$ we get a bijection $\chi: \llbracket\left(\mathcal{H}_{+}\right) \rightarrow$ $\llbracket\left(\mathcal{H}_{-}\right)$between the full grading sets.

Assume now that $\gamma_{+}$and $\gamma_{-}$are closed orbits of $R_{+}$and $R_{-}$in the class $e$. Then the difference $\mathrm{CZ}\left(\gamma_{+}, j\right)-\mathrm{CZ}\left(\gamma_{-}, \kappa_{e}(j)\right)$ does not depend on the particular choice of a coherent trivialization $j \in \mathbb{J}_{e}\left(\mathcal{H}_{+}\right)$. Thus we will denote this difference by $\mathrm{CZ}\left(\gamma_{+}\right)-\mathrm{CZ}\left(\gamma_{-}\right)$.

In the situation described in Remark 4.10, that is when $e$ is the class of point $\times S^{1}$, the map $\kappa$ preserves the distinguished element. As far as the case $M=T^{*} X$ is concerned (see Remark 4.11) the same holds true for all $e \in \mathcal{S}$ for the concordances which will arise in the present paper. We will use this without special mentioning.

Remark 4.24 Note that every homotopy $\Theta_{t}, t \in[0,1]$, between $\mathcal{H}_{0}=\left(\Theta_{0}\right)$ and $\mathcal{H}_{1}=\left(\Theta_{1}\right)$ through Hamiltonian structures gives rise to a natural equivariant bijection between the grading sets of this structures. In order to include this situation in the context of concordances we say that a concordance $\left(V \times\left[a_{-}, a_{+}\right],(\Pi)\right)$ is homotopylike if the restriction of $\Pi$ to each hypersurface $V \times\{s\}$, where $s \in\left[a_{-}, a_{+}\right]$, is a Hamiltonian structure. One can easily check that for a homotopy-like concordance the bijection associated to the homotopy $\left(\left.\Pi\right|_{V \times\{s\}}\right)$ of Hamiltonian structures coincides with the bijection $\chi$ introduced in this section. For instance, when $\Theta_{1}$ is sufficiently close to $\Theta_{0}$, the linear segment between these 1-forms passes through Hamiltonian structures (see Proposition 4.3 and its proof). Therefore, in this case the full grading sets of $\mathcal{H}_{0}$ and $\mathcal{H}_{1}$ are naturally identified.

4.8.3 Adjusted almost complex structures on concordances Let $\overrightarrow{\mathcal{H}}_{ \pm}$be two framed Hamiltonian structures. Choose adjusted almost-complex structures $J_{ \pm}$for $\overrightarrow{\mathcal{H}}_{ \pm}$associated to the splittings

$$
M \backslash \operatorname{Core}(M)=P_{ \pm} \times \mathbb{R},
$$

where $P_{ \pm} \subset M$ are starshaped hypersurfaces. Let

$$
\text { Con }=\left(W=M \times S^{1} \times\left[a_{-}, a_{+}\right],(\Pi)\right)
$$


be a directed concordance between $\mathcal{H}_{+}$and $\mathcal{H}_{-}$. We assume that $W$ lies in $\bar{V}=V \times \mathbb{R}$. Fix real numbers

$$
a_{+}^{\prime \prime}>a_{+}^{\prime}>a_{+} \text {and } a_{-}^{\prime \prime}<a_{-}^{\prime}<a_{-} .
$$

Choose a smooth family $P_{s}, s \in\left(a_{-}^{\prime \prime}, a_{+}^{\prime \prime}\right)$, of starshaped hypersurfaces in $M$ such that $P_{s}=P_{+}$for $s>a_{+}^{\prime}$ and $P_{s}=P_{-}$for $s<a_{-}$. Denote by $c * z$, where $c \in \mathbb{R}_{+}$and $z \in M$, the natural $\mathbb{R}_{+}$-action defined by the Liouville field $L$ on $M$. Hypersurfaces

$$
\Sigma_{c}=\bigcup_{s \in\left(a_{-}^{\prime \prime}, a_{+}^{\prime \prime}\right)}\left(c * P_{s}\right) \times S^{1} \times\{s\},
$$

$c \in \mathbb{R}_{+}$, foliate the set

$$
(M \backslash \operatorname{Core}(M)) \times S^{1} \times\left(a_{-}^{\prime \prime}, a_{+}^{\prime \prime}\right) .
$$

Denote by $S$ the vector field on this set obtained as the projection of $\frac{\partial}{\partial s}$ to $T \Sigma_{c}$ along the Liouville field $L$. Let $f(s), g(s)$ be the functions on $\left[a_{-}, a_{+}\right]$such that $f^{\prime}>0$, $g>0$ and $\Pi=f(s) d t-g(s) \alpha$ outside a compact subset of $W$ (see Definition 4.19(iii) above). Extend them to the interval $\left(a_{-}^{\prime \prime}, a_{+}^{\prime \prime}\right)$ keeping $f^{\prime}>0$ and $g>0$.

Denote by $\eta_{s}$ the natural contact structure on $P_{S}$, considered as a subbundle of $T W$.

Definition 4.25 An almost complex structure $I$ on $\bar{V}$ is called adjusted to the concordance Con if it satisfies

(i) $I=J_{+}$on $\left\{s>a_{+}^{\prime}\right\}$ and $I=J_{-}$on $\left\{s<a_{-}^{\prime}\right\}$;

(ii) there exists a 1 -form $\tilde{\Pi}$ on $V \times\left(a_{-}^{\prime \prime}, a_{+}^{\prime \prime}\right)$ so that $(\widetilde{\Pi})=(\Pi)$ on $W$ and $d \widetilde{\Pi}$ is symplectic form which tames $I$.

In addition, we request that there exists a compact subset $Z \subset V$ such that on ( $V \backslash$ $Z) \times\left(a_{-}^{\prime \prime}, a_{+}^{\prime \prime}\right)$ the following conditions hold:

(iii) $\tilde{\Pi}=f(s) d t-g(s) \alpha$;

(iv) the plane field $\eta_{s}$ is invariant under $I$;

(v) $I S=k(s) \frac{\partial}{\partial t}$ for some function $k(s)>0$.

Conditions (i) and (v) are compatible provided $k(s)=1 / \mathrm{P}_{+}$on $\left(a_{+}^{\prime}, a_{+}^{\prime \prime}\right)$ and $k(s)=$ $1 / \mathrm{P}_{-}$on $\left(a_{-}^{\prime \prime}, a_{-}^{\prime}\right)$, where $\mathrm{P}_{ \pm}$are the structure constants of the framings of $\overrightarrow{\mathcal{H}}_{ \pm}$.

The space of almost complex structures adjusted to a concordance is contractible.

Note that the definition of an adjusted almost complex structure agrees with the notion of equivalence of concordances introduced above. Let $\operatorname{Con}^{\prime}=\left(W^{\prime},\left(\Pi^{\prime}\right)\right)$ be a concordance which is equivalent to Con $=(W,(\Pi))$ via some diffeomorphism $\phi: W \rightarrow W^{\prime}$. 
Assume that $I$ is an almost complex structure on $\bar{V}$ adjusted to concordance Con. Consider a piecewise smooth homeomorphism $\tilde{\psi}: \bar{V} \rightarrow \bar{V}$ which coincides with $\phi$ on $W$ and has the form $(z, t, s) \rightarrow\left(z, t, s+e_{ \pm}\right)$on the connected components of $\bar{V} \backslash W$ for suitable (unique!) constants $e_{ \pm}$. Let $U$ be a sufficiently small neighborhood of $W$ in $\bar{V}$. Smoothen $\tilde{\psi}$ inside $U \backslash W$ to a diffeomorphism $\psi: \bar{V} \rightarrow \bar{V}$. The smoothing can be performed in such a way that

$$
\psi(z, t, s)=(v(s) * z, t, u(s)) \text { on }(V \backslash K) \times\left(a_{-}^{\prime \prime}, a_{+}^{\prime \prime}\right),
$$

where the functions $u$ and $v$ are defined on $\left(a_{-}^{\prime \prime}, a_{+}^{\prime \prime}\right)$ and $K \subset V$ is a compact subset (see condition (iii) of Definition 4.22).

Lemma 4.26 The push-forward $I^{\prime}$ of $I$ under $\psi$ is adjusted to concordance Con' .

Proof The structure $I^{\prime}$ obviously satisfies conditions (i) and (ii) of Definition 4.25, where one defines $\widetilde{\Pi}^{\prime}$ as the push-forward of $\widetilde{\Pi}$ under $\psi$. Let us verify conditions (iii)-(v) "at infinity" using representation (53). Obviously $\widetilde{\Pi}^{\prime}$ satisfies (iii). Denote by $\psi_{\infty}$ the restriction of $\psi$ to $\bar{V}_{\infty}:=(V \backslash K) \times\left(a_{-}^{\prime \prime}, a_{+}^{\prime \prime}\right)$. Put $b_{ \pm}^{\prime \prime}=u\left(a_{ \pm}^{\prime \prime}\right), P_{s}^{\prime}=$ $v\left(u^{-1}(s)\right) * P_{u^{-1}(s)}$ and

$$
\Sigma_{c}^{\prime}=\bigcup_{s \in\left(b_{-}^{\prime \prime}, b_{+}^{\prime \prime}\right)}\left(c * P_{s}\right) \times S^{1} \times\{s\}
$$

where $c \in \mathbb{R}_{+}$. Then $\psi_{\infty}\left(\Sigma_{c}\right)=\Sigma_{c}^{\prime}$ for $c$ large enough. The distribution $\eta_{s}^{\prime}$, defined as the natural contact structure on $P_{s}^{\prime}$, is the image of $\eta_{s}$ under $\psi_{\infty}$ which guarantees (iv).

Finally, writing $L$ for the Liouville vector field on $M$, note that

$$
\left(\psi_{\infty}\right) * \frac{\partial}{\partial s}=\gamma(s) \frac{\partial}{\partial s}+\delta(s) L
$$

with $\gamma(s)>0$. Denote by $Y$ (respectively, $Y^{\prime}$ ) the projection of $T \bar{V}$ to $T \Sigma_{c}$ (respectively, to $T \Sigma_{c}^{\prime}$ ) along $L$ at points of $\bar{V}_{\infty}$ (respectively, of $\psi_{\infty}\left(\bar{V}_{\infty}\right)$ ). Observe that $\left(\psi_{\infty}\right)_{*}$ intertwines between $Y$ and $Y^{\prime}$. Put $S^{\prime}=Y^{\prime} \frac{\partial}{\partial s}$. Then, applying (54) we have

$$
I^{\prime} S^{\prime}=\frac{1}{\gamma(s)} I^{\prime} Y^{\prime}\left(\psi_{\infty}\right)_{*} \frac{\partial}{\partial s}=\frac{1}{\gamma(s)} I^{\prime}\left(\psi_{\infty}\right)_{*} Y \frac{\partial}{\partial s}=\frac{1}{\gamma(s)}\left(\psi_{\infty}\right)_{*} I S
$$

Using condition (v) for $I$ we conclude that

$$
I^{\prime} S^{\prime}=\frac{k(s)}{\gamma(s)}\left(\psi_{\infty}\right) * \frac{\partial}{\partial t}=\frac{k(s)}{\gamma(s)} \frac{\partial}{\partial t}
$$

which proves that $I^{\prime}$ satisfies condition (v). This completes the proof. 
The next result is an analogue of Lemma 4.14 in the context of concordances.

Lemma 4.27 Let an almost complex structure $I$ be adjusted to the directed concordance $(W,(\Pi))$. Then for a sufficiently large $c>0$ the hypersurface $\Sigma_{c}$ is weakly $I$-convex.

Proof Put $\zeta=\operatorname{Span}\left(S, \frac{\partial}{\partial t}\right)$. Assume that $c$ is large enough. Then

$$
T_{\mathbb{C}} \Sigma_{c}=\eta_{s} \oplus \zeta=\operatorname{Ker}\left(\left.\alpha\right|_{T \Sigma_{c}}\right) .
$$

Clearly, $d \alpha=0$ on $\zeta$. Thus it suffices to show that the 2-form $-d \alpha$ tames $I$ on $\eta_{s}$. For that purpose, note that near $\Sigma_{c}$ one has $\Pi=f(s) d t-g(s) \alpha$, and hence

$$
d \Pi=f^{\prime}(s) d s \wedge d t-g^{\prime}(s) d s \wedge \alpha-g(s) d \alpha .
$$

Thus $\left.d \Pi\right|_{\eta_{s}}=-\left.g(s) d \alpha\right|_{\eta_{s}}$. By definition, $d \Pi$ tames $I$, and therefore, $-d \alpha$ tames $I$ on $\eta_{s}$. This yields the desired weak convexity.

Remark 4.28 Let $\overrightarrow{\mathcal{H}}_{ \pm}$be two framed Hamiltonian structures, and let Con $=(V \times$ $\left.\left[a_{-}, a_{+}\right],(\Pi)\right)$ be a concordance between them. Assume that the concordance is in the normal form as in Lemma 4.23. Let $J_{ \pm}$be almost complex structures on the top and on the bottom adjusted to $\overrightarrow{\mathcal{H}}_{ \pm}$. Observe that $d \Pi$ tames $J_{+}$for $s \in\left(a_{+}-\varepsilon, a_{+}\right]$ and tames $J_{-}$for $s \in\left(a_{-}, a_{-}+\varepsilon\right]$ if $\varepsilon>0$ is small enough. Thus one can choose an almost complex structure $I$ on $V \times \mathbb{R}$ which is adjusted to Con, coincides with $J_{+}$for $s>a_{+}-\varepsilon$ and coincides with $J_{-}$for $s<a_{-}+\varepsilon$. We say that $I$ respects the normal form.

4.8.4 Gluing of concordances Suppose that we have three Hamiltonian structures $\mathcal{H}_{1}, \mathcal{H}_{2}$ and $\mathcal{H}_{3}$. Let

$$
\operatorname{Con}^{i+1, i}=\operatorname{Con}\left(\mathcal{H}_{i}, \mathcal{H}_{i+1}\right)=\left(W^{i+1, i},\left(\Pi^{i+1, i}\right)\right), i=1,2
$$

be directed concordances between $\mathcal{H}_{i+1}$ and $\mathcal{H}_{i}$. Up to equivalence of concordances we can assume that $\Pi^{i+1, i}$ are in normal forms with respect to some framings $\lambda_{i}$ on $\mathcal{H}_{i}$ (see Lemma 4.23 above) and $W^{i+1, i}=V \times\left[a_{i}, a_{i+1}\right]$ for $i=1,2$, where $a_{1}<a_{2}<a_{3}$. Then the formulas

$$
\begin{aligned}
& W^{31}=W^{32} \cup W^{21}=V \times\left[a_{1}, a_{3}\right] ; \\
& \Pi^{31}= \begin{cases}\Pi^{32} & \text { on } W^{32} \\
\Pi^{21} & \text { on } W^{21}\end{cases}
\end{aligned}
$$


define a smooth directed concordance $\operatorname{Con}^{31}=\left(W^{31},\left(\Pi^{31}\right)\right)$ between $\mathcal{H}_{3}$ and $\mathcal{H}_{1}$. which is called a composition of $\mathrm{Con}^{21}$ and $\mathrm{Con}^{32}$. We will denote this operation by

$$
\operatorname{Con}^{31}=\operatorname{Con}^{32} \diamond \operatorname{Con}^{21} .
$$

Note that $\Pi^{31}$ is automatically in a normal form for the framed Hamiltonian structures $\overrightarrow{\mathcal{H}}_{3}$ and $\overrightarrow{\mathcal{H}}_{1}$. One readily checks that the equivalence class of a directed concordance $\mathrm{Con}^{31}$ is determined by the equivalence classes of $\mathrm{Con}^{32}$ and $\mathrm{Con}^{21}$.

Assume now that our framed Hamiltonian structures $\overrightarrow{\mathcal{H}}_{i}$ are equipped with adjusted almost complex structures $J_{i}$. Assume further that the concordances $\operatorname{Con}^{21}$ and $\operatorname{Con}^{32}$ are equipped with adjusted almost complex structures $I^{21}$ and $I^{32}$ which respect the almost complex structures on the boundaries and which respect the normal forms of the concordances (see Remark 4.28 above). Define an almost complex structure $I^{31}$ on $V \times \mathbb{R}$ which coincides with $I^{21}$ for $s \geq a_{2}$ and with $I^{32}$ for $s \leq a_{2}$. One readily checks that $I^{31}$ is smooth, adjusted to $\operatorname{Con}^{31}$ and respects $J_{1}, J_{3}$ and the normal form of $\mathrm{Con}^{31}$.

\subsection{Directed concordances as morphisms in Floer Homology}

4.9.1 Monotonicity homomorphism Let $\overrightarrow{\mathcal{H}}_{ \pm}$be a pair of framed Hamiltonian structures together with a directed concordance $\operatorname{Con}=(W,(\Pi))$ between them. Let $\chi: \llbracket\left(\mathcal{H}_{+}\right) \rightarrow \llbracket\left(c H_{-}\right)$be the bijection between the grading sets on the top and the bottom of the concordance introduced in Section 4.8.2. Choose adjusted almost complex structures $J_{ \pm}$and $I$ on $\bar{V}$. Denote by $\mathrm{C}_{ \pm}$the corresponding structure constants and by $\mathcal{P}_{ \pm}$the sets of periodic orbits of the Reeb vector fields $R_{ \pm}$(see Section 4.4).

Suppose we are given two orbits $\gamma_{ \pm} \in \mathcal{P}_{ \pm}$. Let us denote by $\mathcal{M}\left(\right.$ Con, $\left.I ; \gamma_{+}, \gamma_{-}\right)$the moduli space of proper $I$-holomorphic maps

$$
F=(h, \varphi): \Upsilon \rightarrow \bar{V}=V \times \mathbb{R}
$$

of the cylinder $\Upsilon=\mathbb{R} \times S^{1}$ which satisfy the following asymptotic conditions:

$$
\begin{aligned}
& \varphi(\sigma, \tau) \underset{\sigma \rightarrow \pm \infty}{\rightarrow}= \pm \infty, \\
& h(\sigma, \tau) \underset{\sigma \rightarrow \pm \infty}{\rightarrow}=\gamma_{ \pm}\left(T_{ \pm} \tau\right),
\end{aligned}
$$

where the orbits $\gamma_{ \pm}$are parameterized by the vector fields $R_{ \pm}$, and $T_{ \pm}$are their periods. Similarly to the cylindrical case we will impose the following generic regularity condition: the almost complex structure $I$ is regular along holomorphic cylinders. 
This implies, in particular, that for every pair of orbits $\gamma_{+}, \gamma_{-} \in \mathcal{P}_{ \pm}$with $\mathrm{CZ}\left(\gamma_{+}\right)-$ $\mathrm{CZ}\left(\gamma_{-}\right)=0$ we have

$$
\operatorname{dim} \mathcal{M}\left(\text { Con, } I, \gamma_{+}, \gamma_{-}\right)=0
$$

This enables us to define a monotonicity morphism

$$
\overline{\text { mon: }} C^{(a, b)}\left(\overrightarrow{\mathcal{H}}_{+}, J_{+}\right) \rightarrow C^{(a, b)}\left(\overrightarrow{\mathcal{H}}_{-}, J_{-}\right)
$$

by the formula

$$
\overline{\operatorname{mon}}\left(\gamma_{+}\right)=\sum_{\substack{\gamma \in \mathcal{P}^{(a, b)}\left(R_{+}\right) \\ \operatorname{cZ}\left(\gamma_{+}\right)-\operatorname{CZ}\left(\gamma_{-}\right)=0}} v\left(\gamma_{+}, \gamma_{-}\right) \gamma_{-},
$$

where $v\left(\gamma_{+}, \gamma_{-}\right)$is the mod 2 number of elements of the finite set $\mathcal{M}\left(\mathrm{Con}, I, \gamma_{+}, \gamma_{-}\right)$. This morphism respects the gradings of the complexes. The next standard fact from the Floer theory is as follows.

Theorem 4.29 Under the above assumptions, for any $a<b<\min \left(\mathrm{C}_{-}, \mathrm{C}_{+}\right)$and for all $(e, i) \in \llbracket\left(\mathcal{H}_{+}\right)$formula (57) defines a homomorphism of generalized Floer complexes

$$
\overline{\mathrm{mon}}:\left(C_{e, i}^{(a, b)}\left(\overrightarrow{\mathcal{H}}_{+}, J_{+}\right), d_{+}\right) \rightarrow\left(C_{\chi(e, i)}^{(a, b)}\left(\overrightarrow{\mathcal{H}}_{-}, J_{-}\right), d_{-}\right)
$$

so that we have

$$
d_{-} \circ \overline{\mathrm{mon}}=\overline{\mathrm{mon}} \circ d_{+} .
$$

In particular, $\overline{\text { mon }}$ defines a homology homomorphism

$$
\text { mon: } \mathrm{GFH}_{e, i}^{(a, b)}\left(\overrightarrow{\mathcal{H}}, J_{+}\right) \rightarrow \mathrm{GFH}_{\chi(e, i)}^{(a, b)}\left(\overrightarrow{\mathcal{H}}, J_{-}\right) \text {. }
$$

The homomorphism mon constructed above a priori depends on the following data:

- a concordance $\operatorname{Con}=\operatorname{Con}\left(\mathcal{H}_{-}, \mathcal{H}_{+}\right)=(W,(\Pi))$;

- an almost complex structure $I$ adjusted to Con which respects almost complex structures $J_{ \pm}$adjusted to $\overrightarrow{\mathcal{H}}_{ \pm}$.

Standard arguments of Floer theory show that the homomorphism mon does not change under the following operations:

- replacing the pair $(\mathrm{Con}, I)$ by a pair $\left(\mathrm{Con}^{\prime}, I^{\prime}\right)$ where $\mathrm{Con}^{\prime}$ is a concordance equivalent to Con and $I^{\prime}$ is the corresponding (see Lemma 4.26) almost complex structure adjusted to $\mathrm{Con}^{\prime}$;

- a homotopy of the concordance structure (П) on $W$ through the concordance structures; 
- a homotopy of the adjusted almost complex structure $I$ on $W$ through almost complex structures adjusted to Con and respecting $J_{ \pm}$.

Consider now three framed Hamiltonian structures $\overrightarrow{\mathcal{H}}_{1}, \overrightarrow{\mathcal{H}}_{2}$ and $\overrightarrow{\mathcal{H}}_{3}$ equipped with adjusted almost complex structures $J_{1}, J_{2}$ and $J_{3}$ respectively. Assume that we are given directed concordances

$$
\operatorname{Con}^{i+1, i}=\operatorname{Con}\left(\mathcal{H}_{i}, \mathcal{H}_{i+1}\right), i=1,2,
$$

which induce monotonicity morphisms

$$
\operatorname{mon}^{i+1, i}: \operatorname{GFH}\left(\overrightarrow{\mathcal{H}}_{i+1}, J_{i+1}\right) \rightarrow \mathrm{GFH}\left(\overrightarrow{\mathcal{H}}_{i}, J_{i}\right)
$$

A standard argument of Floer theory shows that the gluing $\operatorname{Con}^{32} \diamond \operatorname{Con}^{21}$ of concordances corresponds to the composition $\mathrm{mon}^{32} \circ \mathrm{mon}^{21}$ of the monotonicity morphisms.

The next result is crucial for our proof of non-squeezing results in contact geometry. It will enable us to reduce calculations in contact homology to more traditional calculations in Floer homology.

Proposition 4.30 For a regular Hamiltonian structure the generalized Floer homology does not depend on the choice of a framing and of an adjusted almost complex structure.

We start with two auxiliary lemmas whose proof is elementary and is left to the reader.

Lemma 4.31 Let $A_{i}^{\delta}$, where $\delta \in\{-, 0,+\}$ and $i \in\{0,1\}$ be a collection of six linear spaces. Suppose that we are given eight morphisms between them such that the following diagram commutes:

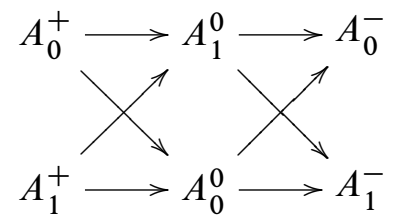

Assume that the diagonal arrows are isomorphisms. Then all the arrows are isomorphisms.

Lemma 4.32 Let $E$ be a topological linear space and let $\Lambda \subset E$ be a convex cone. Given any $\sigma_{0}, \sigma_{1} \in E$ with $\sigma_{1}-\sigma_{0} \in \operatorname{Interior}(\Lambda)$ and any $\lambda_{0}, \lambda_{1} \in \Lambda$, there exists a smooth path $\sigma_{s}, s \in[0,1]$, connecting $\sigma_{0}$ with $\sigma_{1}$ such that its derivative $\dot{\sigma_{s}}$ satisfies the following conditions:

(i) $\dot{\sigma}_{s}=\lambda_{0}$ for $s$ near 0 and $\dot{\sigma}_{s}=\lambda_{1}$ for $s$ near 1 ; 
(ii) $\dot{\sigma_{s}} \in \Lambda$ for all $s$.

Let us mention that it suffices to prove the lemma in dimension at most 3 by passing to $E^{\prime}=\operatorname{Span}\left(\sigma_{1}-\sigma_{0}, \lambda_{0}, \lambda_{1}\right)$ and $\Lambda^{\prime}=\Lambda \cap E^{\prime}$.

Let $(\Theta)$ be a Hamiltonian structure and let $\Lambda$ be the convex cone of its framings. Define the set $D$ of 1 -forms $\sigma=c \lambda$, where $c \in \mathbb{R}$ and $\lambda \in \Lambda$ so that $(\Theta+\sigma)$ is again a Hamiltonian structure. Recall that according to Axiom 3 for any $\lambda \in \Lambda$ there exists $\mu \geq 0$ such that $d \lambda=\mu d \Theta$. Hence, we have $-\epsilon \lambda \in D$ if $\mu \epsilon<1$. Then, given $\sigma \in D$ we have $d \sigma=\mu^{\prime} d \Theta$ with $1+\mu^{\prime}>0$, and hence Hamiltonian structures $(\Theta)$ and $(\Theta+\sigma)$ share the same characteristic foliation and the set of framings, and $\sigma+\Lambda \subset D$ for every $\sigma \in D$.

Suppose that we are given $\sigma_{0}, \sigma_{1} \in D$ such that $\sigma_{1}-\sigma_{1} \in \operatorname{Interior}(\Lambda)$. For any $\lambda_{0}, \lambda_{1} \in \Lambda$ consider framed Hamiltonian structures $\overrightarrow{\mathcal{H}}_{i}=\left(\left(\Theta+\sigma_{i}\right), \lambda_{i}\right)$, where $i \in\{0,1\}$, equipped with adjusted almost complex structures $J_{i}$. We wish to associate to these data a monotonicity morphism

$$
\text { mon: } \mathrm{GFH}\left(\overrightarrow{\mathcal{H}}_{1}, J_{1}\right) \rightarrow \mathrm{GFH}\left(\overrightarrow{\mathcal{H}}_{0}, J_{0}\right)
$$

associated to a certain directed concordance $(V \times[0,1],(\Pi))$ between $\mathcal{H}_{0}$ and $\mathcal{H}_{1}$ equipped with an adjusted almost complex structure $I$ which respects $J_{0}$ and $J_{1}$. Here the form $\Pi$ is defined by $\Pi=\Theta+\sigma_{s}$, where the family of 1 -forms $\sigma_{s}$ is chosen from Lemma 4.32. Since $\sigma_{s} \in D$ for all $s$ in view of condition (ii) of the lemma, we have $d \sigma_{s}=\mu_{s} d \Theta$ with $1+\mu_{s}>0$. Then the 2 -form

$$
d \Pi=\left(1+\mu_{s}\right) d \Theta+d s \wedge \dot{\sigma_{s}}
$$

is symplectic since the 1 -form $\dot{\sigma_{s}}$ lies in $\Lambda$ and hence is positive on the characteristic foliation of $d \Theta$. Take $\kappa>0$ small enough and define an almost complex structure $I$ on $V \times \mathbb{R}$ as $J_{0}$ for $s<\kappa$ and as $J_{1}$ for $s>1-\kappa$. It follows from condition (i) of Lemma 4.32 that $d \Pi$ tames $I$ near the top and the bottom of our concordance. Extending $I$ in an arbitrary way to an adjusted almost complex structure on $V \times \mathbb{R}$ which is tamed by $d \Pi$, we complete the construction. For the sake of further reference, we call the constructed morphism the preparatory morphism.

Remark 4.33 Assume that in the above setting $\lambda_{0}=\lambda_{1}$ and $J_{0}=J_{1}=J$. Then the adjusted almost complex structure $I$ on the constructed concordance can be chosen as $I=J$. In this case all $I$-holomorphic cylinders with 0 -dimensional moduli spaces are of the form $\gamma \times \mathbb{R}$ where $\gamma$ is a closed orbit of the common characteristic foliation of $\mathcal{H}_{0}$ and $\mathcal{H}_{1}$. Therefore, our concordance induces the identity morphism in the 
generalized Floer chain complexes $C F\left(\overrightarrow{\mathcal{H}}_{1}, J\right)$ and $C F\left(\overrightarrow{\mathcal{H}}_{0}, J\right)$. Let $a<b$ be noncritical values of the Hamiltonian structure $(\Theta)$ so that the intervals $(a-\kappa, a+\kappa)$ and $(b-\kappa ; b+\kappa)$ do not intersect the action spectrum $\operatorname{spec}((\Theta))$ for some $\kappa>0$ small enough. Suppose now that the 1 -forms $\sigma_{0}$ and $\sigma_{1}$ are sufficiently small. Then the sets $\mathcal{P}^{(a, b)}\left(\mathcal{H}_{1}\right)$ and $\mathcal{P}^{(a, b)}\left(\mathcal{H}_{0}\right)$ consist of the same periodic orbits. Thus our concordance gives an isomorphism between $\mathrm{GFH}^{(a, b)}\left(\overrightarrow{\mathcal{H}}_{1}, J\right)$ and $\mathrm{GFH}^{(a, b)}\left(\overrightarrow{\mathcal{H}}_{0}, J\right)$.

Proof of Proposition 4.30 Let $a<b$ be non-critical values of the Hamiltonian structure $(\Theta)$. Let $\lambda_{0}, \lambda_{1}$ be two different framings of the Hamiltonian structure $(\Theta)$, and $J_{i}$ an almost complex structure on $V \times \mathbb{R}$ adjusted to the framed Hamiltonian structure $\overrightarrow{\mathcal{H}}_{i}^{0}=\left((\Theta), \lambda_{i}\right), i=0,1$. Pick any framing $\lambda \in \operatorname{Interior}(\Lambda)$, and take $\epsilon>0$ small enough so that $-\varepsilon \lambda$ lies in the set $D$. Consider framed Hamiltonian structures

$$
\overrightarrow{\mathcal{H}}_{i}^{ \pm}=\left((\Theta \pm \varepsilon \lambda), \lambda_{i}\right), \quad i \in\{0,1\},
$$

and put

$$
A_{i}^{\delta}:=\mathrm{GFH}^{(a, b)}\left(\overrightarrow{\mathcal{H}}_{i}^{\delta}, J_{i}\right), \quad \delta \in\{-, 0,+\}, i \in\{0,1\} .
$$

Consider the diagram (58) whose arrows are defined as the preparatory morphisms. One can easily check that this diagram is commutative: in order to prove commutativity of a parallelogram formed by its arrows, one has to verify that the compositions of the concordances corresponding to these arrows have homotopic concordance data. We leave the details to the reader. Decreasing if necessary $\epsilon$, we get that the diagonal arrows are isomorphisms, see Remark 4.33. Hence by Lemma 4.31 all the arrows are isomorphisms and in particular the spaces $A_{0}^{0}=\mathrm{GFH}^{(a, b)}\left((\Theta), \lambda_{0}\right)$ and $A_{1}^{0}=$ $\mathrm{GFH}^{(a, b)}\left((\Theta), \lambda_{1}\right)$ are isomorphic. Note that these isomorphisms preserve the grading since all the concordances involved into their construction are homotopy-like in the sense of Remark 4.24 above. This completes the proof.

Remark 4.34 One can readily check that the isomorphism between generalized Floer homologies provided by Proposition 4.30 is canonical in the following sense. Let $\lambda_{i}$, $i \in\{1,2,3\}$, be three framings of $(\Theta)$ and let $J_{i}$ be almost complex structures adjusted to $\overrightarrow{\mathcal{H}}_{i}=\left((\Theta), \lambda_{i}\right)$. Let

$$
F_{i, j}: \mathrm{GFH}\left(\overrightarrow{\mathcal{H}}_{j}, J_{j}\right) \rightarrow \mathrm{GFH}\left(\overrightarrow{\mathcal{H}}_{i}, J_{i}\right), i, j \in\{1,2,3\},
$$

be our isomorphism. Then $F_{13}=F_{12} \circ F_{23}$.

It follows from Proposition 4.30 and Remark 4.34 that we can remove the framing and the almost complex structure from the notation and write $\mathrm{GFH}^{(a, b)}(\mathcal{H})$ for the generalized Floer homology of a Hamiltonian structure $\mathcal{H}$. 
Consider now two regular Hamiltonian structures $\mathcal{H}_{0}=\left(\Theta_{0}\right)$ and $\mathcal{H}_{1}=\left(\Theta_{1}\right)$. Recall that the full grading sets of sufficiently close Hamiltonian structures can be naturally identified (see Remark 4.24).

Proposition 4.35 Take any two non-critical values $a<b$ of $\mathcal{A}_{\mathcal{H}_{0}}$. If $\Theta_{1}$ is sufficiently close to $\Theta_{0}$, there is a grading-preserving isomorphism

$$
\mathrm{GFH}^{(a, b)}\left(\mathcal{H}_{0}\right) \rightarrow \mathrm{GFH}^{(a, b)}\left(\mathcal{H}_{1}\right)
$$

Proof Fix a framing $\lambda_{0}$ of $\mathcal{H}_{0}$ with the framing parameter $\mu$. Then $\lambda_{1}=\lambda_{0}+$ $\mu\left(\Theta_{1}-\Theta_{0}\right)$ is a framing of $\mathcal{H}_{1}$. Take $\epsilon>0$ small enough so that $\left(\Theta_{0}-\varepsilon \lambda_{0}\right)$ is a Hamiltonian structure. If $\Theta_{1}$ is sufficiently close to $\Theta_{0}$, the 1-form $\Theta_{1}-\varepsilon \lambda_{1}$ also defines a Hamiltonian structure. Consider Hamiltonian structures $\mathcal{H}_{i}^{ \pm}=\left(\Theta_{i} \pm \varepsilon \lambda_{i}\right)$, where $i \in\{0,1\}$. Re-denote $\mathcal{H}_{i}^{0}:=\mathcal{H}_{i}$ and put

$$
A_{i}^{\delta}:=\mathrm{GFH}^{(a, b)}\left(\mathcal{H}_{i}^{\delta}\right), \quad \delta \in\{-, 0,+\}, i \in\{0,1\} .
$$

Our next goal to describe the arrows in the diagram (58). The diagonal arrows are defined as the isomorphisms presented in Remark 4.33. The horizontal arrows are associated to the following directed concordances between the corresponding Hamiltonian structures. The concordance structure $\Pi$ on each of these concordances corresponds to the linear segment between the 1-forms defining the Hamiltonian structures. Let us work out the case of the upper left horizontal arrow $A_{0}^{+} \rightarrow A_{1}^{0}$ (all other cases are absolutely similar): Here the 1 -form $\Pi$ on $V \times[0,1]$ is defined as

$$
\Pi=\Theta_{1}+s\left(\Theta_{0}+\varepsilon \lambda_{0}-\Theta_{1}\right) .
$$

We claim that $d \Pi$ is symplectic. Indeed,

$$
d \Pi=(1+\varepsilon \mu s) d \Theta_{0}+\varepsilon d s \wedge \lambda_{0}+d s \wedge\left(\Theta_{0}-\Theta_{1}\right)-(1-s)\left(d \Theta_{0}-d \Theta_{1}\right)
$$

is a small perturbation of the non-degenerate 2 -form $(1+\varepsilon \mu s) d \Theta_{0}+\varepsilon d s \wedge \lambda_{0}$ provided that $\Theta_{1}$ is sufficiently close to $\Theta_{0}$, and hence it is non-degenerate as well. The claim follows.

One readily checks that we got a commutative diagram, and hence Lemma 4.31 yields the desired isomorphism. It preserves the grading since all the concordances involved in the construction of this isomorphism are homotopy-like in the sense of Remark 4.24 above.

We complete this section with mentioning that the monotonicity morphisms behave naturally with respect to diffeomorphisms. Assume that $\mathcal{H}_{i}^{ \pm}, i=0,1$, are four 
Hamiltonian structures and

$$
\operatorname{Con}\left(\mathcal{H}_{0}^{-}, \mathcal{H}_{0}^{+}\right)=\left(W_{0},\left(\Pi_{0}\right)\right) \text { and } \operatorname{Con}\left(\mathcal{H}_{1}^{-}, \mathcal{H}_{1}^{+}\right)=\left(W_{1},\left(\Pi_{1}\right)\right)
$$

are two directed concordances. Let $A: W_{0} \rightarrow W_{1}$ be a diffeomorphism whose restrictions to the boundaries $\partial_{ \pm} W_{0}$ send $\mathcal{H}_{0}^{ \pm}$to $\mathcal{H}_{1}^{ \pm}$and such that $\left(A^{*} \Pi_{1}\right)=\left(\Pi_{0}\right)$. Then the following diagram commutes:

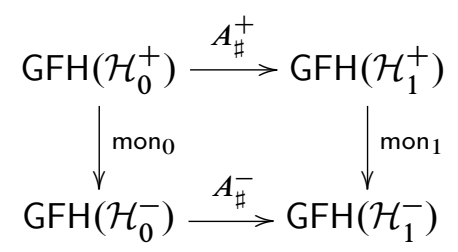

where the vertical arrows correspond to the monotonicity morphisms defined by the concordances, and the horizontal arrows stand for the natural morphisms induced by the restriction of $A$ to $\partial_{ \pm} W_{0}$.

\subsection{GFH in the absence of regularity}

Let $\mathcal{H}=(\Theta)$ be a Hamiltonian structure, not necessarily regular. For our applications, it would be convenient to extend the definition of generalized Floer homology $\mathrm{GFH}^{(a, b)}(\mathcal{H})$ to this case. This is done as follows (in fact, we imitate analogous construction in the context of usual Floer homology). Assume that $a<b$ are noncritical values of $\mathcal{A}_{\mathcal{H}}$. Take a sufficiently small regular perturbation $\Theta_{0}$ of $\Theta$ defining a Hamiltonian structure $\mathcal{H}_{0}=\left(\Theta_{0}\right)$. Put

$$
\mathrm{GFH}^{(a, b)}(\mathcal{H})=\mathrm{GFH}^{(a, b)}\left(\mathcal{H}_{0}\right)
$$

Proposition 4.35 shows that if $\Theta_{0}$ and $\Theta_{1}$ is a pair of sufficiently small such perturbations, the spaces $\mathrm{GFH}^{(a, b)}\left(\mathcal{H}_{0}\right)$ and $\mathrm{GFH}^{(a, b)}\left(\mathcal{H}_{1}\right)$ are canonically isomorphic and hence the definition above is correct. Similarly, one extends the monotonicity morphisms to not necessarily regular Hamiltonian structures.

\subsection{Discussion: non-special Hamiltonian structures}

Most of the above theory works for stable but not necessarily special Hamiltonian structures. Namely, suppose Axiom 3 in the definition of a special structure $\mathcal{H}=$ $(V,(\Theta))$ is weakened to simply the requirement of existence of a framing $\lambda \in F_{\mathrm{P}, \mathrm{Q}}$, ie, a 1 -form $\lambda$ such that $i_{R} d \lambda=R$ and $\lambda(R)=1$ for a characteristic vector field $R$ of $(\Theta)$ ) (see Section 4.1 above.) This leads to the following modifications: 
- The period-action equation (46) does not hold, and what is even worse, one does not necessarily have a bound on the period of a closed orbit in terms of its action.

- As a result we cannot prove analogues of Proposition 4.3 (openness of the stability condition) and of Proposition 4.7.

- Even if $\mathcal{H}$ is regular, it is not any longer true that the sets $\mathcal{P}^{a, b}$ for $a<b<\mathrm{C}$ are finite.

- However, assuming that $\mathcal{H}$ is regular, one can choose an adjusted almost complex structure $J$ and define the complex $\left(C^{(a, b)}(\overrightarrow{\mathcal{H}}, J), d\right)$ using the same formula (50) as in the special case. Though the complex $C^{(a, b)}(\overrightarrow{\mathcal{H}}, J)$ can be in this case infinitely generated, compactness results from [6] guarantee that the sum in formula (50) contains only finite number of terms, and hence, $\mathrm{GFH}(\overrightarrow{\mathcal{H}})$ is well defined in this case.

- Similarly, one can extend to this situation the definition of monotonicity homomorphisms and prove analogues of Theorems 4.29 and Proposition 4.30.

For certain applications (which we do not consider in this paper) it is important to further enlarge the class of admissible Hamiltonian structures. In particular, one can bypass Axiom 1, by considering Floer homology with coefficients in an appropriate Novikov ring. Without much changes one can relax Axiom 2 by requiring that there are no contractible periodic orbits of certain Maslov indices (cf [4; 44; 48]. Dropping Axiom 2 altogether leads to generalized Floer homology differential algebra rather than complex, in the spirit of [17].

\subsection{GFH for Hamiltonian functions}

4.12.1 (Generalized) Floer homology In this section we analyze Floer homology of Hamiltonian structures $\overrightarrow{\mathcal{H}}_{F}$ associated to a generic time-dependent Hamiltonian function $F(x, t)=F_{t}(x)$ on $M$ which equals to a positive constant $\mathrm{C}$ outside a compact set, see Example 4.5 above.

First of all, let us recall the standard definition of the Floer homology of $F$ (see eg [3]). The action functional $\mathcal{A}_{F}$ on the space of smooth loops $\gamma: S^{1} \rightarrow M$ is given by

$$
\mathcal{A}_{F}(\gamma)=\int_{0}^{1}-\gamma^{*} \alpha+F_{t}(\gamma(t)) d t .
$$

The values of $\mathcal{A}_{F}$ on 1-periodic orbits of the Hamiltonian flow generated by $F$ form the action spectrum $\operatorname{spec}(F)$. In what follows we refer to real numbers $a \in$ $(-\infty ; C) \backslash \operatorname{spec}(F)$ as to non-critical values of $\mathcal{A}_{F}$. 
Let $\bar{J}=\left\{J_{t}\right\}, t \in S^{1}$, be a family of almost complex structures compatible with $\omega=d \alpha$. Under certain generic assumptions, the filtrated Floer homology complex $C^{(a, b)}(F, \bar{J})$ is generated by 1 -periodic orbits $\bar{\gamma}_{i}$ of the Hamiltonian $F$ with

$$
a<\mathcal{A}_{F}\left(\bar{\gamma}_{i}\right)<b,
$$

where $a, b$ are non-critical values of $\mathcal{A}_{F}$.

Denote by $\mathcal{S}^{M}$ the set of free homotopy classes of loops $S^{1} \rightarrow M$. The generators $\bar{\gamma}_{i}$ of $C^{(a, b)}(F, \bar{J})$ are graded by their free homotopy classes $\varepsilon \in \mathcal{S}^{M}$ and by elements of the $\mathbb{Z}$-set $\square_{\varepsilon}^{M}:=\rrbracket_{\varepsilon}^{M} \times\{0,1\}$, where $\rrbracket_{\varepsilon}^{M}$ is the set of coherent symplectic trivializations of the bundle $(T M, \omega)$ over loops representing $\varepsilon$. The details of this construction are exactly the same as in Section 4.4.1 above, and we leave them to the reader. Put

$$
\rrbracket^{M}=\left\{(\varepsilon, i): \varepsilon \in \mathcal{S}^{M}, i \in \square_{\varepsilon}^{M}\right\} .
$$

Definition 4.36 The set $\rrbracket^{M}$ (respectively, $\square_{\varepsilon}^{M}$ ) is called the grading set of the Liouville manifold $(M, \omega)$ (respectively, in the class $\varepsilon$ ).

In the cases when $\varepsilon$ is the class of contractible loops or when $M$ is endowed with a Lagrangian distribution we get the canonical $\mathbb{Z}$-grading, as it was explained in Remarks 4.10 and 4.11 .

The boundary operator $d: C^{(a, b)}(F, \bar{J}) \rightarrow C^{(a, b)}(F, \bar{J})$ is defined by the formula

$$
d \bar{\gamma}_{i}=\sum_{i} v_{i j} \bar{\gamma}_{j}
$$

where $\mathrm{CZ}\left(\bar{\gamma}_{i}\right)-\mathrm{CZ}\left(\bar{\gamma}_{j}\right)=1$, and $v_{i j}$ is the number $(\bmod 2)$ of the trajectories of the reversed gradient flow $-\nabla \mathcal{A}_{F}$ on the loop space, connecting $\bar{\gamma}_{i}$ and $\bar{\gamma}_{j}$. More precisely, $v_{i j}$ counts components of the moduli space $\mathcal{M}_{i j}$ of maps $u: \mathbb{R} \times S^{1} \rightarrow M$ which satisfy an inhomogeneous Cauchy-Riemann equation

$$
\begin{aligned}
& \frac{\partial u}{\partial \sigma}(\sigma, \tau)+J_{\tau} \frac{\partial u}{\partial \tau}(\sigma, \tau)-J_{\tau} \operatorname{sgrad} F_{\tau}=0, \\
& \lim _{\sigma \rightarrow-\infty} u(\sigma, \tau)=\bar{\gamma}_{i}(\tau), \\
& \lim _{\sigma \rightarrow+\infty} u(\sigma, \tau)=\bar{\gamma}_{j}(\tau) .
\end{aligned}
$$

The homology $\mathrm{FH}(F)$ of the complex $\left(C^{(a, b)}(F, \bar{J}), d\right)$ is independent of $\bar{J}$ and called the Floer homology of the Hamiltonian $F$.

Let us turn now to the generalized Floer homology of the framed Hamiltonian structure $\overrightarrow{\mathcal{H}}_{F}$. Recall that the underlying Hamiltonian structure is given by the equivalence class 
of 1-forms $(\Theta)$ with $\Theta=F d t-\alpha$ on $V$, and the framing is chosen as $\lambda:=d t$. The 2-form $\Omega$ equals $d F_{t} \wedge d t-\omega$. Hence the Reeb vector field equals $R=\frac{\partial}{\partial t}+\operatorname{sgrad} F_{t}$, so that all its closed orbits are automatically non-contractible. Moreover, every orbit representing a class from $\mathcal{S}$ has period 1 .

The cut $\xi$ is the tangent bundle to the fibers $M \times$ \{point . Extend the family of almost complex structures $-J_{t}: T M \rightarrow T M$ compatible with $-\omega=\left.\Omega\right|_{\xi}$ (mind the minus sign!) to an adjusted almost complex structure $J$ on $\bar{V}=M \times S^{1} \times \mathbb{R}$. This means, in particular, that $J$ is invariant along the shifts $s \rightarrow s+c$, where $s$ is the coordinate on $\mathbb{R}$, and $J \frac{\partial}{\partial s}=R$. We assume that $J$ is regular.

Proposition 4.37 The graded filtrated Floer complexes $C^{(a, b)}\left(\overrightarrow{\mathcal{H}}_{F}, J\right)$ and $C^{(a, b)}(F, \bar{J})$ coincide together with their differentials. In particular, the (generalized) Floer homology $\mathrm{GFH}\left(\overrightarrow{\mathcal{H}}_{F}\right)$ and $\mathrm{FH}(F)$ are canonically isomorphic.

Proof The complex $C^{(a, b)}\left(\overrightarrow{\mathcal{H}}_{F}, J\right)$ is generated by periodic orbits $\gamma_{i}(t)$ of $R$ whose free homotopy classes lie in the set $\mathcal{S}$. Each such class, say $e$, has the form $e=$ $\varepsilon \oplus$ [point $\times S^{1}$ ] where $\varepsilon$ is a free homotopy class of loops $S^{1} \rightarrow M$. Write $\ell$ for the kernel of $\Omega$ and observe that the bundle $(T V / \ell, \Omega)$ can be canonically identified with $(T M,-\omega)$. Therefore, we have a canonical $\mathbb{Z}$-equivariant bijection between the sets $\rrbracket_{e}$ and $\rrbracket_{\varepsilon}^{M}$ of coherent trivializations, which yields an equivariant bijection between the grading sets $\rrbracket_{e}$ and $\square_{\varepsilon}^{M}$. The orbits $\gamma_{i}$ are in 1-1 correspondence with orbits $\bar{\gamma}_{i}$. Namely, each such orbit $\gamma_{i}: S^{1} \rightarrow M \times S^{1}$ has the form $\gamma_{i}(t)=\left(\bar{\gamma}_{i}(t), t\right)$. Moreover, we have $\operatorname{deg}\left(\gamma_{i}\right)=\operatorname{deg}\left(\overline{\gamma_{i}}\right)$ since the change of the sign of the symplectic form does not affect Conley-Zehnder indices. Furthermore,

$$
\mathcal{A}_{\mathcal{H}_{F}}\left(\gamma_{i}\right)=\int_{\gamma_{i}} F_{t} d t-\alpha=\int_{0}^{1}-\gamma_{i}^{*} \alpha+F_{t}\left(\gamma_{i}(t)\right) d t=\mathcal{A}_{F}\left(\bar{\gamma}_{i}\right) .
$$

Hence, the two complexes have the same grading and filtration. Now let us compare the differentials of these complexes. Consider the complex

$$
\left(C^{(a, b)}\left(\overrightarrow{\mathcal{H}}_{F}, J\right), d\right) \text {. }
$$

Recall from Section 4.7 that the coefficient at $\gamma_{j}$ in the expression for $d \gamma_{i}$ is determined by the 0 -dimensional component of the moduli space of $J$-holomorphic cylinders which are defined as follows. Write $\Upsilon=\mathbb{R} \times S^{1}$ for the standard cylinder endowed with coordinates $\sigma \in \mathbb{R}$ and $\tau \in S^{1}$. We endow $\Upsilon$ with the standard complex structure by introducing the complex coordinate $\sigma+i \tau$. The $\bar{\partial}$-equation

$$
\frac{\partial \Phi}{\partial \sigma}+J \frac{\partial \Phi}{\partial \tau}=0
$$


for a $J$-holomorphic cylinder $\Phi=(u, A, B): \Upsilon \rightarrow M \times S^{1} \times \mathbb{R}$ takes the form

$$
\begin{aligned}
& \frac{\partial A}{\partial \sigma}=-\frac{\partial B}{\partial \tau}, \\
& \frac{\partial A}{\partial \tau}=\frac{\partial B}{\partial \sigma} \\
& \frac{\partial u}{\partial \sigma}-J_{A(\sigma, \tau)} \frac{\partial u}{\partial \tau}+\frac{\partial A}{\partial \tau} J_{A(\sigma, \tau)} \operatorname{sgrad} F_{A(\sigma, \tau)}+\frac{\partial B}{\partial \tau} \operatorname{sgrad} F_{A(\sigma, \tau)}, \\
& \lim _{\sigma \rightarrow-\infty} u(\sigma, \tau)=\bar{\gamma}_{j}(\tau), \\
& \lim _{\sigma \rightarrow+\infty} u(\sigma, \tau)=\bar{\gamma}_{i}(\tau), \\
& \lim _{\sigma \rightarrow \pm \infty} A(\sigma, \tau)=\tau, \\
& \lim _{\sigma \rightarrow \pm \infty} B(\sigma, \tau)= \pm \infty .
\end{aligned}
$$

The first two equations just say that $B(\sigma, \tau)+i A(\sigma, \tau)$ is a holomorphic self-map of the cylinder, which together with the asymptotic boundary conditions imply that $A(\sigma, \tau)=\tau$ and $B(\sigma, \tau)=\sigma+c$ for some constant $c$. Without loss of generality we can assume that $c=0$. Thus the third equation can be rewritten as

$$
-\frac{\partial u}{\partial \sigma}(\sigma, \tau)+J_{\tau} \frac{\partial u}{\partial \tau}(\sigma, \tau)-J_{\tau} \operatorname{sgrad} F_{\tau}=0 .
$$

The change of variables $(\sigma, \tau) \mapsto(-\sigma, \tau)$ transforms the last equation together with the asymptotic boundary conditions to equation (60) arising in the usual Floer theory. Hence, we conclude that the Floer complexes $C^{(a, b)}\left(\overrightarrow{\mathcal{H}}_{F}, J\right)$ and $C^{(a, b)}(F, \bar{J})$ coincide together with their differentials.

Suppose now that we are given two Hamiltonian functions $G, F: M \times S^{1} \rightarrow \mathbb{R}$, $S^{1}=\mathbb{R} / \mathbb{Z}$, which are constant at infinity, and such that $G>F$. Then for any numbers $a<b$ which are non-critical values for both $\mathcal{A}_{F}$ and $\mathcal{A}_{G}$, in the Floer homology theory (see $[21 ; 37]$ ) there is defined a grading preserving monotonicity homomorphism mon $_{\mathrm{FH}}: \mathrm{FH}_{*}^{(a, b)}(G) \rightarrow \mathrm{FH}_{*}^{(a, b)}(F)$. On the other hand, the Hamiltonian structures $\mathcal{H}_{G}$ and $\mathcal{H}_{F}$ are concordant in a natural way, see Example 4.21 above. This concordance defines a homomorphism mon GFH $_{\text {GFH }}^{(a, b)}\left(\mathcal{H}_{G}\right) \rightarrow \mathrm{GFH}_{*}^{(a, b)}\left(\mathcal{H}_{F}\right)$, see Section 4.9 above. Proposition 4.37 supplies us with identifications between Floer homology of $G$ (resp., of $F$ ) with generalized Floer homology of $G$ (resp., of $F$ ). It is straightforward to check the following:

Proposition 4.38 After the identifications of GFH and $\mathrm{FH}$ the corresponding monotonicity morphisms coincide: $\operatorname{mon}_{\mathrm{FH}}=\operatorname{mon}_{\mathrm{GFH}}$. 
Moreover, the non-criticality of $a, b$ allows us to relax the condition $G>F$ to $G \geq F$.

In our calculations below we will use the following standard fact (see eg [3]):

Lemma 4.39 Let $F^{(s)}, s \in[0,1]$, be a monotone homotopy of functions constant at infinity: $F^{(s)} \leq F^{(t)}$ for $s<t$. Assume that $b$ is a non-critical value of $\mathcal{A}_{F^{(s)}}$ for all $s$. Then the natural homomorphism mon: $\mathrm{FH}^{(-\infty ; b)}\left(F^{(1)}\right) \rightarrow \mathrm{FH}^{(-\infty ; b)}\left(F^{(0)}\right)$ is an isomorphism.

4.12.2 Symplectic homology of domains Let $U \subset M$ be a bounded starshaped domain with boundary $P=\partial U$. Define the action $\operatorname{spectrum} \operatorname{spec}(P) \subset \mathbb{R}$ of $P$ as

$$
\left\{-\int_{\gamma} \alpha\right\}
$$

where $\gamma$ runs over all closed orbits of the naturally oriented characteristic foliation of $P$. Let $\mathcal{X}=\mathcal{X}(U)$ be the set of all non-positive Hamiltonian functions $F: M \rightarrow \mathbb{R}$ supported in a domain $U$. Define an "anti-natural" partial order on $\mathcal{X}$ by

$$
F \preceq G \Leftrightarrow F \geq G,
$$

and introduce symplectic homology of $U$ (see [22], [9]) as

$$
\mathrm{SH}^{(a ; b)}(U):=\underset{F \in \mathcal{X}}{\lim } \mathrm{FH}^{(a, b)}(F),
$$

where $a<b$ lie in $(-\infty ; 0) \backslash \operatorname{spec}(P)$. The grading of Floer homology induces the grading of symplectic homology by the grading set $\square^{M}$ of the Liouville manifold $(M, \omega)$. With a help of the monotonicity homomorphism in Floer homology, any inclusion $U_{1} \subset U_{2}$ gives rise to a natural grading-preserving morphism

$$
\mathrm{SH}^{\left(-\infty ; b_{1}\right)}\left(U_{1}\right) \rightarrow \mathrm{SH}^{\left(-\infty ; b_{2}\right)}\left(U_{2}\right), b_{1} \leq b_{2},
$$

of symplectic homologies. The purpose of the next sections is to extend this construction to domains in the contact manifold $(V, \operatorname{Ker}(d t-\alpha))$.

\subsection{GFH for contact forms}

In this section we focus on the contact manifold $(V, \operatorname{Ker}(d t-\alpha))$ where $V=M \times S^{1}$, as before.

Recall (see Example 4.4) that contact form $\lambda=F(d t-\alpha)$ is called admissible if $F$ equals a constant $\mathrm{C}$ at infinity and the Reeb field $R_{\lambda}$ of $\lambda$ has no contractible closed orbits of period $\leq \mathrm{C}$. 
Denote by $\mathcal{F}$ the set of all contact forms on $V$ which equal const $\cdot(d t-\alpha)$ at infinity for some positive constant, and by $\mathcal{F}_{a d} \subset \mathcal{F}$ the subset of all admissible forms. With an admissible form $\lambda$ one associates a framed Hamiltonian structure $\overrightarrow{\mathcal{H}}_{\lambda}=(\lambda)$ described in Example 4.4. Recall that the same 1-form $\lambda$ serves as its framing. The cut $\xi$ coincides with the contact structure on $V$, and the characteristic vector field is the Reeb field $R_{\lambda}$.

In what follows we will write $\mathrm{CH}^{(a, b)}(\lambda)$ instead of $\mathrm{GFH}^{(a, b)}\left(\overrightarrow{\mathcal{H}}_{\lambda}\right)$, and call this group the filtrated cylindrical contact homology of the contact form $\lambda$. We tacitly assume that $a<b<\mathrm{C}$ and that $a, b$ are non-critical values for $\mathcal{H}_{\lambda}$ (for the sake of brevity, we say that $a, b$ are non-critical values for $\lambda$ ). Note that the group $\mathrm{CH}^{(a, b)}(V, \lambda)$ is generated only by periodic orbits of $R$ which belong to classes from $\mathcal{S}$, unlike the more general situation considered in [17],[44], [48], and [4]. In particular, multiply covered periodic orbits do not contribute to this homology. As we already had seen in some applications considered above (eg, see the proof of Theorem 1.2 in Section 1.8) this is good enough for our purposes because we are able to recover the remaining part of cylindrical contact homology via a covering trick.

As in the case of Hamiltonian Floer homology, contact homology $\mathrm{CH}(\lambda)$ is graded by the grading set $\square^{M}$ of the Liouville manifold $(M, \omega)$ (see Definition 4.36 above). Indeed, denote by $\ell$ the characteristic line field of the Hamiltonian structure $\mathcal{H}_{\lambda}=(\lambda)$. Then the symplectic bundle $(T V / \ell, d \lambda)$ can be canonically identified with $(\xi, d \lambda)$. The symplectic structure on this bundle can be homotoped to $-\omega$ which corresponds to the case $\lambda=d t-\alpha$. Finally, we deform $(\xi,-\omega)$ to $(T M,-\omega)$ via the family of subbundles $\left(\xi_{s}=\operatorname{Ker}(d t-(1-s) \alpha),-\omega\right)$, where $s \in[0 ; 1]$. Furthermore, each class $e \in \mathcal{S}$ has the form $\varepsilon \oplus\left[\right.$ point $\left.\times S^{1}\right]$ with $\varepsilon \in S^{M}$. This readily yields that $\square\left(\mathcal{H}_{\lambda}\right)=\rrbracket^{M}$ (cf the beginning of the proof of Proposition 4.37 above). In the cases when either $\varepsilon$ is the class of contractible loops, or $M=T^{*} X$ and we pick any class $\varepsilon$, the contact homology are $\mathbb{Z}$-graded in accordance with Remarks 4.10 and 4.11.

If $\lambda_{+}=F \lambda_{-}$, where $\lambda_{ \pm} \in \mathcal{F}$ and the function $F$ satisfies $F>1$ (resp. $F \geq 1$ ), then we write $\lambda_{+}>\lambda_{-}$(resp. $\lambda_{+} \geq \lambda_{-}$). The forms $\lambda_{ \pm}$can be viewed as sections of the symplectization $S V=V \times \mathbb{R}_{+}$and thus if $\lambda_{+}>\lambda_{-}$, the part of the symplectization bounded by the sections $\lambda_{ \pm}$is parameterized by a directed concordance between the framed Hamiltonian structures $\overrightarrow{\mathcal{H}}_{\lambda_{-}}$and $\overrightarrow{\mathcal{H}}_{\lambda_{+}}$, see Example 4.20 above. Hence there is defined a monotonicity homomorphism

$$
\operatorname{mon}\left(\lambda_{+}, \lambda_{-}\right): \mathrm{CH}^{(a, b)}\left(\lambda_{+}\right) \rightarrow \mathrm{CH}^{(a, b)}\left(\lambda_{-}\right) .
$$

In what follows we have to deal with the case $\lambda_{+} \geq \lambda_{-}$(note the non-strict inequality). Let us make a digression to settle this situation. 
Remark 4.40 Let $\lambda$ be a contact form on $V$, and let $J$ be any almost complex structure on $\bar{V}$ adjusted to $\overrightarrow{\mathcal{H}}_{\lambda}$. Fix a real constant $c>0$. The dilation $(z, s) \rightarrow(z, c s)$ of $\bar{V}$ sends $J$ to an almost complex structure $J_{c}$ which is adjusted to $\mathcal{H}_{c \lambda}$, see Remark 4.13 above. Furthermore, each $T$-periodic orbit of the Reeb field of $\lambda$ is a $c T$-periodic orbit of the Reeb field of $c \lambda$, and hence the $c \lambda$-action differs from $\lambda$-action by the factor $c$. Therefore, we get a canonical isomorphism

$$
c_{*}: \mathrm{CH}^{(a, b)}(\lambda) \rightarrow \mathrm{CH}^{(c a, c b)}(c \lambda) .
$$

On the other hand, we have the following commutative diagram, assuming $c<1$ :

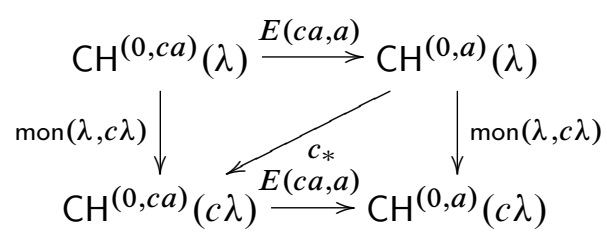

Indeed, the monotonicity homomorphism $\operatorname{mon}(\lambda, c \lambda)$ is realized by a symplectic concordance which can be endowed, after completion, by an almost complex structure which is biholomorphically equivalent to a translationally invariant almost complex structure. All index 0 holomorphic cylinders for this structure are trivial. A simple analysis of this situation shows that on the level of complexes, the homomorphisms $c_{*}$ and $\operatorname{mon}(\lambda, c \lambda)$ differ only by a varying window homomorphism.

This implies the following useful

Proposition 4.41 Suppose that $c<1, a>0$ and that the interval $[c a, a]$ does not intersect the action spectrum for the form $\lambda$. Then the monotonicity homomorphism

$$
\mathrm{CH}^{(0, a)}(\lambda) \rightarrow \mathrm{CH}^{(0, a)}(c \lambda)
$$

is an isomorphism.

This result enables us to extend the notion of monotonicity morphism between contact homology of forms $\lambda_{+}$and $\lambda_{-}$with $\lambda_{+} \geq \lambda_{-}$. In fact, we will need just the following particular case of this construction. Take any real number $a$ with $0<a<\min \left(\mathrm{C}_{-}, \mathrm{C}_{+}\right)$ which is non-critical for both $\lambda_{-}$and $\lambda_{+}$. Take $c<1$ sufficiently close to 1 so that $c \lambda_{-}<\lambda_{+}$and the interval $[c a, a]$ does not intersect the action spectrum of $\lambda_{-}$. Proposition 4.41 above provides an isomorphism

$$
\mathrm{CH}^{(0, a)}\left(\lambda_{-}\right) \rightarrow \mathrm{CH}^{(0, a)}\left(c \lambda_{-}\right) .
$$


Its composition with the (already defined) monotonicity morphism

$$
\mathrm{CH}^{(0, a)}\left(\lambda_{+}\right) \rightarrow \mathrm{CH}^{(0, a)}\left(c \lambda_{-}\right)
$$

gives the desired monotonicity morphism

$$
\operatorname{mon}\left(\lambda_{+}, \lambda_{-}\right): \mathrm{CH}^{(0, a)}\left(\lambda_{+}\right) \rightarrow \mathrm{CH}^{(0, a)}\left(\lambda_{-}\right) .
$$

It does not depend on the particular choice of the constant $c$.

Given a compactly supported contactomorphism $\Phi$ and a contact form $\lambda$, we write $\Phi_{*} \lambda$ for $\left(\Phi^{*}\right)^{-1} \lambda$ and $\Phi_{\sharp}$ for the induced isomorphism $\mathrm{CH}(\lambda) \rightarrow \mathrm{CH}\left(\Phi_{*} \lambda\right)$.

Proposition 4.42 Suppose we are given two contact forms $\lambda_{+}, \lambda_{-}$such that $\lambda_{+} \geq \lambda_{-}$. Let $\Phi^{t}: V \rightarrow V, t \in[0,1]$, be a compactly supported contact isotopy with $\Phi^{0}=\mathbf{1}$ and such that for all $t \in[0,1]$ we have $\Phi_{*}^{t} \lambda_{+} \geq \lambda_{-}$. Then the following diagram commutes:

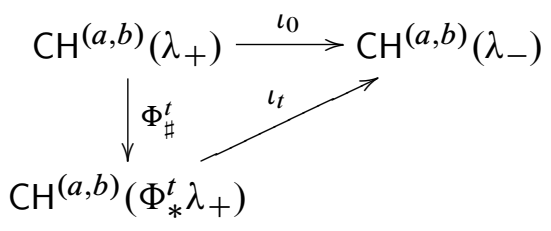

where $\iota_{t}=\operatorname{mon}\left(\Phi_{*}^{t} \lambda_{+}, \lambda_{-}\right)$.

Proof Put $\lambda_{+}^{t}=\Phi_{*}^{t} \lambda_{+}$. It suffices to prove the proposition for the case when $\lambda_{+}^{t}>\lambda_{-}$ for all $t$. Write $\Pi$ for the contact form $s(d t-\alpha)$ on the symplectization $S V$ and denote $W=V \times[0 ; 1]$. Let $\varphi_{t}: W \rightarrow S V$ be the embedding given by formula (52) of Example 4.20 above, so that $\left(W,\left(\varphi_{t}^{*} \Pi\right)\right)$ is a directed concordance between Hamiltonian structures $\mathcal{H}_{\lambda_{+}^{t}}$ and $\mathcal{H}_{\lambda_{-}}$. Note that the image of $\varphi_{t}$ is the domain cut out from the symplectization $S V$ by the graphs of the forms $\lambda_{+}^{t}$ and $\lambda_{-}$. The isotopy $\Phi^{t}$ lifts to a family of $\mathbb{R}_{+}$-equivariant symplectomorphisms $\widetilde{\Phi}^{t}$ of $S V$. Cutting off the Hamiltonian which generates $\tilde{\Phi}^{t}$ outside a neighborhood of $\operatorname{graph}\left(\lambda_{+}^{t}\right)$ we get a family of symplectomorphisms $\Psi^{t}$ which send Image $\left(\varphi_{0}\right)$ onto Image $\left(\varphi_{t}\right)$, coincide with $\tilde{\Phi}^{t}$ near $\operatorname{graph}\left(\lambda_{+}\right)$and are equal to the identity near $\operatorname{graph}\left(\lambda_{-}\right)$. Define a diffeomorphism $A: W \rightarrow W$ by

$$
A=\varphi_{t}^{-1} \circ \Psi_{t} \circ \varphi_{0} .
$$

Note that the restriction of $A$ to $V \times\{1\}$ sends the Hamiltonian structure $\mathcal{H}_{\lambda_{+}}$to $\mathcal{H}_{\lambda_{+}^{t}}$, while the restriction of $A$ to $V \times\{0\}$ is the identity map. Take now the concordances $\left(W,\left(\varphi_{0}^{*} \Pi\right)\right)$ and $\left(W,\left(\varphi_{t}^{*} \Pi\right)\right)$. The proposition follows from commutative diagram (59). 


\subsection{Contact homology of domains}

Let $U \subset V$ be an open subset with compact closure, and let $\lambda \in \mathcal{F}_{a d}$ be an admissible form. We denote by $\mathcal{F}(U, \lambda)$ the set of contact forms which coincide with $\lambda$ outside of $U$, and by $\mathcal{F}_{a d}(U, \lambda)$ its subset consisting of admissible forms. The set $\mathcal{F}(U, \lambda)$ is endowed with the "anti-natural" partial order $\preceq$ defined as follows:

$$
\lambda^{\prime \prime} \preceq \lambda^{\prime} \Leftrightarrow \lambda^{\prime \prime} \geq \lambda^{\prime} .
$$

An increasing sequence in a partially ordered set is called dominating if every element of the set is $\preceq$ than some element of the sequence.

Definition 4.43 An admissible form $\lambda$ on $V$ is called $U$-basic if the partially ordered set $\mathcal{F}(U, \lambda)$ admits a dominating sequence consisting of admissible forms. Domains $U$ admitting a basic form are called admissible.

This means, in the down to earth language, that there exists a sequence of admissible forms $\lambda_{i} \in \mathcal{F}_{a d}(U, \lambda)$ such that $\lambda_{i} / \lambda$ converges to zero on every compact subset of $U$ as $i \rightarrow \infty$.

Let $\lambda$ be a $U$-basic form of an admissible domain $U$. For $\varepsilon>0$ denote by $\mathcal{F}_{a d}(U, \lambda, \varepsilon)$ the set of all forms from $\mathcal{F}_{a d}(U, \lambda)$ which do not have $\varepsilon$ as a critical value. Given a dominating sequence in $\mathcal{F}_{a d}(U, \lambda)$, one can perturb it to a dominating sequence in $\mathcal{F}_{a d}(U, \lambda, \varepsilon)$ provided $\varepsilon$ is small enough. Define contact homology of $U$ as follows:

$$
\mathrm{CH}(U \mid \lambda)=:=\lim _{\varepsilon \rightarrow 0} \underset{\lambda^{\prime} \in \mathcal{F}_{a d}(U, \lambda, \varepsilon)}{\underset{\lim _{\longrightarrow}}{\longrightarrow}} \mathrm{CH}^{(0, \varepsilon)}\left(\lambda^{\prime}\right) .
$$

Let $\lambda_{i} \in \mathcal{F}_{a d}(U, \lambda)$ be a dominating sequence. Unveiling the definitions of direct and inverse limits we see (after, possibly, a small perturbation of $\left\{\lambda_{i}\right\}$ ) that every element of $\mathrm{CH}(U \mid \lambda)$ can be represented as a sequence

$$
\left\{x_{N}\right\}, \quad x_{N} \in \mathrm{CH}^{\left(0 ; \frac{1}{N}\right)}\left(\lambda_{i_{N}}\right), \quad i_{N} \rightarrow \infty,
$$

such that for $K>N$ the images of $x_{N}$ and $x_{K}$ under the natural morphisms coincide in $\mathrm{CH}^{\left(0 ; \frac{1}{N}\right)}\left(\lambda_{j}\right)$ for some $j \geq \max \left(i_{N}, i_{K}\right)$.

The grading of contact homology of forms induces the grading of contact homology of domains by elements of the grading set $\rrbracket^{M}$ of the Liouville manifold (see Definition 4.36).

Clearly, the notions introduced above are invariant under the action of the group $\mathcal{G}$ of all compactly supported contactomorphisms of $V$. We sum up this in the following lemma. 
Lemma 4.44 Let $U$ be an admissible domain with a basic form $\lambda$. Let $\Phi \in \mathcal{G}$ be a contactomorphism. Then the domain $\tilde{U}=\Phi(U)$ is admissible with a basic form $\tilde{\lambda}:=\Phi_{*} \lambda$. Moreover, there is a canonical isomorphism

$$
\Phi_{\sharp}: \mathrm{CH}(U \mid \lambda) \rightarrow \mathrm{CH}(\tilde{U} \mid \tilde{\lambda}) .
$$

Proof One can define $\mathrm{CH}(U)$ starting from the $U$-basic form $\lambda$, and define $\mathrm{CH}(\Phi(U))$ starting from the basic form $\tilde{\lambda}=\Phi_{*} \lambda$. A dominating sequence $\lambda_{i} \in \mathcal{F}_{a d}(U, \lambda)$ is mapped by $\Phi_{*}$ to a dominating sequence $\tilde{\lambda}_{i} \in \mathcal{F}_{a d}(\Phi(U), \tilde{\lambda})$. On the other hand, for any contact form $\lambda_{i}$ the contactomorphism $\Phi$ induces an isomorphism $\Phi_{\sharp}: \mathrm{CH}^{(0, \varepsilon)}\left(\lambda_{i}\right) \rightarrow \mathrm{CH}^{(0, \varepsilon)}\left(\tilde{\lambda}_{i}\right)$. These isomorphisms commute with the monotonicity morphisms mon $i j: \mathrm{CH}^{(0, \varepsilon)}\left(\lambda_{i}\right) \rightarrow \mathrm{CH}^{(0, \varepsilon)}\left(\lambda_{j}\right)$ and $\widetilde{\operatorname{mon}}_{i j}: \mathrm{CH}^{(0, \varepsilon)}\left(\tilde{\lambda}_{i}\right) \rightarrow \mathrm{CH}^{(0, \varepsilon)}\left(\tilde{\lambda}_{j}\right)$, for $i<j$. Hence $\Phi_{\sharp}$ descends to the required isomorphism

$$
\Phi_{\sharp}: \mathrm{CH}(U \mid \lambda) \rightarrow \mathrm{CH}(\tilde{U} \mid \tilde{\lambda})
$$

between the limits.

An important example of admissible domains is given by the next lemma.

Lemma 4.45 Let $U \subset V$ be a fiberwise starshaped domain. Then the form $d t-\alpha$ is $U$-basic. In particular, $U$ is admissible.

Proof We claim that an admissible form $\lambda_{0}=d t-\alpha$ is $U$-basic. Indeed, put $P_{t}=\partial U \cap(M \times\{t\})$. Let us recall that the complement of Core $(M)$ can be identified with the symplectization $S P_{t}$, and the Liouville form $\alpha$ can be written as $u \beta_{t}$ for the contact form $\beta_{t}=\left.\alpha\right|_{T P_{t}}$. In these coordinates we have $\lambda_{0}=d t-u \beta_{t}$. Take a sequence $G_{i}$ of non-decreasing positive functions $G_{i}: \mathbb{R}_{+} \rightarrow \mathbb{R}$ which are constant near 0 , equal 1 for $u>1$ and such that $G_{i}$ uniformly converge to 0 on every compact subset of $U$. Clearly, the sequence $\lambda_{i}:=G_{i} \lambda_{0} \in \mathcal{F}\left(U, \lambda_{0}\right)$ is dominating. To see that the forms $\lambda_{i}$ are admissible note that on $(M \backslash \operatorname{Core}(M)) \times S^{1}$ we have

$$
\left.d \lambda_{i}\right|_{M \times\{t\}}=-d\left(G_{i} u\right) \wedge \beta_{t}-G_{i} u d \beta_{t} .
$$

Since $\frac{d\left(G_{i} u\right)}{d u}>0$ we conclude that the restriction of $d \lambda_{i}$ to the fibers $M \times\{t\}$ is non-degenerate. Hence the Reeb field of $\lambda_{i}$ is transversal to the fibers $M \times\{t\}$, and therefore it has no contractible closed orbits. This proves that all $\lambda_{i}$ are admissible.

Proposition 4.46 Let $U \subset V$ be an admissible domain. With any two $U$-basic forms $\lambda$ and $\lambda^{\prime}$ one can associate an isomorphism

$$
q\left(\lambda^{\prime}, \lambda\right): \mathrm{CH}\left(U \mid \lambda^{\prime}\right) \rightarrow \mathrm{CH}(U \mid \lambda) .
$$


This correspondence is functorial in the sense that for any three $U$-basic forms $\lambda_{1}, \lambda_{2}$ and $\lambda_{3}$ we have

$$
q\left(\lambda_{1}, \lambda_{3}\right)=q\left(\lambda_{2}, \lambda_{3}\right) \circ q\left(\lambda_{1}, \lambda_{2}\right)
$$

\section{Proof}

Step 1 Let $\lambda^{\prime}$ and $\lambda$ be two $U$-basic forms such that $\lambda^{\prime}>\lambda$. Denote by $\left\{\lambda_{i}^{\prime}\right\}$ and $\left\{\lambda_{i}\right\}$ the corresponding dominating sequences of admissible forms. We claim that for every $i$ there exists $j$ such that $\lambda_{i}^{\prime}>\lambda_{j}$. Indeed, there exists a compact $K \subset U$ such that $\lambda_{i}^{\prime}>\lambda_{1}$ on $V \backslash K$. Thus $\lambda_{i}^{\prime}>\lambda_{j}$ on $V \backslash K$ for all $j$ since $\lambda_{1} \geq \lambda_{j}$. Choose now $j$ so large that $\lambda_{i}^{\prime}>\lambda_{j}$ on $K$. The claim follows.

It follows from the claim that the dominating admissible sequences $\lambda_{i}^{\prime} \in \mathcal{F}_{a d}\left(U, \lambda^{\prime}\right)$ and $\lambda_{i} \in \mathcal{F}_{a d}(U, \lambda)$ can be chosen in such a way that for all $i$ we have $\lambda_{i}^{\prime}>\lambda_{i}$. By passing to double limits in the family of monotonicity homomorphisms

$$
\operatorname{mon}\left(\lambda_{i}^{\prime}, \lambda_{i}\right): \mathrm{CH}^{(0, \varepsilon)}\left(\lambda_{i}^{\prime}\right) \rightarrow \mathrm{CH}^{(0, \varepsilon)}\left(\lambda_{i}\right)
$$

we get a homomorphism $\mathrm{CH}\left(U \mid \lambda^{\prime}\right) \rightarrow \mathrm{CH}(U \mid \lambda)$ which will be denoted by $q\left(\lambda^{\prime}, \lambda\right)$. Then, given three forms $\lambda_{1}, \lambda_{2}, \lambda_{3}$ with $\lambda_{i}>\lambda_{j}$ for $i>j$ the formula

$$
q\left(\lambda_{1}, \lambda_{3}\right)=q\left(\lambda_{2}, \lambda_{3}\right) \circ q\left(\lambda_{1}, \lambda_{2}\right)
$$

follows from the corresponding property for the monotonicity homomorphisms (see Theorem 4.29 above).

Step 2 Next, we claim that $q\left(\lambda_{0}, c \lambda_{0}\right)$ is an isomorphism for any $U$-basic form $\lambda_{0}$ and any $c \in(0,1)$. Note that if $\lambda \in \mathcal{F}\left(U, \lambda_{0}\right)$ then $c \lambda \in \mathcal{F}\left(U, c \lambda_{0}\right)$. Look at commutative diagram (62): First, pass to the direct limit as $\lambda \in \mathcal{F}\left(U, \lambda_{0}\right)$. Then consider the inverse limit as $a \rightarrow 0$. The horizontal arrows become the identity maps, so the vertical and the diagonal arrows of the diagram coincide in the double limit. Note that the vertical arrows correspond to the homomorphism $q\left(\lambda_{0}, c \lambda_{0}\right)$. The claim follows from the fact that the diagonal arrow $c_{*}$ in the diagram is an isomorphism for all $a$ and $\lambda$.

Step 3 Now for any two forms $\lambda, \lambda^{\prime}$ such that $\lambda^{\prime}>\lambda$ we have for a sufficiently small constant $c>0$ :

$$
\frac{1}{c} \lambda>\lambda^{\prime}>\lambda>c \lambda^{\prime},
$$

and hence we have

$$
q\left(\lambda^{\prime}, \lambda\right) \circ q\left(\frac{1}{c} \lambda, \lambda^{\prime}\right)=q\left(\frac{1}{c} \lambda, \lambda\right)
$$


and

$$
q\left(\lambda, c \lambda^{\prime}\right) \circ q\left(\lambda^{\prime}, \lambda\right)=q\left(\lambda^{\prime}, c \lambda^{\prime}\right)
$$

which implies that $q\left(\lambda^{\prime}, \lambda\right)$ is an isomorphism. Finally for any two $U$-basic forms $\lambda_{0}$ and $\lambda_{1}$ choose a $U$-basic form $\lambda$ such that $\lambda<\lambda_{0}$ and $\lambda<\lambda_{1}$ and put

$$
q\left(\lambda_{0}, \lambda_{1}\right)=q\left(\lambda_{1}, \lambda\right)^{-1} \circ q\left(\lambda_{0}, \lambda\right) .
$$

It is straightforward to check that the definition is independent of $\lambda$ and satisfies the required functoriality condition.

Therefore, from now on we can omit the $U$-basic form $\lambda$ from the notation and write

$$
\mathrm{CH}(U)=\mathrm{CH}(U \mid \lambda) \text {. }
$$

The monotonicity homomorphism for contact homology of forms induces an inclusion homomorphism in $\left(U^{\prime}, U\right): \mathrm{CH}\left(U^{\prime}\right) \rightarrow \mathrm{CH}(U)$ for admissible domains $U, U^{\prime}$ with $U^{\prime} \subset U$. Indeed, we can choose dominating sequences of forms $\left\{\lambda_{k}\right\}$ for $U$ and $\left\{\lambda_{k}^{\prime}\right\}$ for $U^{\prime}$ so that $\lambda_{k}^{\prime} \geq \lambda_{k}$. The monotonicity maps $\operatorname{mon}_{k}: \mathrm{CH}^{(0 ; \varepsilon)}\left(\lambda_{k}^{\prime}\right) \rightarrow \mathrm{CH}^{(0 ; \varepsilon)}\left(\lambda_{k}\right)$ induce the inclusion homomorphism in the double limit when $k \rightarrow \infty$ and $\varepsilon \rightarrow 0$.

On the other hand, according to Lemma 4.44 a contactomorphism $\Phi \in \mathcal{G}$ induces an isomorphism $\Phi_{\sharp}: \mathrm{CH}(U) \rightarrow \mathrm{CH}(\Phi(U))$.

It is easy to see that contact homology $\mathrm{CH}(U)$ of admissible domains, inclusion morphisms in $\left(U^{\prime}, U\right)$ and induced isomorphisms $\Phi_{\#}$ satisfy conditions (5)-(8) of Section 1.8 of the introduction. We summarize this in the following theorem in the language of category theory. In order to avoid cumbersome formulations we will assume that compactly supported contactomorphisms of $V$ induce grading-preserving morphisms of contact homology. This happens, for instance, when $M$ is simply connected, when $\operatorname{dim} M \geq 4$ and $M$ is Weinstein, or when $\operatorname{dim} M \geq 6$. In the latter two cases we have, maybe after a perturbation of the Liouville structure, that $\operatorname{codim}(\operatorname{Core}(M)) \geq 2$, and hence every free homotopy class of loops in $M$ has a representative outside an arbitrarily large compact subset.

Theorem 4.47 Assume that either $M$ is simply connected, or $\operatorname{dim} M \geq 4$ and $M$ is Weinstein, or $\operatorname{dim} M \geq 6$. Consider a category whose objects are admissible open domains of $V$ and morphisms correspond to inclusions. Then the correspondence $U \rightarrow \mathrm{CH}(U)$ is a $\mathcal{G}$-functor of this category to the category of $\rrbracket^{M}$-graded vector spaces over $\mathbb{Z}_{2}$, where $\mathcal{G}$ is the group of compactly supported contactomorphisms of $V$ and $\rrbracket^{M}$ is the grading set of the Liouville manifold $(M, \omega)$. 
Since fiberwise starshaped domains are admissible, we recover results stated in the beginning of Section 1.8 of the introduction.

In the remaining cases, eg, when $M$ is a non-contractible Liouville surface, the same result holds true if either we replace the group $\mathcal{G}$ by its identity component, or restrict ourselves to contact homology in the class of contractible loops. In the general case the categorical formulation is more clumsy and is omitted.

The next theorem and its corollary is not used in the sequel, but we present them for the completeness of the picture.

Theorem 4.48 Let $U$ be an admissible domain, and $\Phi \in \mathcal{G}$ a contactomorphism which admits an isotopy $\Phi^{t}$ connecting $\Phi^{1}=\Phi$ with $\Phi^{0}=\mathbf{1}$ such that $\operatorname{Supp} \Phi^{t} \subset U$ for all $t \in[0,1]$. Then for any admissible sub-domain $U^{\prime} \subset U$ the following diagram commutes:

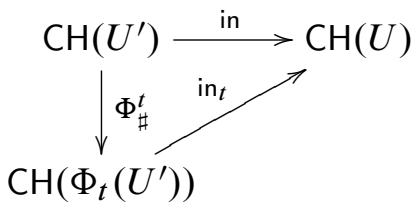

where in $=\operatorname{in}\left(U^{\prime}, U\right)$ and $\operatorname{in}_{t}=\operatorname{in}\left(\Phi^{t}\left(U^{\prime}\right), U\right)$.

Proof We can choose dominating sequences of forms $\lambda_{k}$ for $U$ and $\lambda_{k}^{\prime}$ for $U^{\prime}$ such that for all $t \in[0,1]$ we have $\Phi_{*}^{t} \lambda_{k}^{\prime} \geq \lambda_{k}$. Then according to Proposition 4.42 we have a commutative diagram

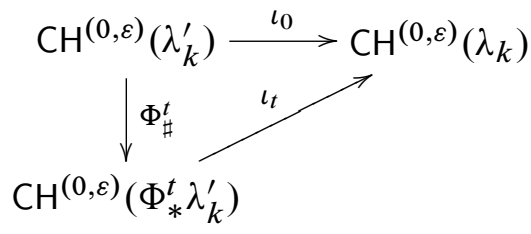

where $\iota_{t}=\operatorname{mon}\left(\Phi_{*}^{t} \lambda_{k}^{\prime}, \lambda_{k}\right)$ is the monotonicity homomorphism. Passing to the double limit when $k \rightarrow \infty$ and $\varepsilon \rightarrow 0$ we get the diagram (64).

Taking $U^{\prime}=U$ in this theorem, we get the following result.

Corollary 4.49 Assume that a contactomorphism $\Phi \in \mathcal{G}$ is isotopic to the identity through contactomorphisms supported in an admissible domain $U$. Then the induced isomorphism $\Phi_{\sharp}: \mathrm{CH}(U) \rightarrow \mathrm{CH}(U)$ is the identity map. 


\section{Calculations with contact homology}

Here we complete the proof of several "hard" results stated in Section 1. First of all we express contact homology of the prequantization of a symplectic domain in terms of its filtrated symplectic homology, see Theorem 5.3 and Proposition 5.6 below which together form a slightly more explicit version of Theorem 1.27. Our approach is based on generalized Floer homology theory developed in Section 4. We apply this result to calculations with contact homology. In particular, we prove Theorems 1.28 and 1.29 on contact homology of prequantizations of ellipsoids and balls in $\mathbb{R}^{2 n}$. Furthermore, we study contact homology of prequantizations of unit ball bundles of closed manifolds in terms of cohomology of free loop spaces. As a result, we prove Theorem 1.18 on orderability of spaces of contact elements.

\subsection{Contact homology of split domains}

Let $\left(M^{2 n}, \omega, L\right)$ be a Liouville manifold with the Liouville form $\alpha=i_{L} \omega$. We will work on the manifold $V=M \times S^{1}$, write $t(\bmod 1)$ for the coordinate on $S^{1}=\mathbb{R} / \mathbb{Z}$, and orient $V$ by the volume form $(-\omega)^{n} \wedge d t$. Consider the contact structure $\xi=\operatorname{Ker}(d t-\alpha)$ on $V$. Recall that the notion of contact characteristic foliation of a hypersurface in a contact manifold was defined in Section 1.3.

Definition 5.1 A hypersurface $\Sigma$ with smooth boundary in a contact manifold is called non-resonant if it is transversal to the contact structure and the contact characteristic foliation of $\Sigma$ has no closed orbits.

Example 5.2 Let $P \subset \mathbb{R}^{2 n}$ be any starshaped hypersurface. Then the hypersurface $\widehat{P}=P \times S^{1}$ is non-resonant if and only if the symplectic characteristic foliation on $P$ (equipped with the natural orientation) has no closed orbits $\gamma$ with $\int_{\gamma} \alpha=1$. With the notation of Section 4.12.2 this condition means that $-1 \notin \operatorname{spec}(P)$.

Theorem 5.3 Let $U \subset M$ be an open domain with compact closure and smooth starshaped boundary $P=\partial U$. Suppose that the hypersurface $P \times S^{1}$ is non-resonant. Then there exists a grading-preserving isomorphism

$$
\Psi_{U}: \mathrm{CH}\left(U \times S^{1}\right) \rightarrow \mathrm{SH}^{(-\infty ;-1)}(U) .
$$

Note that both contact and symplectic homologies in this theorem are graded by the grading set $\rrbracket^{M}$ of the Liouville manifold $(M, \omega)$. 
Proof Recall that we have the canonical decomposition $M=S P \sqcup \operatorname{Core}(M)$ (see formula (2) of Section 1.5). Consider the contact form $\beta=\left.\alpha\right|_{T P}$ on $P$ and fix the identification $S P=P \times \mathbb{R}_{+}$associated to $\beta$ (see Section 1.3 above). We use coordinates $(x, u) \in P \times \mathbb{R}_{+}$on $S P$ and extend $u$ by 0 to $\operatorname{Core}(M)$. With this notation we have $U=\{u<1\}$.

\section{Step I From contact to Floer homology}

Denote by $\mathcal{H}$ the set of continuous Hamiltonians $H: M \rightarrow(0,+\infty)$ with the following properties:

- On $S P=P \times(0 ;+\infty)$ the Hamiltonian $H$ is a piecewise smooth function of one variable $u$, that is $H=H(u)$;

- $H(u) \equiv c$ for $u \in(0, a)$ and $H(u) \equiv 1$ for $u \geq b$, where $c>0$ and $0<a<b<1$ are some constants depending on $H$;

- At each smooth point $u \in(0,+\infty)$,

$$
H(u)-u H^{\prime}(u)>0,
$$

where $H^{\prime}$ stands for the derivative of $H$. Note that the expression in the left hand side of this inequality can be interpreted as the vertical coordinate of the intersection point between the tangent line to $\operatorname{graph}(H)$ and the vertical axis;

- $H \equiv c$ on $\operatorname{Core}(M)=M \backslash S P$.

Given $H \in \mathcal{H}$ as above, define a function $\phi_{H}: \mathbb{R}_{+} \rightarrow \mathbb{R}_{+}$by

$$
\phi_{H}(u)=\frac{u}{H(u)} .
$$

One readily checks that $\phi_{H}$ is a homeomorphism of $\mathbb{R}_{+}$whose inverse can be written as $\phi_{\bar{H}}$ for some function $\bar{H} \in \mathcal{H}$. Moreover, $\phi_{H}$ is a diffeomorphism when $H$ is smooth. Define a map $\Phi_{H}: V \rightarrow V$ as follows: put $\Phi_{H}(x, u, t)=\left(x, \phi_{H}(u), t\right)$ on $P \times(0,+\infty) \times S^{1}$ and extend it by $(z, t) \rightarrow\left(L^{-\log c} z, t\right)$ to Core $(M) \times S^{1}$. Again, $\Phi_{H}$ is a homeomorphism of $V$ with $\Phi_{H}^{-1}=\Phi_{\bar{H}}$ and $\Phi_{H}$ is a diffeomorphism when $H$ is smooth.

Put $\lambda_{H}:=\frac{d t-\alpha}{H}$ and $\sigma_{H}:=\bar{H} d t-\alpha$. The key property of $\Phi_{H}$ is given by the following

Lemma 5.4 $\Phi_{H}^{*} \lambda_{H}=\sigma_{H}$ for every smooth $H \in \mathcal{H}$. 
We deduce from the lemma that for every $\epsilon \in(0,1)$

$$
\mathrm{CH}^{(0 ; \epsilon)}\left(\lambda_{H}\right)=\mathrm{CH}^{(0 ; \epsilon)}\left(\sigma_{H}\right)=\mathrm{GFH}^{(0 ; \epsilon)}\left(V,\left(\sigma_{H}\right)\right)=\mathrm{FH}^{(0, \epsilon)}(\bar{H}),
$$

where the right equality follows from Proposition 4.37. Furthermore, these identifications preserve the grading since the diffeomorphism $\Phi_{H}$ is isotopic to the identity. Note that $\lambda_{H}$ is a contact form corresponding to the contact structure $\xi$. Hence equation (66) links together contact homology and Hamiltonian Floer homology. Let us emphasize that the main purpose of generalized Floer homology theory developed in Section 4 is to justify this link. Eventually, this link will enable us to complete the proof of the theorem.

Proof of Lemma 5.4 Clearly it suffices to verify the statement of the lemma on $S P \times S^{1}$. Recall that with respect to the splitting $S P=P \times(0,+\infty)$ the Liouville form $\alpha$ can be written as $u \beta$ where $\beta=\left.\alpha\right|_{T P}$. Put $v=\phi_{H}(u)$ and observe that

$$
\frac{d t-u \beta}{H(u)}=\frac{d t}{H\left(\phi_{\bar{H}}(v)\right)}-v \beta \text {. }
$$

On the other hand,

which yields

$$
\frac{\phi_{\bar{H}}(v)}{H\left(\phi_{\bar{H}}(v)\right)}=v
$$

$$
H\left(\phi_{\bar{H}}(v)\right)=\frac{\phi_{\bar{H}}(v)}{v}=\frac{1}{\bar{H}(v)}
$$

Therefore,

$$
\frac{d t-u \beta}{H(u)}=\bar{H}(v) d t-v \beta,
$$

as required.

One readily checks that the transform $H \rightarrow \bar{H}$ is continuous in the uniform topology and anti-monotone:

$$
H_{1} \leq H_{2} \Rightarrow \bar{H}_{1} \geq \bar{H}_{2} .
$$

Moreover, this transform preserves the class of piecewise linear functions from $\mathcal{H}$. We will work out this property for the following special class of functions. For $0<a<b<1$ and $c>1$ consider a piecewise linear function $F_{a, b, c} \in \mathcal{H}$ which equals $c$ on $(0, a)$, equals 1 on $(b,+\infty)$ and which is linear on $[a, b]$ (see Figure 1). A straightforward calculation shows that

$$
\bar{F}_{a, b, c}=F_{a / c, b, 1 / c} .
$$




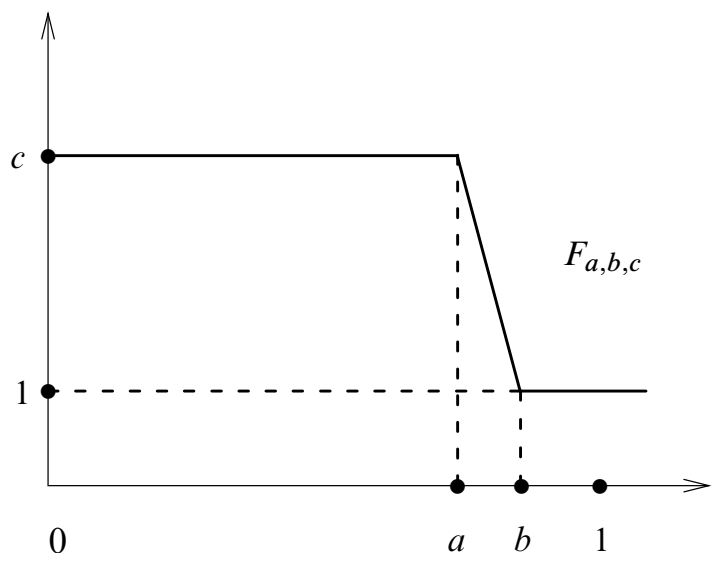

Figure 1

For $0<\mu<v<1$ and $\kappa<0$ let us consider a piecewise linear function $G_{\mu, v, \kappa}$ which equals $\kappa$ on $[0, \mu]$, equals 0 on $[\nu,+\infty]$ and which is linear on $[\mu, \nu]$. With this notation

$$
\bar{F}_{a, b, c}-1=G_{a / c, b,-1+1 / c},
$$

see Figure 2.

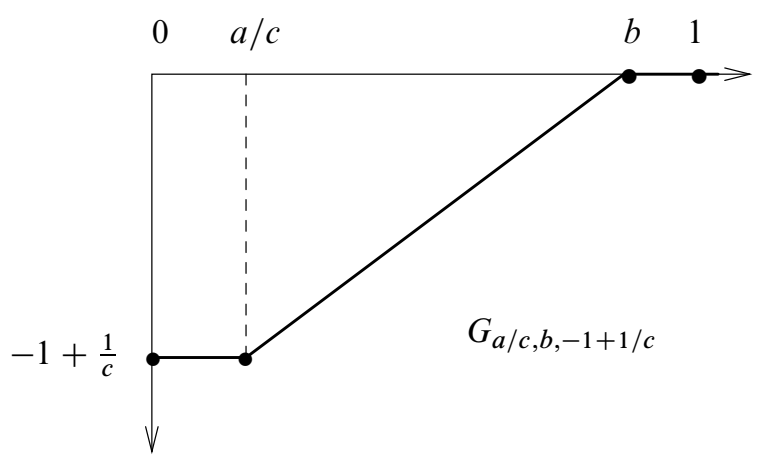

Figure 2

Note that $G_{a / c, b,-1+1 / c}$ is a non-positive Hamiltonian supported in $U$. Such Hamiltonians appear in the definition of symplectic homology of $U$ presented in Section 4.12.2. A troubling point, of course, is that the function $G_{a / c, b,-1+1 / c}$ is not smooth. We are going to take care of this right now.

\section{Step II A smoothing procedure}


In what follows we are going to work with Floer homology of Hamiltonians $G_{\mu, v, \kappa}$, which is understood as Floer homology of a smooth function which approximates $G_{\mu, \nu, \kappa}$ in the uniform topology. A careful choice of the smooth approximations will enable us to perform precise homological calculations. Let us explain the details of the approximation procedure we use.

Let $\mathcal{L}:=P T \mathbb{R}^{2}$ be the projectivization of the tangent bundle of the plane $\mathbb{R}^{2}$. Define the set of generalized tangent lines $\mathcal{T}_{\mu, v, \kappa} \subset \mathcal{L}$ to graph $\left(G_{\mu, v, \kappa}\right)$ as follows:

$$
\mathcal{T}_{\mu, v, \kappa}=\left\{\left(l,\left(u, G_{\mu, v, \kappa}(u)\right)\right\}\right.
$$

so that

- $l$ is the usual tangent line for $u \notin\{\mu, \nu\}$;

- $l$ lies above $\operatorname{graph}\left(G_{\mu, v, \kappa}\right)$ for $u=v$;

- $l$ lies below $\operatorname{graph}\left(G_{\mu, v, \kappa}\right)$ for $u=\mu$.

The Euclidean metric on $\mathbb{R}^{2}$ gives rise to a natural metric on $\mathcal{L}$. The proof of the next elementary lemma is straightforward.

Lemma 5.5 For a small enough $\delta>0$ there exists a smooth function $H=H_{\mu, v, \kappa}$ on $[0,+\infty)$ with the following properties (see Figure 3 ):

- $H(0)=\kappa, H^{\prime}(0)=0$;

- $H(u)=0$ for all $u \geq 1$;

- $H(u)<0, H^{\prime}(u)>0, H(u)-u H^{\prime}(u)<0$ for all $u \in(0,1)$;

- The tangent bundle $T(\operatorname{graph}(H))$, considered as a subset of $\mathcal{L}$, lies in the $\delta$ neighborhood of $\mathcal{T}_{\mu, v, \kappa}$.

Moreover, the $\delta$-approximation $H_{\mu, v, \kappa}$ can be chosen to depend continuously on the parameters $\mu, v, \kappa, \delta$.

\section{Step III Homological calculations}

Take $\epsilon>0$ small enough. Choose $a, b, c, \delta$ depending on $\epsilon$ so that $a$ and $b$ are sufficiently close to $1, c$ is large enough and $\delta$ is small enough. We assume that

$$
\epsilon>\frac{2}{c} \text {. }
$$

Let $H$ be a $\delta$-approximation of $G_{a / c, b,-1+1 / c}$, as in Lemma 5.5. The goal of this Step is to establish the following formula:

$$
\mathrm{FH}^{(-1 ;-1+\epsilon)}(H)=\mathrm{FH}^{(-\infty,-1+\epsilon)}(H)=\mathrm{SH}^{(-\infty ;-1+\epsilon)}(U) .
$$




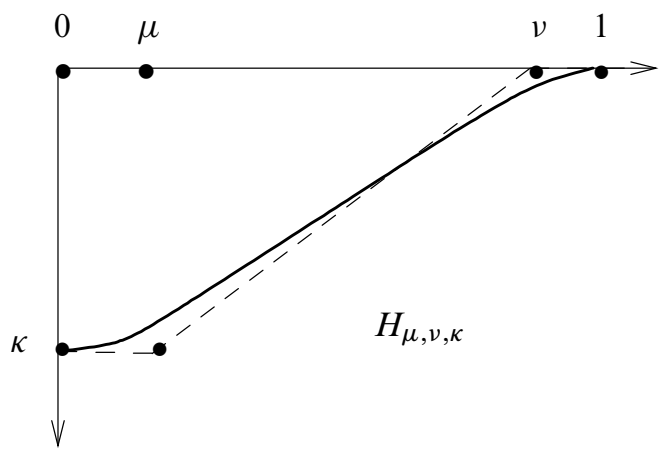

Figure 3

To start with, let us recall (see equation (20) above) that the Hamiltonian vector field of the Hamiltonian $H=H(u)$ is given by $H^{\prime}(u) R$ on $S P=P \times(0,+\infty)$ and it vanishes outside this domain, where $R$ stands for the Reeb vector field of $\beta$. Hence its 1-periodic orbits are either the fixed points lying in $M \backslash(P \times(0,1))$, or the pairs $(\gamma, u)$ where $u \in(0,1)$ and $\gamma$ is a closed orbit of the Reeb field $R$ of period $H^{\prime}(u)$. In the latter case $-H^{\prime}(u) \in \operatorname{spec}(P)$. The symplectic action of an orbit corresponding to a fixed point is simply the value of the Hamiltonian at this point. The symplectic action of an orbit of the type $(\gamma, u)$ equals $H(u)-u H^{\prime}(u)$. Recall that this quantity can be interpreted as the vertical coordinate of the intersection point between the tangent line to $\operatorname{graph}(H)$ and the vertical axis.

Since $P \times S^{1}$ is assumed to be non-resonant, there exists $\tau>0$ such that

$$
\operatorname{spec}(P) \cap(-1-\tau,-1+\tau)=\varnothing .
$$

Our next claim is that the actions of all 1-periodic orbits of $H$ are greater than -1 . Indeed, look at Figure 2. The claim will readily follow from the fact that any line from $\mathcal{T}_{a / c, b,-1+1 / c}$ intersects the vertical axis above -1 . Clearly, it suffices to check this for the line which passes through $(b, 0)$ and $(a / c,-1+1 / c)$. Calculating, we get that it intersects the vertical axis at a point $(0, y)$ with

$$
y=-\frac{b c-b}{b c-a} .
$$

Since $a<b$ we have $y>-1$ as required, and the claim follows.

The claim yields

$$
\mathrm{FH}^{(-1,-1+\epsilon)}(H)=\mathrm{FH}^{(-\infty,-1+\epsilon)}(H),
$$

which proves the left equality in formula (72). To show that

$$
\mathrm{FH}^{(-\infty,-1+\epsilon)}(H)=\mathrm{SH}^{(-\infty,-1+\epsilon)}(U)
$$


it suffices to produce a monotone homotopy $H^{(s)}, s \geq 0$, such that $H^{(0)}=H$, the family $H^{(s)}$ is dominating with respect to "anti-natural" order $\preceq$ on $\mathcal{F}$, and, crucially, for every $s$ the point $-1+\epsilon$ does not lie in the action spectrum of $H^{(s)}$ (see Lemma 4.39).

To construct $H^{(s)}$, we start with a monotone homotopy $G^{(s)}=G_{\mu(s), \nu(s), \kappa(s)}, s \geq 0$, of the function $G^{(0)}=G_{a / c, b,-1+1 / c}$ through a dominating family, see Figure 4. Next, we take $H^{(s)}$ as a smooth $\delta(s)$-approximation of $G^{(s)}$ provided by Lemma 5.5 with $\delta(s)$ small enough.

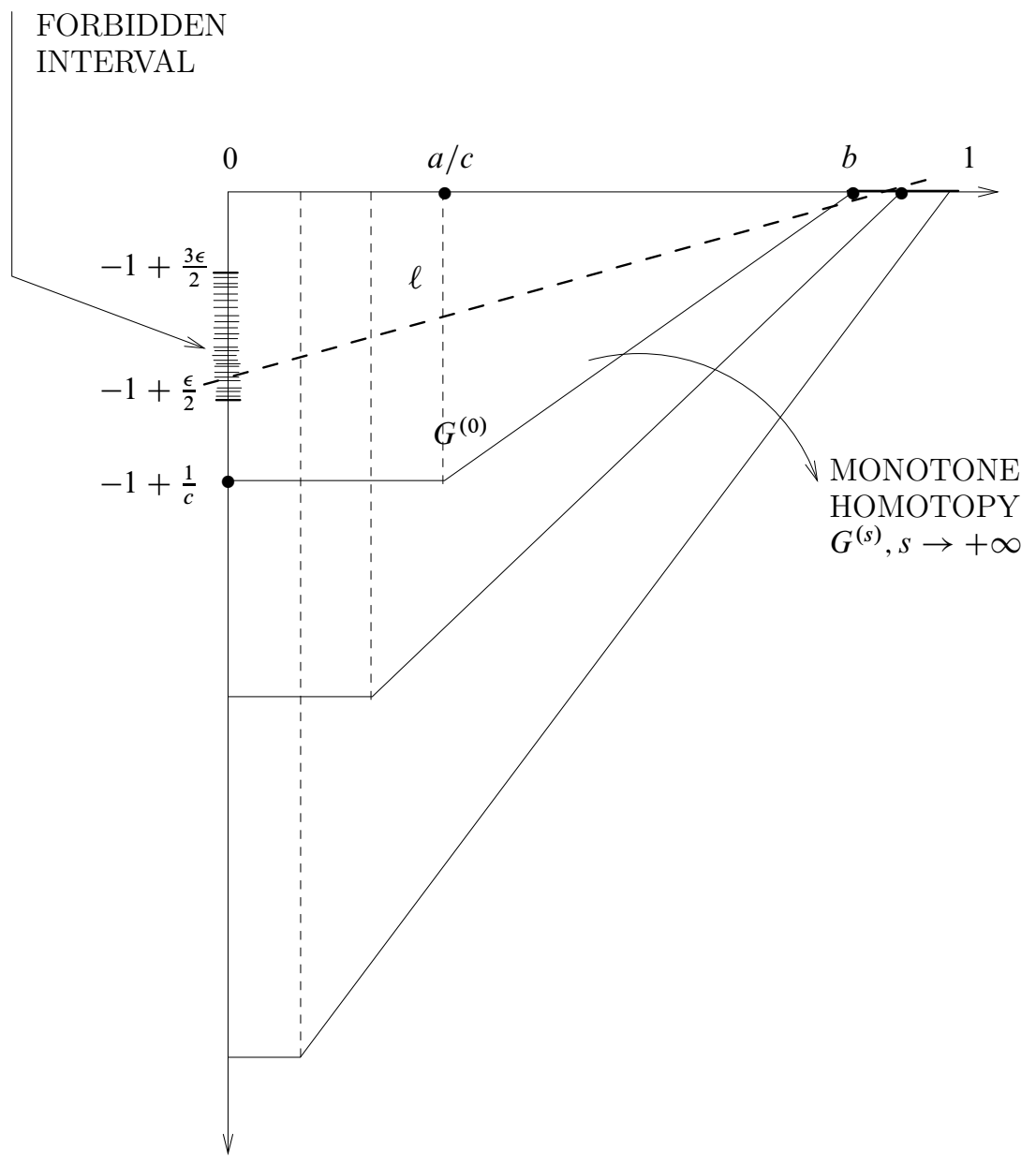

Figure 4

We claim that for all $s$ no line $l \in \mathcal{T}_{\mu(s), \nu(s), \kappa(s)}$ whose slope lies in $-\operatorname{spec}(P)$ can pass through the "forbidden interval" $[-1+\epsilon / 2,-1+3 \epsilon / 2]$ of the vertical axis. Indeed, 
any $l \in \mathcal{T}_{\mu(s), \nu(s), \kappa(s)}$ passing through the "forbidden interval" has the slope close to 1. In particular, this slope lies in $(1-\tau ; 1+\tau)$ for $\epsilon$ small enough and $b$ sufficiently close to 1 . The claim follows from condition (73). Hence we completed the proof of formula (72).

\section{The end of the proof}

Denote $\hat{U}=U \times S^{1}$. Recall that the form $d t-\alpha$ is $\widehat{U}$-basic, see Lemma 4.45 above. Consider a smooth approximation of the function $F_{a, b, c}$ (see Step I) given by $K_{a, b, c}=\overline{H_{a / c, b,-1+1 / c}+1}$ (here the bar stands for the transform described in Step I). Using formulas (66) and (72) we get that

$$
\begin{aligned}
\lim _{\lambda \in \mathcal{F}(\widehat{U}, d t-\alpha)} \mathrm{CH}^{(0, \epsilon)}(\lambda) & =\lim _{\substack{c \rightarrow+\infty \\
a, b \rightarrow 1}} \mathrm{CH}^{(0, \epsilon)}\left(\lambda_{K_{a, b, c}}\right) \\
& =\lim _{\substack{c \rightarrow+\infty \\
a, b \rightarrow 1}} \mathrm{FH}^{(0 ; \epsilon)}\left(H_{a / c, b,-1+1 / c}+1\right) \\
& =\lim _{\substack{c \rightarrow+\infty \\
a, b \rightarrow 1}} \mathrm{FH}^{(-1,-1+\epsilon)}\left(H_{a / c, b,-1+1 / c}\right)=\mathrm{SH}^{(-\infty,-1+\epsilon)}(U),
\end{aligned}
$$

where the limits are understood as direct limits. Finally, we pass to the inverse limit and see that

$$
\begin{aligned}
& \mathrm{CH}(\widehat{U})=\lim _{\varepsilon \rightarrow 0} \lim _{\lambda \in \mathcal{F}(\widehat{U}, d t-\alpha)} \mathrm{CH}^{(0, \epsilon)}(\lambda)= \\
& \lim _{\varepsilon \rightarrow 0} \mathrm{SH}^{(-\infty,-1+\epsilon)}(U)=\mathrm{SH}^{(-\infty,-1)}(U) .
\end{aligned}
$$

In the last equality we use that

$$
\mathrm{SH}^{(-\infty,-1+\epsilon)}(U)=\mathrm{SH}^{(-\infty,-1)}(U)
$$

since $-1 \notin \operatorname{spec}(P)$. This completes the proof.

Proposition 5.6 The isomorphism $\Psi_{U}$ : $\mathrm{CH}\left(U \times S^{1}\right)=\mathrm{SH}^{(-\infty,-1)}(U)$ constructed in Theorem 5.3 is functorial in the following sense. Consider two starshaped domains $U_{1} \subset$ $U_{2} \subset M$ with smooth non-resonant boundaries. Let $I: \mathrm{CH}\left(U_{1} \times S^{1}\right) \rightarrow \mathrm{CH}\left(U_{2} \times S^{1}\right)$ and $l: \mathrm{SH}^{(-\infty ;-1)}\left(U_{1}\right) \rightarrow \mathrm{SH}^{(-\infty,-1)}\left(U_{2}\right)$ be the inclusion homomorphisms in contact and symplectic homologies. Then

$$
\Psi_{U_{2}} \circ I=\imath \circ \Psi_{U_{1}} .
$$

Proof Let us re-examine the isomorphism between $\mathrm{CH}\left(\lambda_{H}\right)$ and $\mathrm{FH}(\bar{H})$ given by formula (66) in Step I of the proof of Theorem 5.3. Consider the following manifolds: 
- the symplectization $S V=V \times \mathbb{R}_{+}$of $(V, \xi)$ equipped with the Liouville form $\Pi=s(d t-\alpha)$;

- the manifold $V \times \mathbb{R}$ endowed with the 1 -form $\Pi^{\prime}=s d t-\alpha$.

Define an embedding $A: S V \rightarrow V \times \mathbb{R}$ by the formula

$$
F(z, t, s)=\left(L^{\log s}(z), t, s\right), \text { where }(z, t) \in V=M \times S^{1}, s \in \mathbb{R}_{+} .
$$

Note that $A^{*} \Pi^{\prime}=\Pi$. Furthermore, on the subset

$$
P \times \mathbb{R}_{+} \times S^{1} \times \mathbb{R}_{+} \subset S V
$$

the map $A$ takes the form

$$
(x, u, t, s) \mapsto(x, s u, t, s) .
$$

Therefore, given a Hamiltonian $H \in \mathcal{H}$, the graph of the contact form $\lambda_{H}:=\frac{1}{H}(d t-\alpha)$, viewed as a section of the symplectization $S V \rightarrow V$, is mapped by $A$ to the hypersurface $\{s=\bar{H}(u)\} \subset V \times \mathbb{R}$. Indeed, this is equivalent to the identity

$$
\bar{H}\left(\frac{u}{H(u)}\right)=\frac{1}{H(u)},
$$

which is dual to (67).

Given two Hamiltonians $H_{1}>H_{2}$ from $\mathcal{H}$, we observe that the domain $Y \subset S V$ between the graphs of the forms $\lambda_{H_{2}}$ and $\lambda_{H_{1}}$ is mapped by $A$ to the domain $Y^{\prime} \subset V \times \mathbb{R}$ between the graphs of Hamiltonians $\bar{H}_{2}$ and $\bar{H}_{1}$. But the domains $Y$ and $Y^{\prime}$ correspond to directed cobordisms which are used in Sections 4.13 and 4.12 in order to define the monotonicity maps in contact and Floer homology theories. Hence, the isomorphism between $\mathrm{CH}\left(\lambda_{H}\right)$ and $\mathrm{FH}(\bar{H})$ is functorial with respect to the monotonicity morphisms. It is straightforward to check that this functorial correspondence survives all the limits in the construction of $\Psi_{U}$.

\subsection{Ellipsoids and balls}

Note All symplectic and contact homology in this section are $\mathbb{Z}$-graded (see Remark 4.10 above).

Proof of Theorem 1.28 Here we calculate the contact homology of the domain $\widehat{E}(N, R)=E(N, R) \times S^{1} \subset \mathbb{C}^{n} \times S^{1}$, where $E(N, R)$ is the ellipsoid

$$
E(N, R):=\left\{\pi\left|z_{1}\right|^{2}+\frac{\pi}{N} \sum_{i=2}^{n}\left|z_{i}\right|^{2}<R\right\}, N \in \mathbb{N}, \frac{1}{R} \notin \mathbb{N} .
$$


Note that $P:=\partial E(N, R)$ is a convex hypersurface in $\mathbb{C}^{n}$. The action spectrum of $P$ equals $-R \mathbb{N}$, hence the condition $\frac{1}{R} \notin \mathbb{N}$ is exactly the non-resonant condition. Therefore one can apply Theorem 5.3. Together with an obvious rescaling it yields

$$
\mathrm{CH}_{*}(\widehat{E}(N, R))=\mathrm{SH}_{*}^{(-\infty,-1)}(E(N, R))=\mathrm{SH}_{*}^{\left(-\infty,-\frac{1}{R}\right)}(E(N, 1)) .
$$

Next we calculate symplectic homology $\mathrm{SH}_{*}^{\left(-\infty,-\frac{1}{R}\right)}(E(N, 1))$ (see [23] for a calculation of another version of symplectic homology for ellipsoids). Define a piecewise linear function

$$
F_{a, c}:[0 ;+\infty) \rightarrow \mathbb{R}, a \in(0,1), c<0
$$

as follows: $F_{a, c} \equiv 0$ on $[a,+\infty), F_{a, c}(0)=c$ and $F_{a, c}$ is linear on $[0, a]$. We consider $F_{a, c}(u)$ as a Hamiltonian on $\mathbb{C}^{n}$ with

$$
u=\pi\left|z_{1}\right|^{2}+\frac{\pi}{N} \sum_{i=2}^{n}\left|z_{i}\right|^{2} .
$$

In order to deal with Floer homology of $F_{a, c}$ we approximate it by a smooth function, exactly as in Step II of the proof of Theorem 5.3 above. We leave the details of the approximation argument to the reader. In what follows $\mathcal{T}_{a, c}$ stands for the set of generalized tangent lines to graph $F_{a, c}$ which consists of the following points $\left(l,\left(u, F_{a, c}(u)\right) \in P T \mathbb{R}^{2}:\right.$

- $l$ is the usual tangent line when $u \neq a$;

- $l$ lies above $\operatorname{graph}\left(F_{a, c}\right)$ when $u=a$.

We claim that

$$
\mathrm{SH}_{*}^{\left(-\infty,-\frac{1}{R}\right)}(E(N, 1))=\mathrm{FH}_{*}^{\left(-\infty,-\frac{1}{R}\right)}\left(F_{a,-\frac{1}{R}-\delta}\right)
$$

provided $a$ is sufficiently close to 1 and $\delta$ is small enough. Indeed, consider a monotone homotopy of $F_{a,-\frac{1}{R}-\delta}$ through a dominating family of functions $F_{a(s), c(s)}, s \geq 0$ (see Figure 5) and note that no line from $\mathcal{T}_{a(s), c(s)}$ which passes through a $\frac{\delta}{2}$-neighborhood of $-1 / R$ on the vertical axis has an integral slope. The claim follows from Lemma 4.39 .

Finally, note that the only 1 -periodic orbit of $F_{a,-\frac{1}{R}-\delta}$ with action $\leq-\frac{1}{R}$ corresponds to the fixed point at $u=0$. In the neighborhood of this point,

$$
F_{a,-\frac{1}{R}-\delta}(z)=\mathrm{const}+\pi\left[\frac{1}{R}\right]\left|z_{1}\right|^{2}+\pi\left[\frac{1}{N R}\right] \sum_{i=2}^{n}\left|z_{i}\right|^{2}+\pi H(z),
$$




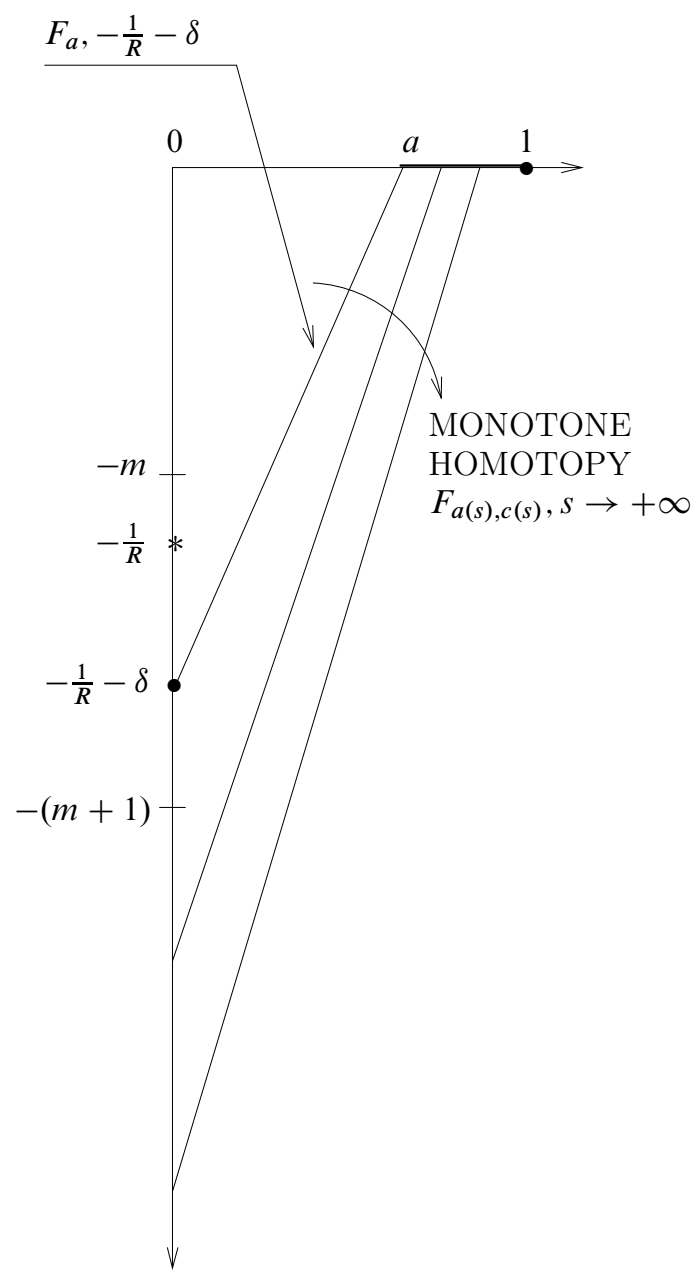

Figure 5

where $H(z)=\sum_{i=1}^{n} c_{i}\left|z_{i}\right|^{2}$ with $c_{i} \in(0,1)$. Therefore, according to our convention (see Section 4.4.1), the Conley-Zehnder index of our fixed point equals

$$
k(N, R)=-2\left[\frac{1}{R}\right]-2(n-1)\left[\frac{1}{N R}\right]
$$

We conclude that $\mathrm{SH}_{i}^{(-\infty,-1 / R)}(E(N, 1))$ equals $\mathbb{Z}_{2}$ for $i=k(N, R)$ and vanishes otherwise. The theorem follows now from equation (76). 
Proof of Theorem 1.29 Assume that

$$
\frac{1}{k}<R_{1}<R_{2}<\frac{1}{k-1}
$$

for some $k \in \mathbb{N}$. Using the functoriality equation (74) we see that it suffices to show that the inclusion $B^{2 n}\left(R_{1}\right) \hookrightarrow B^{2 n}\left(R_{2}\right)$ induces an isomorphism of symplectic homologies

$$
\mathrm{SH}^{(-\infty ;-1)}\left(B^{2 n}\left(R_{1}\right)\right) \rightarrow \mathrm{SH}^{(-\infty ;-1)}\left(B^{2 n}\left(R_{2}\right)\right) .
$$

Consider the family of balls $B_{s}:=B^{2 n}(s), s \in\left[R_{1}, R_{2}\right]$. The desired statement follows from the fact that $-1 \notin \operatorname{spec}\left(B_{S}\right)=-s \mathbb{N}$ for all $s \in\left[R_{1}, R_{2}\right]$ in view of our assumptions on $R_{1}$ and $R_{2}$.

\subsection{Cotangent bundles and free loop spaces}

Note All symplectic and contact homology in this section are $\mathbb{Z}$-graded (see Remark 4.11 above). All singular (co)homology are with $\mathbb{Z}_{2}-$ coefficients.

Let $X$ be a closed manifold, and let $T^{*} X$ be the cotangent bundle of $X$ with the standard symplectic form $\omega=d \alpha$ where $\alpha=p d q$. Consider the contact manifold $\left(V=T^{*} X \times S^{1}, \xi=\operatorname{Ker}(\alpha-d t)\right)$. Choose a Riemannian metric on $X$ and set $U_{r}=$ $\{|p|<r\}$. Thus $U_{r}$ is the ball bundle associated with $T^{*} X$. Denote $\hat{U}_{r}=U_{r} \times S^{1} \subset V$. Write $\Lambda$ for the length spectrum of the metric. Note that the hypersurface $\partial \widehat{U}_{r}$ is non resonant if and only if $r^{-1} \notin \Lambda$. For the sake of brevity, will shall call such values of $r$ non-resonant.

Let us denote by $\mathcal{L} X$ the free loop space of $X$, by $S: \mathcal{L} X \rightarrow \mathbb{R}$ the action functional

$$
S(\gamma)=\frac{1}{2} \int_{0}^{1}\left\|\frac{d \gamma}{d t}\right\|^{2} d t,
$$

and by $\mathcal{L}^{a} X$ the set $\{S \leq a\} \subset \mathcal{L} X$. We will use below the following theorem due to $\mathrm{J}$. Weber [47] which is based on earlier works $[45 ; 1 ; 43]$.

Theorem 5.7 For each non-resonant $r>0$ there exists an isomorphism

$$
L_{r}: \mathrm{SH}^{(-\infty,-1)}\left(U_{r}\right) \rightarrow H^{*}\left(\mathcal{L}^{\frac{1}{2 r^{2}}} X\right)
$$


Furthermore, if $0<r<r^{\prime}$ are non-resonant, the following diagram commutes:

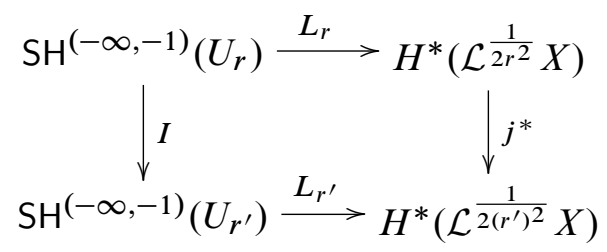

where $I$ and $j^{*}$ are respective inclusion homomorphisms.

Theorem 5.3 then implies:

Corollary 5.8 For each non-resonant $r>0$ there exists an isomorphism

$$
\widehat{L}_{r}: \mathrm{CH}\left(\widehat{U}_{r}\right) \rightarrow H^{*}\left(\mathcal{L}^{\frac{1}{2 r^{2}}} X\right)
$$

such that if $r<r^{\prime}$ the following diagram commutes

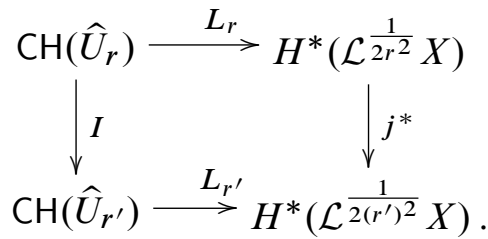

\section{Proof of Theorem 1.18}

Observe that every finite covering $X \rightarrow X^{\prime}$ lifts to a finite covering $\mathbb{P}_{+} T^{*} X \rightarrow$ $\mathbb{P}_{+} T^{*} X^{\prime}$ which is a local contactomorphism. Thus a positive contractible loop in Cont $_{0}\left(\mathbb{P}_{+} T^{*} X^{\prime}\right)$ lifts to a positive contractible loop in Cont ${ }_{0}\left(\mathbb{P}_{+} T^{*} X\right)$. Therefore if $\mathbb{P}_{+} T^{*} X^{\prime}$ is non-orderable then $\mathbb{P}_{+} T^{*} X$ is non-orderable as well. Hence, the case when $\pi_{1}(X)$ is finite can be reduced to the case when $X$ is simply connected.

Consider natural morphisms

$$
\begin{gathered}
\tau_{a}: H^{*}(\mathcal{L} X) \rightarrow H^{*}\left(\mathcal{L}^{a} X\right), \\
\tau_{E, a}: H^{*}\left(\mathcal{L}^{E} X\right) \rightarrow H^{*}\left(\mathcal{L}^{a} X\right), \quad E>a,
\end{gathered}
$$

and denote by $\beta(a)$ and $\beta(E, a)$ the dimensions of their images, respectively. Note that all these functions are non-decreasing in $a$ and non-increasing in $E$.

Lemma 5.9 Assume that $X$ is simply connected, or that $\pi_{1}(X)$ has infinite number of conjugacy classes. Then

(i) $\beta(a) \underset{a \rightarrow \infty}{\rightarrow} \infty$; 
(ii) For every $a>0$ there exists $E(a)$ such that $\beta(E, a)=\beta(a)$ for all $E \geq E(a)$.

The proof is presented at the end of this section.

We continue the proof of Theorem 1.18. According to our covering argument we can assume that $X$ is either simply connected, or $\pi_{1}(X)$ has infinitely many conjugacy classes.

In view of Lemma 5.9(i) one can choose an arbitrarily small $r$ such that $\beta(a)$ jumps when crossing $b=\frac{1}{2 r^{2}}$, that is for any sufficiently small $\delta_{1}, \delta_{2}>0$ we have

$$
\beta\left(b-\delta_{1}\right)<\beta\left(b+\delta_{2}\right) .
$$

We can assume that $b-\delta_{1}$ and $b+\delta_{2}$ are non-resonant. In what follows we fix $\varepsilon>0, r, \delta_{1}$ and $\delta_{2}$ assuming that they are as small as needed. Put $E=1 /\left(2 \varepsilon^{2}\right)$, $r^{-}=1 / \sqrt{2\left(b+\delta_{2}\right)}$ and $r^{+}=1 / \sqrt{2\left(b-\delta_{1}\right)}$.

According to Theorem 1.19, a contractible positive loop of contactomorphisms on $\mathbb{P}_{+} T^{*} X$ allows us to construct a contact squeezing of the domain $\hat{U}_{r}+$ into $\hat{U}_{r^{-}}$. Moreover, this squeezing can be done in the class of fiberwise starshaped domains, and hence it can be performed via a contact isotopy which fixes pointwise a small neighborhood $\hat{U}_{\varepsilon}$ of the zero section. In other words, there exists a contact isotopy $\Phi_{t}: V \rightarrow V, t \in[0,1]$, such that $\Phi_{0}=\mathbf{1}, \Phi_{t} \equiv \mathbf{1}$ on $\widehat{U}_{\varepsilon}$ for all $t$ and

$$
\Phi_{1}\left(\operatorname{Closure}\left(\widehat{U}_{r^{+}}\right)\right) \subset \widehat{U}_{r^{-}} \text {. }
$$

Note that the inclusion homomorphism

$$
I: \mathrm{CH}\left(\widehat{U}_{\varepsilon}\right) \rightarrow \mathrm{CH}\left(\widehat{U}_{r^{-}}\right)
$$

splits as a composition

$$
\mathrm{CH}\left(\widehat{U}_{\varepsilon}\right) \rightarrow \mathrm{CH}\left(\Phi_{1}\left(\widehat{U}_{r+}\right)\right) \rightarrow \mathrm{CH}\left(\widehat{U}_{r^{-}}\right),
$$

and hence

$$
\operatorname{dim} \operatorname{Image}\left(\mathrm{CH}\left(\widehat{U}_{\varepsilon}\right) \rightarrow \mathrm{CH}\left(\Phi_{1}\left(\widehat{U}_{r^{+}}\right)\right)\right) \geq \operatorname{dim} \operatorname{Image}\left(\mathrm{CH}\left(\widehat{U}_{\varepsilon}\right) \rightarrow \mathrm{CH}\left(\widehat{U}_{r^{-}}\right)\right) .
$$

But

$$
\text { Image }\left(\mathrm{CH}\left(\widehat{U}_{\varepsilon}\right) \rightarrow \mathrm{CH}\left(\Phi_{1}\left(\widehat{U}_{r^{+}}\right)\right)\right) \simeq \operatorname{Image}\left(\mathrm{CH}\left(\widehat{U}_{\varepsilon}\right) \rightarrow \mathrm{CH}\left(\widehat{U}_{r^{+}}\right)\right) .
$$

According to the commutative diagram in Corollary 5.8 this yields that

$$
\beta\left(E, b-\delta_{1}\right) \geq \beta\left(E, b+\delta_{2}\right) .
$$


Note that $E$ can be taken arbitrarily large, and hence Lemma 5.9(ii) yields

$$
\beta\left(b-\delta_{1}\right) \geq \beta\left(b+\delta_{2}\right)
$$

in contradiction with inequality (80). This contradiction proves the theorem.

Remark 5.10 Note that if $\pi_{1}(X)$ has infinitely many conjugacy classes it is sufficient to use only 0 -dimensional cohomology. The jump points of the function $\beta(a)$ have in this case a transparent geometric meaning: they correspond to $\ell(\alpha)^{2} / 2$ where $\alpha$ runs over all non-trivial free homotopy classes of loops on $X$ (that is non-trivial conjugacy classes in $\left.\pi_{1}(X)\right)$ and $\ell(\alpha)$ is the minimal length of closed geodesic in $\alpha$.

Proof of Lemma 5.9 The case when $\pi_{1}(X)$ has infinitely many conjugacy classes is obvious already on the level of 0 -dimensional cohomology. To deal with the simply connected case let us recall some facts from the theory of free loop spaces:

(I) The space $H^{*}(\mathcal{L} X)$ has infinite dimension. Let us emphasize that we are dealing here with $\mathbb{Z}_{2}$ coefficients. This is proved in [20].

(II) $H^{*}(\mathcal{L} X)$ is the inverse limit of $H^{*}\left(\mathcal{L}^{E} X\right)$ as $E \rightarrow \infty$ (see eg [47]).

First we prove statement (i) of the lemma. It is more convenient to work here with homology instead of cohomology. Any homology class $\alpha \in H_{*}(\mathcal{L} X)$ can be represented by a cycle contained in $\mathcal{L}^{a} X$ for some finite $a$. Thus the number

$$
A(\alpha)=\inf \left\{a \mid \alpha \in \operatorname{Image}\left(\tau_{a}^{*}\right)\right\},
$$

where $\tau_{a}^{*}$ stands for the natural morphism

$$
H_{*}\left(\mathcal{L}^{a} X\right) \rightarrow H_{*}(\mathcal{L} X),
$$

is well defined for every $\alpha \in H_{*}(\mathcal{L} X)$. Since $\tau_{a}^{*}$ factors through $H_{*}\left(\mathcal{L}^{b} X\right)$ for all $b>a$ we have that $\alpha \in \operatorname{Image}\left(\tau_{b}^{*}\right)$ provided $b>A(\alpha)$. Take any $N>0$. Using (I), choose $N$ linearly independent cycles $\alpha_{1}, \ldots, \alpha_{N} \in H_{*}(\mathcal{L} X)$. It follows that

$$
\beta(a)=\operatorname{dim} H_{*}\left(\mathcal{L}^{a} X\right) \geq N
$$

provided $a>\max _{i} A\left(\alpha_{i}\right)$. Thus

$$
\beta(a) \underset{a \rightarrow \infty}{\rightarrow} \infty
$$

as required.

Let us prove statement (ii) of the lemma. Put

$$
\beta(\infty, a)=\lim _{E \rightarrow \infty} \beta(E, a) .
$$


The limit exists since $\beta(E, a)$ is a non-increasing function of $E$. Observe that $\beta(E, a) \geq \beta(a)$ for all sufficiently large $E$, and therefore

$$
\beta(\infty, a) \geq \beta(a) .
$$

For every $c>0, H^{*}\left(\mathcal{L}^{c} X\right)$ is a finite-dimensional space over $\mathbb{Z}_{2}$, since $\mathcal{L}^{c} X$ has homotopy type of a finite $C W$-complex. Thus the family of decreasing subspaces Image $\left(\tau_{E, c}\right)$ stabilizes to a subspace $F_{c} \subset H^{*}\left(\mathcal{L}^{c} X\right)$ when $E \rightarrow \infty .^{5}$ Clearly, $\operatorname{dim} F_{a}=\beta(\infty, a)$. Note that $\tau_{p, q}$ sends $F_{p}$ onto $F_{q}$ for $p>q$. Consider the following commutative diagram:

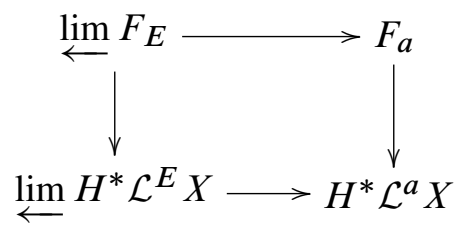

Here the vertical arrows are natural inclusions and the horizontal arrows are natural projections. The upper horizontal arrow is surjective, while the inverse limit in the south-west corner equals $H^{*}(\mathcal{L} X)$ by (II). Thus $\beta(a) \geq \beta(\infty, a)$, and so in view of inequality (82) we have $\beta(a)=\beta(\infty, a)$. Since $\beta(E, a)$ is an integer, we conclude that the function $\beta(E, a)$ equals $\beta(a)$ for a sufficiently large $E$. This completes the proof of (ii).

\section{Discussion and further directions}

Here we touch miscellaneous topics related to geometry of contact domains and transformations. We discuss (non)-squeezing of contact domains from the viewpoint of quantum mechanics. Then we speculate on links between orderability and fillability of contact manifolds. Finally, we introduce a canonical semigroup associated to a contact manifold. This semigroup carries footprints of a partial order even when the manifold is non-orderable.

\subsection{Contact non-squeezing at the quantum scale}

We start with a brief (and standard) discussion of quasi-classical approximation in quantum mechanics. Consider the Cauchy problem for the Schrödinger equation

$$
i \hbar \frac{\partial \psi}{\partial t}=-\frac{1}{2} \hbar^{2} \Delta \psi+W(q) \psi
$$

\footnotetext{
${ }^{5}$ This is called the Mittag-Leffler condition in homological algebra.
} 
which describes the quantum motion of a mass 1 particle on $\mathbb{R}^{n}$ in the presence of a potential force $F=-\frac{\partial W}{\partial q}$. Here $\hbar$ is the Planck constant. Assume that the initial complex-valued wave function is given in the form

$$
\psi_{0}(q)=\rho_{0}^{\frac{1}{2}}(q) e^{i F_{0}(q) / \hbar} .
$$

Here $\rho_{0}$ is the probability density of the particle position in $\mathbb{R}^{n}$ and $F_{0}: \mathbb{R}^{n} \rightarrow S_{\hbar}^{1}$ is the phase which takes values in the circle $S_{\hbar}^{1}=\mathbb{R} /(2 \pi \hbar \mathbb{Z})$.

Quasi-classical approximation gives the following recipe for an approximate solution of the Cauchy problem which involves contact geometry and dynamics: Consider the contact manifold $\left(V_{\hbar}=\mathbb{R}^{2 n} \times S_{\hbar}^{1}, \zeta\right)$ where $\zeta=\operatorname{Ker}(d u-p d q)$. Consider the lift $f_{t}: V_{\hbar} \rightarrow V_{\hbar}$ of the Hamiltonian flow on $\mathbb{R}^{2 n}$ describing the classical motion of our particle. The flow $f_{t}$, which is given by the system

$$
\left\{\begin{array}{l}
\dot{q}=p \\
\dot{p}=-\frac{\partial W}{\partial q} \\
\dot{u}=\frac{1}{2} p^{2}-W(q)
\end{array}\right.
$$

preserves the contact structure $\zeta$.

Given a function $K: \mathbb{R}^{n} \rightarrow S_{\hbar}^{1}$ and a probability density $\rho(q) d q$ on $\mathbb{R}^{n}$, consider a Legendrian submanifold

$$
L(K)=\left\{u=K(q, 0), p=\frac{\partial K(q, 0)}{\partial q}\right\} \subset V
$$

equipped with the density $\sigma(\rho):=\tau^{*}(\rho(q) d q)$ where $\tau: V \rightarrow \mathbb{R}^{n}$ stands for the natural projection. For $t$ small enough, the image $f_{t}\left(L\left(F_{0}\right)\right)$ can be written as $L\left(F_{t}\right)$ and the density $f_{t *} \sigma\left(\rho_{0}\right)$ can be written as $\sigma\left(\rho_{t}\right)$. The main outcome of this construction is as follows. The wave function

$$
\psi_{t}(q)=\rho_{t}^{\frac{1}{2}}(q) e^{i F_{t}(q) / \hbar}
$$

is an approximate solution of the Schrödinger equation (83) in the following sense: it satisfies the Schrödinger equation up to higher order terms in $\hbar$.

The above discussion suggests that it is worth studying contact geometry and dynamics on the manifold $\left(V_{\hbar}, \zeta\right)$ keeping track of the value of the Planck constant $\hbar$. In what follows we reexamine our (non)-squeezing results from this viewpoint. Put $h=2 \pi \hbar$ and for a domain $U \subset \mathbb{R}^{2 n}$ define its prequantization as $\widehat{U}_{\hbar}=U \times S_{\hbar}^{1}$. Next note that the map

$$
\Phi: V \rightarrow V_{h}, \quad(p, q, t) \rightarrow\left(\sqrt{h} p, \sqrt{h} q, h\left(t+\frac{1}{2} p \cdot q\right)\right)
$$


establishes a contactomorphism between $(V, \xi)$ and $\left(V_{\hbar}, \zeta\right)$. Furthermore, given a domain $U \subset \mathbb{R}^{2 n}$,

$$
\Phi(\hat{U})=(\hat{\sqrt{h}} \cdot U)_{\hbar}
$$

Therefore, after a rescaling, our squeezing and non-squeezing results presented in Sections 1.1 and 1.2 extend to the contact manifold $\left(V_{\hbar}, \zeta\right)$. In particular, prequantization of balls $B^{2 n}(R)$ of sub-quantum size $R<h$ in the classical phase space gives rise to squeezable domains $\widehat{B}_{\hbar}^{2 n}(R)$ in $V_{\hbar}$. One can say that on the sub-quantum scale, symplectic rigidity is lost after prequantization. On the other hand, rigidity of balls in the classical phase space persists after prequantization provided the size of the ball is an integer multiple of $h: \widehat{B}^{2 n}(m h)$ cannot be squeezed into itself. It would be interesting to explore whether this geometric phenomenon has some physical meaning.

Note that the balls $B^{2 n}(R)$ are energy levels of the classical harmonic oscillator, while the sequence $\{m h\}$ is, in accordance to Planck's hypothesis, the energy spectrum of the quantized harmonic oscillator. (Planck's hypothesis, which appeared at the early stage of development of quantum mechanics, turned out to be non-precise and was later on corrected in a more advanced model. Still, it gives a satisfactory approximation to the energy spectrum.) It is unclear whether appearance of the sequence $\{m h\}$ in the context of (non)-squeezing is occasional or not. A possibility, which is still open, is that $\widehat{B}_{\hbar}^{2 n}(R)$ cannot be squeezed into itself for any $R>h$.

In $[12 ; 13]$ de Gosson revised the classical notion of quantum cell in the phase space $\mathbb{R}^{2 n}$ in terms of symplectic capacities. The following feature of de Gosson's definition is of interest in our context: for a quantum cell $U$, both $\underline{c}(U)$ and $\bar{c}(U)$ are of order $\hbar$. Interestingly enough, the same scale appears in our (non)-squeezing problem. To be more precise, let us introduce the following notion which is natural when one studies transition from squeezing to non-squeezing in families of domains. Fix a constant $D>0$ and consider the class $\Xi_{D}$ of domains $U \subset \mathbb{R}^{2 n}$ with $\bar{c}(U) \leq D \cdot \underline{c}(U)$. For instance, all convex domains lie in $\Xi_{D}$ for $D=4 n^{2}$ (see [46],[11]). We say that a domain $U \in \Xi_{D}$ is critical if $\hat{U}_{\hbar}$ is negligible (that is $\hat{U}_{\hbar}$ can be contactly squeezed into $\widehat{B}_{\hbar}^{2 n}(r)$ for all $\left.r>0\right)$, while $(2 \cdot \hat{\cdot})_{\hbar}$ is not negligible. Theorem 1.7 yields that $\underline{c}(U) \sim \hbar$ for every critical domain $U$.

\subsection{Fillability and orderability}

Conjecture 6.1 If a contact manifold $\left(P^{2 n-1}, \eta\right)$ admits a Weinstein filling $W^{2 n}$ with $H_{n}(W) \neq 0$ then it is orderable.

This conjecture is supported by spaces of co-oriented contact manifolds, see Theorem 1.18 above. If this conjecture were true then together with Corollary 1.17 it would 
leave the orderability problem for Weinstein fillable contact manifolds open essentially only in the 1-subcritical case, eg, for $S^{2} \times S^{1}$ with its tight contact structure.

Note that the real projective space $P:=\mathbb{R} P^{2 n-1}$ with the standard contact structure, which is orderable by Givental's theorem (see [19]), is not Weinstein fillable for any $n>2$ (though it is symplectically fillable). Indeed, suppose that $W$ is such a filling. Then

$$
H^{2}\left(W, P ; \mathbb{Z}_{2}\right)=H_{2 n-2}\left(W ; \mathbb{Z}_{2}\right)=0
$$

for $2 n-2>n$, or $n>2$. Looking at the cohomology long exact sequence

$$
H^{1}\left(W, \mathbb{Z}_{2}\right) \stackrel{r}{\longrightarrow} H^{1}\left(P, \mathbb{Z}_{2}\right) \longrightarrow H^{2}\left(W, P ; \mathbb{Z}_{2}\right)=0
$$

we see that the map $r$ is onto and hence there is a class $a \in H^{1}\left(W ; \mathbb{Z}_{2}\right)$ such that $b=r(a)$ is the generator of $H^{1}\left(P ; \mathbb{Z}_{2}\right)$. Hence, we have $b^{2 n-1} \neq 0$ which contradicts to the fact that $a^{2 n-1} \in H^{2 n-1}\left(W ; \mathbb{Z}_{2}\right)=0$. It would be interesting to study orderability of more general contact manifolds which are not Weinstein fillable. It seems likely that the methods of the current paper extend to a more general case of symplectically fillable contact manifolds.

The case $n=2$ corresponds to the real projective 3 -space $\mathbb{R} P^{3}$ which is contactomorphic to the space of co-oriented contact elements of $S^{2}$. It is orderable in view of Theorem 1.18.

\subsection{Contact diffeomorphisms and semigroups}

6.3.1 Semigroups and orderability Let $(P, \eta)$ be a closed contact manifold. Denote by $G$ the group $\operatorname{Cont}_{0}(P, \eta)$ and by $\widetilde{G}$ its universal cover. We will often identify (paths of) contactomorphisms of $P$ with the corresponding (paths of) $\mathbb{R}_{+}$-equivariant symplectomorphisms of the symplectization $S P$.

Consider the set of positive paths

$$
f=\left\{f_{t}\right\}, t \in[0,1], f_{0}=\mathbf{1},
$$

on $G$ (that is the paths generated by positive contact Hamiltonians). We say that two such paths $f^{\prime}$ and $f^{\prime \prime}$ are equivalent if $f_{1}^{\prime}=f_{1}^{\prime \prime}$ and the paths are homotopic through positive paths with fixed end points. Denote by $\vec{G}=\vec{G}(P, \eta)$ the set of equivalence classes. ${ }^{6}$ The operation

$$
\left[\left\{f_{t}\right\}\right] \circ\left[\left\{g_{t}\right\}\right]=\left[\left\{f_{t} g_{t}\right\}\right],
$$

\footnotetext{
${ }^{6}$ When necessary, we can assume without loss of generality that our paths are generated by 1 -periodic in time contact Hamiltonians, see Lemma 3.1.A in [19].
} 
where $\left\{f_{t}\right\}$ and $\left\{g_{t}\right\}$ are positive paths, defines a semigroup structure on $\vec{G}$. Note that $\vec{G}$ comes along with the natural projections $\tau: \vec{G} \rightarrow \widetilde{G}$ and $\pi: \vec{G} \rightarrow G$ which are morphisms of semigroups.

The (non)-orderability of $(P, \eta)$ can be recognized in terms of $\vec{G}$ and $\tau$ as follows. Denote by $\tilde{\mathbf{1}} \in \widetilde{G}$ the canonical lift of the identity. When $(P, \eta)$ is orderable, there are no positive contractible loops on $G$ and hence $\tilde{\mathbf{1}}$ does not belong to the image of $\tau$. In contrast to this, when $(P, \eta)$ is not orderable the morphism $\tau$ is surjective. Indeed, in this case we have a positive contractible loop $\left\{\phi_{t}\right\}$ of contactomorphisms with $\phi_{0}=\phi_{1}=1$. Thus for every path of contactomorphisms $f=\left\{f_{t}\right\}$ on $G$ starting at the identity, the path $h=\left\{f_{t} \phi_{t}^{N}\right\}$ is positive for $N$ large enough, and hence we get an element $[h] \in \vec{G}$ with $\tau([h])=[f] \in \widetilde{G}$.

6.3.2 Partial order for non-orderable manifolds Assume now that $(P, \eta)$ is nonorderable. In this case

$$
\vec{G}_{0}:=\tau^{-1}(\tilde{\mathbf{1}}) \subset \vec{G}
$$

is a subsemigroup. Its elements represent connected components of the space of positive contractible loops on $G$ based at $\mathbf{1}$.

Proposition 6.2 The center of $\vec{G}(P, \eta)$ contains $\vec{G}_{0}(P, \eta)$.

The proposition immediately follows from the formula:

$$
\left[\left\{f_{t} g_{t}\right\}\right]=\left[\left\{g_{t} g_{1}^{-1} f_{t} g_{1}\right\}\right],
$$

where $\left\{f_{t}\right\}$ and $\left\{g_{t}\right\}, t \in[0,1]$, are arbitrary positive paths with $f_{0}=g_{0}=\mathbf{1}$. To prove this formula, define new paths

$$
f_{t}^{\prime}=\left\{\begin{array}{ll}
1 & \text { for } t \in\left[0, \frac{1}{2}\right] ; \\
f_{2 t-1} & \text { for } t \in\left[\frac{1}{2}, 1\right]
\end{array} \text { and } g_{t}^{\prime}=\left\{\begin{array}{ll}
g_{2 t} & \text { for } t \in\left[0 ; \frac{1}{2}\right] ; \\
g_{1} & \text { for } t \in\left[\frac{1}{2}, 1\right]
\end{array}\right. \text {. }\right.
$$

Note now that

$$
f_{t}^{\prime} g_{t}^{\prime}=g_{t}^{\prime} g_{1}^{-1} f_{t}^{\prime} g_{1}
$$

for all $t$. Furthermore, $\left\{f_{t}\right\}$ and $\left\{f_{t}^{\prime}\right\}$ (respectively, $\left\{g_{t}\right\}$ and $\left\{g_{t}^{\prime}\right\}$ ) are homotopic with fixed end points such that all the intermediate paths except $\left\{f_{t}^{\prime}\right\}$ (respectively, $\left.\left\{g_{t}^{\prime}\right\}\right)$ are smooth and positive. Observe that $\left\{f_{t}^{\prime}\right\}$ and $\left\{g_{t}^{\prime}\right\}$ are piece-wise smooth and non-negative. By an appropriate "smoothing of corners" argument, we correct this and get the desired homotopy between $\left\{f_{t} g_{t}\right\}$ and $\left\{g_{t} g_{1}^{-1} f_{t} g_{1}\right\}$ through smooth positive paths, thus proving formula (84).

An interesting feature of the semigroup $\vec{G}_{0}$ is given by the following proposition. 
Proposition 6.3 For every $\gamma \in \vec{G}$ and $\theta \in \vec{G}_{0}$ there exists $N \in \mathbb{N}$ such that $\theta \gamma^{N}=$ $\gamma^{N}$.

Proof Assume that $\gamma$ is represented by a path $\left\{h_{t}\right\}$ generated by a contact Hamiltonian $H(z, t)>0$ and $\theta$ is represented by a loop $f=\left\{f_{t}\right\}$ generated by the contact Hamiltonian $F(z, t)>0$. Let $f^{(s)}=\left\{f_{s, t}\right\}, s \in[0,1]$, be a homotopy of $f=f^{(0)}$ to the constant loop, and let $F_{S}(z, t)$ be the corresponding contact Hamiltonians. Choose $N$ large enough so that

$$
F_{s}\left(f_{t, s} y, t\right)+N H(y, N t)>0
$$

for all $s, t \in[0,1]$ and $y \in S P$. Consider the homotopy of loops $\left\{f_{t, s} \circ h_{t}^{N}\right\}$ which joins $\left\{f_{t} h_{t}^{N}\right\}$ and $\left\{h_{t}^{N}\right\}$. The intermediate loops are generated by the Hamiltonians

$$
F_{S}(z, t)+N H\left(f_{s, t}^{-1} z, N t\right)
$$

which are positive due to our choice of $N$. Thus $\theta \gamma^{N}=\gamma^{N}$ as required.

Corollary 6.4 For every $\theta \in \vec{G}_{0}$ there exists $N \in \mathbb{N}$ such that $\theta^{N+1}=\theta^{N}$.

The corollary implies that for each $\theta \in \vec{G}_{0}$ the sequence $\left\{\theta^{N}\right\}, N \rightarrow+\infty$, stabilizes to an element which we denote by $\bar{\theta}$.

Corollary 6.5 For any $\gamma, \theta \in \vec{G}_{0}$ we have $\bar{\gamma}=\bar{\theta}$.

Indeed, according to Propositions 6.3, 6.2 and Corollary 6.4 we have

$$
\bar{\gamma}=\bar{\theta} \bar{\gamma}=\bar{\gamma} \bar{\theta}=\bar{\theta}
$$

Corollary 6.6 There exists a unique "stable" element in $\vec{G}_{0}$, denoted by $\theta_{\text {st }}$, which is equal to $\bar{\theta}$ for any $\theta \in \vec{G}_{0}$. The element $\theta_{\text {st }}$ can be characterized by any of the following properties:

- $\theta_{\mathrm{st}}$ is the unique idempotent, $\theta_{\mathrm{st}}^{2}=\theta_{\mathrm{st}}$, in the semigroup $\vec{G}_{0}$;

- $\theta_{s t}$ is the zero element of $\vec{G}_{0}$, that is for any $\gamma \in \vec{G}_{0}$ we have

$$
\gamma \theta_{\mathrm{st}}=\theta_{\text {st }} \gamma=\theta_{\text {st }}
$$

Thus we established that some power of every element in $\vec{G}_{0}$ is equal to the zero element. Such semigroups are called nilsemigroups. 
Example 6.7 Consider the semigroup $\vec{G}_{0}\left(S^{2 n-1}\right)$ associated with the standard contact sphere, where $n \geq 2$. The stable element $\theta_{\text {st }}$ can be explicitly identified in this case. Let $\theta \in \vec{G}_{0}$ be the element represented by the positive contractible loop $\varphi=\left\{\varphi_{t}\right\}$ constructed in Theorem 3.6. We claim that $\theta$ is an idempotent: $\theta^{2}=\theta$, and hence $\theta$ coincides with $\theta_{\text {st }}$. To prove this recall that $\left\{\varphi_{t}\right\}$ is generated by the Hamiltonian $\Phi(z, t)$ with $\Phi(z, t)>\pi|z|^{2}$ (see equation (43) above). On the other hand, we have seen in the proof of Theorem 1.11(ii) that there exists a homotopy $\varphi^{(s)}=\left\{\varphi_{t, s}\right\}$ of $\varphi=\varphi^{(0)}$ to the constant loop $\varphi^{(1)} \equiv \mathbf{1}$ such that the Hamiltonians $\Phi_{s}(z, t)$ generating the loops $\varphi^{(s)}$ satisfy $\Phi_{s}(z, t) \geq-\pi|z|^{2}$. Therefore,

$$
\Phi\left(z, t_{1}\right)+\Phi_{s}\left(z, t_{2}\right)>0
$$

for all $z \in \mathbb{C}^{n} \backslash\{0\}, t_{1}, t_{2} \in S^{1}$ and $s \in[0,1]$.

Let $\theta^{*} \in \vec{G}_{0}$ be the element represented by the loop $\varphi^{*}=\left\{\varphi_{1-t}^{-1}\right\}$. Consider the homotopy of $\varphi^{*} \varphi$ to $\varphi$ given by $\left\{\varphi_{1-t, s}^{-1} \varphi_{t}\right\}$, where $s \in[0,1]$. The Hamiltonians of the intermediate loops are given by

$$
\Phi_{s}\left(\varphi_{1-t, s} z, 1-t\right)+\Phi\left(\varphi_{1-t, s} z, t\right) .
$$

These Hamiltonians are positive in view of (85), and therefore $\theta^{*} \theta=\theta$.

Consider now the homotopy of $\varphi^{*} \varphi$ to $\varphi^{*}$ given by $\left\{\varphi_{1-t}^{-1} \varphi_{t, s}\right\}$, where $s \in[0,1]$. The Hamiltonians of the intermediate loops are given by

$$
\Phi\left(\varphi_{1-t} z, 1-t\right)+\Phi_{s}\left(\varphi_{1-t} z, t\right) .
$$

Again, these Hamiltonians are positive in view of (85), and therefore $\theta^{*} \theta=\theta^{*}$. We conclude that $\theta^{*}=\theta$ and hence

$$
\theta^{2}=\theta^{*} \theta=\theta
$$

and the claim is proved.

Remark 6.8 No complete description of the semigroup $\vec{G}_{0}$ is available even in the simplest case when $P$ is the three-dimensional sphere $S^{3}$ equipped with the standard contact structure. In fact, we do not know a single example when $\vec{G}_{0}$ consists of more than just the zero element $\theta_{\mathrm{st}}$.

The next proposition describes in more detail the structure of the semigroup $\vec{G}$. Set

$$
\Gamma=\left\{\gamma \in \vec{G}: \gamma \theta_{\mathrm{st}}=\gamma\right\} .
$$

Proposition 6.9 $\quad$ (i) $\Gamma$ is a subgroup of $\vec{G}$ with the neutral element $\theta_{\mathrm{st}}$; 
(ii) the restriction of the natural projection $\tau: \vec{G} \rightarrow \widetilde{G}$ to $\Gamma$ is a group isomorphism;

(iii) for every $x \in \vec{G}$ there exists $N \in \mathbb{N}$ such that $x^{N} \in \Gamma$ (and hence $\vec{G}$ is an epigroup in the language of semigroup theory);

(iv) $\Gamma$ is a two-sided ideal in $\vec{G}$, that is for every $x \in \vec{G}$ and $\gamma \in \Gamma$ we have $x \gamma, \gamma x \in \Gamma$.

Proof Clearly, $\Gamma$ is closed under the semigroup multiplication. By the definition of $\Gamma$, the element $\theta_{\text {st }}$ is its neutral element. Recall that the morphism $\tau: \vec{G} \rightarrow \widetilde{G}$ is onto. Thus, given any $x \in \Gamma$, we can choose $y \in \vec{G}$ with $\tau(y)=\tau(x)^{-1}$. This yields $\tau(x y)=\tau(y x)=\tilde{\mathbf{1}}$, and hence $x y, y x \in \vec{G}_{0}$. Put $y^{\prime}=y \theta_{\mathrm{st}}$. Note that $y^{\prime} \in \Gamma$ since $\theta_{s t}^{2}=\theta_{s t}$. Recalling that $\theta_{s t}$ is the zero element of $\vec{G}_{0}$, we get

$$
x y^{\prime}=x y \theta_{\mathrm{st}}=\theta_{\mathrm{st}} \text {, and } y^{\prime} x=y \theta_{s t} x=y x \theta_{s t}=\theta_{s t} .
$$

Thus $y^{\prime}$ is the inverse of $x$ in $\Gamma$, and this proves Property (i). Taking into account that $x \theta_{s t} \in \Gamma$ and $\tau\left(x \theta_{s t}\right)=\tau(x)$ for every $x \in \vec{G}$, we get that $\left.\tau\right|_{\Gamma}$ is onto since $\tau$ is onto. Let us show that the kernel of the homorphism $\left.\tau\right|_{\Gamma}$ is trivial. Indeed, take any $\theta \in\left(\left.\tau\right|_{\Gamma}\right)^{-1}(\widetilde{\mathbf{1}})=\Gamma \cap \vec{G}_{0}$. Then we have $\theta \theta_{\text {st }}=\theta$ by the definition of $\Gamma$ and $\theta \theta_{\text {st }}=\theta_{\text {st }}$ since $\theta_{\text {st }}$ is the zero element of $\vec{G}_{0}$. Hence, $\theta=\theta_{\text {st }}$, and we get Property (ii). Property (iii) follows from Proposition 6.3. Property (iv) is a straightforward consequence of the definitions.

Let us introduce a new relation on the semigroup $\vec{G}$. Given $x, y \in \vec{G}$ we say that $x \succeq y$ whenever either $x=y$ or $x=z y$ for some $z \in \vec{G}$.

Proposition 6.10 The relation $\underset{\succ}{\succeq}$ is

(i) reflexive and transitive;

(ii) trivial on $\Gamma$, ie, $x \succeq y$ and $y \succeq x$ for any $x, y \in \Gamma$;

(iii) satisfies the condition $x \underset{+}{\succeq}$ for any $x \in \Gamma$ and $y \in \vec{G} \backslash \Gamma$;

(iv) defines a genuine partial order on $\vec{G} \backslash \Gamma$.

Proof Property (i) is obvious. Property (ii) follows from the fact that $\Gamma$ is a subgroup of $\vec{G}$. Take any $x \in \Gamma$ and $y \in \vec{G} \backslash \Gamma$. Recall that $y \theta_{s t} \in \Gamma$. Using Properties (i) and (ii) we get

$$
x \underset{+}{\succeq} \theta_{s t} \succeq y,
$$


which proves Property (iii). Let us check now that the relation $\underset{+}{\succeq}$ is anti-symmetric on $\vec{G} \backslash \Gamma$. Suppose that $x \succeq y$ and $y \succeq x$ for some $x, y \in \vec{G} \backslash \Gamma$. Assume on the contrary that $x \neq y$. Then $x=a y$ and $y=b x$ for some $a, b \in \vec{G}$. Thus $x=a b x$, and so $\tau(a b)=\tilde{\mathbf{1}}$, which implies that $\theta:=a b \in \vec{G}_{0}$. Multiplying the equation $x=\theta x$ by successive powers of $\theta$ we have

$$
x=\theta x=\ldots=\theta^{N} x=\theta_{\text {st }} x
$$

provided $N$ is large enough. We get a contradiction with our assumption $x \notin \Gamma$. Thus the remaining possibility is $x=y$, and we proved Property (iv).

Since $\Gamma$ is a two-sided ideal of $\vec{G}$, we can consider the Rees quotient semigroup $\vec{G} / \Gamma$. By definition, this is a new semigroup on the set $\mathcal{R}=(\vec{G} \backslash \Gamma) \cup\{0\}$ in which the product of elements $x, y \in \mathcal{R}$ concides with their product $x y$ in $\vec{G}$ if $x, y, x y \in \vec{G} \backslash \Gamma$, and equals 0 otherwise. Property 6.9(iii) yields that for every element $r$ of $\mathcal{R}$ there exists $N \in \mathbb{N}$ such that $r^{N}=0$. Proposition 6.10 implies that the relation $\succeq$ on $\vec{G} \backslash \Gamma$ defines a genuine partial order on $\mathcal{R}$, denoted in the same way, where we declare that $0 \succeq r$ for every $r \in \mathcal{R}$.

Proposition 6.11 The partial order $\succeq$ on $\mathcal{R}$ is bi-invariant: if $x \underset{+}{\succeq}$ for some $x, y \in \mathcal{R}$ then $x c \succeq y c$ and $c x \underset{+}{\succeq} c y$ for every $c \in \mathcal{R}$.

Before proving the proposition let us make the following general remark. The group $G$ acts on $\vec{G}$ by conjugations, that is the action of an element $g \in G$ is given by

$$
A_{g}\left(\left[\left\{f_{t}\right\}\right]\right):=\left[\left\{g^{-1} f_{t} g\right\}\right] .
$$

This action preserves point-wise the subsemigroup $\vec{G}_{0}$ and leaves invariant the subgroup $\Gamma$. Formula (84) above yields

$$
x y=y \cdot A_{\pi(y)} x=A_{\pi(x)}^{-1} y \cdot x
$$

for all $x, y \in \vec{G}$.

Proof of Proposition 6.11 Assume that $x, y \in \mathcal{R}$ with $x \underset{+}{\succeq}$. Suppose that $x \neq 0$ (the case $x=0$ is trivial). By definition, $x=z y$ for some $z \in \vec{G}$. Given any $c \in \mathcal{R}$ 
we have $x c=z y c$ and hence $x c \succeq y c$. Further, $x=y w$ with $w=A_{\pi(y)} z$ in view of formula (86). Applying this formula again, we get

$$
c x=c y w=A_{\pi(c y)}^{-1} w \cdot c y
$$

and hence $c x \succeq c y$.

6.3.3 Semigroup $\mathcal{R}$ and contact squeezing A priori, the set $\vec{G} \backslash \Gamma$ might be empty even for non-orderable contact manifolds (though we are not aware of such an example). Interestingly enough, in order to prove that it is non-empty for the standard contact sphere (see Proposition 6.13 below), we use the contact non-squeezing theorem. This suggests that non-triviality of the partially ordered semigroup $\mathcal{R}$ is a "hard" aspect of the "soft" non-orderability phenomenon.

Let us begin with a general setting. Recall that whenever $(P, \eta)$ is an ideal contact boundary of a Liouville manifold $(M, \omega, L)$, Lemma 1.21 establishes a correspondence between elements $\gamma \in \vec{G}(P, \eta)$ and fiberwise starshaped domains $U(\gamma) \subset M \times S^{1}$ up to contact isotopy. Generalizing the definition given in Section 1.2, we say that a subset of $M \times S^{1}$ is negligible if it can be contactly squeezed into an arbitrarily small neighborhood of $\operatorname{Core}(M) \times S^{1}$.

Proposition 6.12 For any $\gamma \in \Gamma$ the domain $U(\gamma)$ is negligible.

Proof Take any positive time-independent contact Hamiltonian $E$ on $S P$. The claim can be equivalently expressed by saying that for any $C>0$ the element $\gamma$ can be generated by a 1-periodic Hamiltonian which is $\geq C \cdot E(z)$. But by the definition of $\Gamma$ we have $\gamma \theta_{\text {st }}=\gamma$. The claim follows because $\theta_{\text {st }}^{N}=\theta_{s t}$ for all $N \in \mathbb{N}$ and hence $\gamma$ can be generated by a contact Hamiltonian which is $\geq C \cdot E(z)$ with an arbitrarily large $C$.

Let us apply this result to the case when $(P, \eta)$ is the standard contact sphere $S^{2 n-1}$. The following proposition shows that, despite the fact that the sphere is not orderable for $n \geq 2$, the semigroup

$$
\mathcal{R}\left(S^{2 n-1}\right)=(\vec{G} \backslash \Gamma) \cup\{0\}
$$

contains a subset of cardinality continuum. Let $\epsilon_{c} \in \vec{G}, c>0$, be the element represented by the path

$$
z \rightarrow e^{2 \pi i c t} z
$$

with $t \in[0,1]$. 
Proposition 6.13 $\epsilon_{c} \in \vec{G} \backslash \Gamma$ for each $c \in(0,1)$

Proof of Proposition 6.13 It follows from Theorem 1.2 that if $c \in(0,1)$ then the domain $U\left(\epsilon_{c}\right)=\widehat{B}^{2 n}\left(\frac{1}{c}\right)$ cannot be squeezed into $\widehat{B}^{2 n}(1)$. Then Proposition 6.12 yields $\epsilon_{c} \in \vec{G} \backslash \Gamma$. This completes the proof.

Furthermore, the partial order $\underset{+}{\succeq}$ on $\mathcal{R}$ is non-trivial since $\epsilon_{a} \succeq \epsilon_{b}$ for $1>a>b>0$.

\subsection{Quantum product in contact homology and other useful tools}

Let $(P, \eta)$ be an ideal contact boundary of a Liouville manifold $(M, \omega, L)$. Under suitable assumptions on the first Chern class of $(M, \omega)$ (see Section 4 above) we have a well defined contact homology theory for fiberwise starshaped domains in $M \times S^{1}$. Denote by $\mathrm{CH}(\gamma)$ the contact homology of the domain $U(\gamma)$. It would be interesting to relate the correspondence $\gamma \rightarrow \mathrm{CH}(\gamma)$ with the multiplication in $\vec{G}$. For instance, it sounds likely that there exists a natural pairing

$$
\mathrm{CH}\left(\gamma_{1}\right) \otimes \mathrm{CH}\left(\gamma_{2}\right) \rightarrow \mathrm{CH}\left(\gamma_{1} \gamma_{2}\right)
$$

whose construction imitates the pair of pants product in Floer homology. This pairing is a potentially useful tool for studying the semigroup $\vec{G}(P, \eta)$.

It would be interesting to elaborate this idea in the case when $(P, \eta)$ is a prequantization space of a closed symplectic manifold $(N, \sigma)$. Write $p: P \rightarrow N$ for the natural projection, denote by $e_{t}$ the natural circle action on $P$, and write $\beta$ for the connection 1-form on $P$ whose kernel equals $\eta$.

For instance, think of the Hopf fibration $S^{2 n-1} \rightarrow \mathbb{C} P^{n-1}$ as of the prequantization of the complex projective space equipped with a suitable multiple of the Fubini-Study form. Here the sphere is considered as the ideal contact boundary of $\mathbb{C}^{n}$, and $e_{t}(z)=e^{2 \pi i t} z$.

Returning to the general setting, take any path of Hamiltonian diffeomorphisms $\left\{h_{t}\right\}$ on $N$ representing an element $\tilde{h}$ in the universal cover $\operatorname{Ham} \tilde{(N}, \sigma)$ of the group of Hamiltonian diffeomorphisms of $(N, \sigma)$. We can lift it to a path of contactomorphisms of $(P, \eta)$ in the following way. Let us normalize the Hamiltonian $H_{t}$ on $N$ generating $\left\{h_{t}\right\}$ by requiring that $H_{t}$ has zero mean with respect to the symplectic volume on $N$. Look at the projection $S P \rightarrow P \rightarrow N$. Take the (time-dependent) contact Hamiltonian on $S P$ whose restriction to $\operatorname{graph}(\beta) \subset S P$ coincides with the lift of $H_{t}$. This Hamiltonian generates a flow, say $f_{t}$, which is a lift of the original Hamiltonian flow $h_{t}$. Note now that the path $\left\{e_{c t} f_{t}\right\}, t \in[0,1]$, is positive for sufficiently large $c$ and hence we get a well defined element $\gamma_{c} \in \vec{G}$. Thus we can associate to $\left.\tilde{h} \in \operatorname{Ham} \tilde{(N}, \sigma\right)$ 
a 1-parametric family of vector spaces $\mathrm{CH}\left(\gamma_{c}\right)$, where $c$ is sufficiently large. It would be interesting to establish a link between this family of vector spaces and Hamiltonian Floer homology of $\tilde{h}$.

It seems also useful to develop an equivariant version of the contact homology theory. For instance, $\mathbb{Z} / 2$-equivariant contact homology for domains in $\mathbb{R}^{2 n} \times S^{1}$ may help recovering Givental's result about the orderability of the standard contact structure on $\mathbb{R} P^{2 n-1}$.

\section{Appendix A: Proof of Lemma 4.17}

The proof is divided into several steps.

Step 1 Note that $F$ is a proper map. Denote by $\Sigma$ the image of $F$. A point $p \in \Sigma$ is regular if $\Sigma$ is a smooth submanifold of $W$ in a neighborhood of $p$, otherwise $p$ is called singular. The Micallef-White theorem [38] states that locally a non-parametrized $J$-holomorphic curve is $C^{1}$-diffeomorphic to a usual holomorphic curve. Thus singular points form a discrete subset $\operatorname{Sing}(\Sigma)$ in $\bar{V}$, and near a singular point $p$ the set $\Sigma \backslash\{p\}$ is a union of a finite number of punctured discs. This enables us to perform

normalization of $\Sigma$ (eg see Chirka [7]). We get a Riemann surface $\widetilde{\Sigma}$ together with a proper holomorphic map $\pi: \widetilde{\Sigma} \rightarrow \Sigma$ such that $\pi$ is a diffeomorphism outside a discrete subset $S \subset \widetilde{\Sigma}$ and $\pi(S)$ is exactly the set of singular points of $\Sigma$. Furthermore there exists a proper lift $\widetilde{F}: \Upsilon \rightarrow \widetilde{\Sigma}$ such that $F=\pi \circ \widetilde{F}$.

We claim that the map $\tilde{F}$ is a diffeomorphism. To see this, denote by $k$ and $g$ the number of ends and the genus of $\widetilde{\Sigma}$, respectively. Since $\gamma_{+} \neq \gamma_{-}$we have $k \geq 2$. Let $d$ be the degree of $\widetilde{F}$ and $r$ the total number of singular points counted with the multiplicites. By the Riemann-Hurwitz formula, where we use that $\Upsilon$ is a cylinder, we have

$$
d(2 g-2+k)+r=0 .
$$

Since $d \geq 1, g \geq 0, k \geq 2$ and $r \geq 0$ we obtain that $g=r=0$ and $k=2$. In particular, $\widetilde{\Sigma}$ is a cylinder and, since $r=0$, the map $\widetilde{F}$ is a non-ramified covering. Taking into account that the orbits $\gamma_{+}$and $\gamma_{-}$are simple, we conclude that $d=1$, and hence $\tilde{F}$ is a diffeomorphism. The claim is proved.

In view of this discussion we can assume that $F$ itself is a diffeomorphism of $\Upsilon \backslash$ $\operatorname{Sing}(F)$ onto $\Sigma \backslash \operatorname{Sing}(\Sigma)$. Note that the Cauchy-Riemann equation (48) implies that the vertical component $\varphi$ of a $J$-holomorphic map is related to its $V$-component $f$ by the equation

$$
d \varphi=f^{*} \lambda \circ i
$$


where $i$ is the complex structure on $\Upsilon$ and $\lambda$ is the 1 -form defining the framing of $V$.

Step 2 Let us denote by $\operatorname{Crit}(f) \subset \Upsilon$ the set of critical points of $d f$. In view of the Cauchy-Riemann equation, the image $F(\operatorname{Crit}(f))$ corresponds to the tangency points of $\Sigma$ with the $J$-holomorphic characteristic foliation of $\bar{V}$. It follows from the generalized similarity principle due to Hofer and Zehnder [32] that Crit $(f)$ is a discrete subset of $\Upsilon$. This yields that

$$
\operatorname{Closure}(f(\operatorname{Crit}(f))) \subset f(\operatorname{Crit}(f)) \cup \gamma_{-} \cup \gamma_{+} .
$$

Step 3 Outside the set Crit $(f)$ the map $f$ is non-tangent to the characteristic foliation of $V$, and in particular to the periodic orbits at infinity $\gamma_{-} \cup \gamma_{+}$. Thus the (clearly, open) set $\Upsilon^{\prime}=\Upsilon \backslash f^{-1}\left(\gamma_{-} \cup \gamma_{+}\right)$is dense in $\Upsilon$.

Step 4 We claim that the injectivity points lying in $\Upsilon^{\prime}$ form an open subset. Indeed, let $z \in \Upsilon^{\prime}$ be an injectivity point. Assume on the contrary that there exists a sequence $\left\{z_{j}\right\} \rightarrow z$ such that $z_{j}$ is not an injectivity point. Note that $d_{z_{j}} f \neq 0$ for large $j$ since $d_{z} f \neq 0$. Thus, after passing to a subsequence, we can assume that there exists another sequence $\left\{w_{j}\right\}$ such that $w_{j} \neq z_{j}$ for all $j$ and $f\left(w_{j}\right)=f\left(z_{j}\right)$. Since $f$ is injective in a small neighborhood of $z$ the sequence $\left\{w_{j}\right\}$ does not have $z$ as a limit point. If it has another limit point, say $w$, we have $f(z)=f(w)$ which contradicts to the fact that $z$ is an injectivity point. Thus $\left\{w_{j}\right\}$ contains an unbounded subsequence. Since $f(z)=\lim f\left(w_{j}\right)$ we conclude that $f(z) \in \gamma_{-} \cup \gamma_{+}$in contradiction with the definition of the set $\Upsilon^{\prime}$.

Step 5 Put

$$
\Upsilon_{0}=\Upsilon \backslash f^{-1}\left(f(\operatorname{Crit}(f)) \cup \gamma_{-} \cup \gamma_{+}\right)=\Upsilon^{\prime} \backslash f^{-1}(f(\operatorname{Crit}(f))) .
$$

This set is open in view of inclusion (88). Let us check that it is dense in $\Upsilon^{\prime}$ (and hence dense in $\Upsilon$ ). Indeed, assume on the contrary that this is not the case. Then there exists an open subset, say $P \subset \Upsilon^{\prime}$, with $f(P) \subset f(\operatorname{Crit}(f))$. But $f(\operatorname{Crit}(f))$ is a countable subset of $V$ (see Step 2) so $d f$ vanishes identically on $P$. Equation (87) yields that $d \phi=0$ on $P$ as well. Thus $d F=0$ on $P$, and by the unique continuation argument the map $F$ is constant, a contradiction.

Step 6 It suffices to show that injectivity points are dense in $\Upsilon_{0}$. Take any point $z \in \Upsilon_{0}$. There exist neighborhoods $D$ of $z$ and $U$ of $f(z)$ with the following properties.

- $f(D) \subset U$; 
- $U \cap\left(\gamma_{-} \cup \gamma_{+}\right)=\varnothing$;

- $\left.f\right|_{D}$ is an embedding.

Let as analyze injective points in $D$. Notice first that in view of the boundary conditions for $f$ there exists a sufficiently large closed annulus $A \subset \Upsilon$ containing $D$ and such that $U \cap f(\Upsilon \backslash A)=\varnothing$. Furthermore, every point in $f^{-1}(f(z))$ is non-critical for $d f$ due to our definition of $\Upsilon_{0}$. Thus, enlarging, if necessary, $A$ and shrinking $D$ and $U$, we can achieve that $f^{-1}(U) \cap A$ is a union of a finite number of pairwise disjoint open discs $B_{1}, \ldots, B_{N}$ such that $\left.f\right|_{B_{j}}$ is an embedding. One of these discs, say $B_{1}$, contains $D$. Put $B=B_{j}$ with $j \geq 2$. We claim that $D \backslash f^{-1}(f(D) \cap f(B))$ is dense in $D$. Indeed, otherwise there exist open subsets $D^{\prime} \subset D$ and $B^{\prime} \subset B$ such that $f\left(D^{\prime}\right)=f\left(B^{\prime}\right)$, and hence there is a holomorphic diffeomorphism $\psi: D^{\prime} \rightarrow B^{\prime}$ such that $\left.f\right|_{B^{\prime}} \circ \psi=\left.f\right|_{D^{\prime}}$. Equation (87) yields $\left.\varphi\right|_{B^{\prime}} \circ \psi=\left.\varphi\right|_{D^{\prime}}+c$ where $c \in \mathbb{R}$ is a constant. If $c=0$ we have $\left.F\right|_{D^{\prime}}=\left.F\right|_{B^{\prime}}$ which contradicts Step 1 of the proof. Thus $c \neq 0$. Recall that the adjusted almost complex structure $J$ on $\bar{V}=V \times \mathbb{R}$ is invariant under the shift $T_{c}:(y, s) \rightarrow(y, s+c)$ along the $\mathbb{R}$-direction. Thus our conclusion above can be reformulated as follows: the cylinder $\Sigma=F(\Upsilon)$ and $T_{c}(\Sigma)$ intersect over an open subset. By the unique continuation argument, we have that $\Sigma=T_{c}(\Sigma)$. Thus $\Sigma=T_{N c}(\Sigma)$ for all $N \in \mathbb{Z}$. Taking $N \rightarrow \pm \infty$ and looking at the boundary conditions, we get that $\gamma_{-}=\gamma_{+}$, which contradicts our assumption that the orbits at infinity are distinct.

Thus we proved that every point $z_{0} \in \Upsilon_{0}$ has a neighborhood where injective points are dense. This completes the proof.

\section{Appendix B: The Olshanskii criterion and non-orderability of $S^{3}$}

In this Appendix we prove that the cone $C$ in the Lie algebra of $P U(2,1)$ induced from the cone of non-negative contact Hamiltonians under the natural monomorphism $P U(2,1) \rightarrow \operatorname{Cont}\left(S^{3}\right)$ does not generate a genuine partial order. For the proof we apply Olshanskii's criterion [39]. The text below is based in parts on the notes which were kindly supplied to us by G Olshanskii. These notes contain a translation of this criterion, which is formulated in [39] in the abstract language of Lie theory, into the language of matrices. Below we present an exposition of Olshanskii's criterion in the context of $P U(2,1)$.

Consider the form $q(u, w)$ on $\mathbb{C}^{3}$ given by

$$
q(u, w)=u_{1} \bar{w}_{1}+u_{2} \bar{w}_{2}-u_{3} \bar{w}_{3} .
$$


By definition, $U(2,1)$ is the group of all $\mathbb{C}$-linear transformations preserving $q$. In particular, this group preserves the set

$$
\left\{u \in \mathbb{C}^{3}: q(u, \bar{u})<0\right\} .
$$

Passing to the projectivization and setting $z_{1}=u_{1} / u_{3}$ and $z_{2}=u_{2} / u_{3}$, we get that the group $P U(2,1):=U(2,1) / S^{1}$ acts by biholomorphic automorphisms of the ball

$$
B^{4}:=\left\{z \in C^{2}:\left|z_{1}\right|^{2}+\left|z_{2}\right|^{2}<1\right\} .
$$

These automorphisms extend to diffeomorphisms of the boundary sphere $S^{3}$. They obviously preserve the field of complex tangent lines to $S^{3}$, and, therefore, act by contactomorphisms of the standard contact $S^{3}$.

We identify the real Lie algebra $\mathfrak{g}$ of $P U(2,1)$ with $\operatorname{su}(2,1)$. It will be useful to describe $\mathfrak{g}$ explicitly. It consists of those traceless complex matrices $A$ of the order $3 \times 3$ which satisfy $A^{*} I+I A=0$, where

$$
I=\left(\begin{array}{ccc}
1 & 0 & 0 \\
0 & 1 & 0 \\
0 & 0 & -1
\end{array}\right),
$$

and $A^{*}$ stands for the complex conjugate of the transposed matrix to $A$. Introduce the following basis in $\mathfrak{g}$ :

$$
\begin{aligned}
E_{1} & =\left(\begin{array}{ccc}
i & 0 & 0 \\
0 & 0 & 0 \\
0 & 0 & -i
\end{array}\right), E_{2}=\left(\begin{array}{ccc}
0 & 0 & 0 \\
0 & i & 0 \\
0 & 0 & -i
\end{array}\right), \\
F & =\left(\begin{array}{ccc}
0 & 1 & 0 \\
-1 & 0 & 0 \\
0 & 0 & 0
\end{array}\right), \widetilde{F}=\left(\begin{array}{ccc}
0 & i & 0 \\
i & 0 & 0 \\
0 & 0 & 0
\end{array}\right), \\
G_{1} & =\left(\begin{array}{lll}
0 & 0 & 1 \\
0 & 0 & 0 \\
1 & 0 & 0
\end{array}\right), \widetilde{G}_{1}=\left(\begin{array}{ccc}
0 & 0 & i \\
0 & 0 & 0 \\
-i & 0 & 0
\end{array}\right), \\
G_{2} & =\left(\begin{array}{lll}
0 & 0 & 0 \\
0 & 0 & 1 \\
0 & 1 & 0
\end{array}\right), \widetilde{G}_{2}=\left(\begin{array}{ccc}
0 & 0 & 0 \\
0 & 0 & i \\
0 & -i & 0
\end{array}\right) .
\end{aligned}
$$

Consider a Cartan subalgebra $\mathfrak{h}=\operatorname{Span}_{\mathbb{R}}\left(E_{1}, E_{2}\right)$. Our next task is to describe explicitly the cone $C \cap \mathfrak{h}$. Let us introduce symplectic polar coordinates

$$
\rho_{k}=\pi\left|z_{k}\right|^{2}, \varphi_{k}=\frac{1}{2 \pi} \operatorname{Arg}\left(z_{k}\right), k=1,2,
$$


in $\mathbb{C}^{2}$. With this notation the standard contact form on $S^{3}$ can be written as

$$
\beta=\rho_{1} d \varphi_{1}+\rho_{2} d \varphi_{2} .
$$

Note that the element $E=a E_{1}+b E_{2} \in \mathfrak{h}$ generates the flow

$$
u \mapsto\left(\begin{array}{ccc}
e^{i a t} & 0 & 0 \\
0 & e^{i b t} & 0 \\
0 & 0 & e^{-i(a+b) t}
\end{array}\right) u
$$

on $\mathbb{C}^{3}$ and, therefore, the flow

$$
z \mapsto\left(\begin{array}{cc}
e^{i(2 a+b) t} & 0 \\
0 & e^{i(a+2 b) t}
\end{array}\right) z
$$

on $\mathbb{C}^{2}$. When restricted to $S^{3}$, the latter flow is generated by the vector field

$$
X=\frac{1}{2 \pi}(2 a+b) \frac{\partial}{\partial \varphi_{1}}+\frac{1}{2 \pi}(a+2 b) \frac{\partial}{\partial \varphi_{2}} .
$$

By definition, $E \in C$ if and only if $\beta(X) \geq 0$ everywhere on $S^{3}$, which means that both coefficients $2 a+b$ and $2 b+a$ are non-negative. Summing up, we get

$$
C \cap \mathfrak{h}=\{(a, b): 2 a+b \geq 0, a+2 b \geq 0\},
$$

where $(a, b)$ are coordinates which correspond to the basis $E_{1}, E_{2}$ of $\mathfrak{h}$.

Denote by $\mathfrak{g}_{\mathbb{C}}$ the complexified Lie algebra $\mathfrak{g} \otimes \mathbb{C}$. In order to avoid the conflict of notation, we denote by $j$ the natural complex structure on $\mathfrak{g}_{\mathbb{C}}$, that is we set $j x:=x \otimes i$ Following the notation from [39], we put

$$
\mathfrak{h}_{R e}=j \mathfrak{h} \subset \mathfrak{g}_{\mathbb{C}} .
$$

Theorem 8.1 [39] There exists a cone $c_{0} \subset \mathfrak{h}_{R e}$ with the following property: Given any convex cone $K \subset \mathfrak{g}$ which is invariant under the adjoint action, it defines a genuine partial order on the universal cover of the Lie group if and only if

$$
j K \cap \mathfrak{h}_{\operatorname{Re}} \subset \pm c_{0} .
$$

In view of (89) we have to apply this theorem to the cone

$$
c=\{(a, b): 2 a+b \geq 0, a+2 b \geq 0\},
$$

where the $(a, b)$ coordinates on $\mathfrak{h}_{R e}$ correspond to the basis $j E_{1}, j E_{2}$.

Next, we present Olshanskii's algorithm which enables one to describe explicitly the cone $c_{0}$ appearing in Theorem 8.1 in terms of the structure theory of the Lie algebra $\mathfrak{g}$. 


\section{Structure theory of $s u(2,1)$}

We start with the explicit form of operators $a d\left(E_{1}\right)$ and $a d\left(E_{2}\right)$. In order to describe these operators it will be convenient to introduce the matrix

$$
J=\left(\begin{array}{cc}
0 & -1 \\
1 & 0
\end{array}\right)
$$

and to consider the direct sum decomposition

$$
\mathfrak{g}=\operatorname{Span}_{\mathbb{R}}\left(E_{1}, E_{2}\right) \oplus \operatorname{Span}_{\mathbb{R}}(F, \widetilde{F}) \oplus \operatorname{Span}_{\mathbb{R}}\left(G_{1}, \widetilde{G}_{1}\right) \oplus \operatorname{Span}_{\mathbb{R}}\left(G_{2}, \widetilde{G}_{2}\right) .
$$

One readily calculates that $\operatorname{ad}\left(E_{1}\right)$ is given by the matrix

$$
0 \oplus J \oplus 2 J \oplus J
$$

and $\operatorname{ad}\left(E_{2}\right)$ is given by the matrix

$$
0 \oplus(-J) \oplus J \oplus 2 J
$$

Up to a positive multiple, the Killing form $Q$ restricted to $\mathfrak{h}_{R e}$ is given by the matrix

$$
\left(\begin{array}{ll}
2 & 1 \\
1 & 2
\end{array}\right)
$$

in the basis $\left(j E_{1}, j E_{2}\right)$. The space $\mathfrak{h}_{R e}$ can be identified with the dual to $\mathfrak{h}$ with the help of the Killing form. Hence, we can assume that the roots of $\left(\mathfrak{g}_{\mathbb{C}}, \mathfrak{h}_{\mathbb{C}}\right)$ lie in $\mathfrak{h}_{R e}$. Diagonalizing operators $a d\left(E_{1}\right)$ and $\operatorname{ad}\left(E_{2}\right)$ we calculate that in the above basis the roots have the form

$$
\pm \gamma= \pm(1,-1), \pm \alpha_{1}= \pm(1,0), \pm \alpha_{2}= \pm(0,1) .
$$

The root system is of the type $A_{2}$, and one can declare $\gamma, \alpha_{1}, \alpha_{2}$ to be positive roots.

Next, we have the Cartan decomposition $\mathfrak{g}=\mathfrak{t} \oplus \mathfrak{p}$ into the direct sum of the subspaces formed by skew-Hermitian and Hermitian matrices respectively. Thus $\mathfrak{t}=$ $\operatorname{Span}_{\mathbb{R}}\left(E_{1}, E_{2}, F, \widetilde{F}\right)$ and $\mathfrak{p}=\operatorname{Span}_{\mathbb{R}}\left(G_{1}, \widetilde{G}_{1}, G_{2}, \widetilde{G}_{2}\right)$. Recall that a root is called non-compact if the corresponding eigenspace is contained in $\mathfrak{p} \otimes \mathbb{C}$. It is easy to verify that the positive non-compact roots are given by $\alpha_{1}$ and $\alpha_{2}$. Denote by $c_{m i n}$ the cone in $\mathfrak{h}_{R e}$ generated by these roots:

$$
c_{\min }=\{(a, b): a \geq 0, b \geq 0\},
$$

where the $(a, b)$-coordinates on $\mathfrak{h}_{R e}$ correspond to the basis $j E_{1}, j E_{2}$.

Note that the center $\zeta$ of the algebra $t$ is generated by the vector $E_{1}+E_{2}$. Furthermore, the roots of the algebra $\mathfrak{t}_{\mathbb{C}}$ with respect to the Cartan subalgebra $\mathfrak{h}_{\mathbb{C}}$ are given by $\pm \gamma$. 
Let $W$ be the corresponding Weyl group which acts on $\mathfrak{h}_{R e}$ by the permutation of coordinates in the basis $\left(j E_{1}, j E_{2}\right)$.

\section{Construction of the cone $c_{0}$}

We work with the basis $\left(j E_{1}, j E_{2}\right)$ of $\mathfrak{h}_{R e}$.

Step 1 Choose a maximal subsystem of positive non-compact pairwise orthogonal roots. Since $\alpha_{1}$ and $\alpha_{2}$ form the angle $\pi / 3$, we choose only one of them, say $\alpha_{1}$. Denote by $H_{1}$ the vector of the form $s \alpha_{1}$ with $s>0$ so that

$$
Q\left(\alpha_{1}, H_{1}\right)=2 \text {. }
$$

We calculate that $H_{1}=(1,0)$.

Step 2 Choose the vector $Z \in j \zeta$ such that $Q\left(\alpha_{1}, Z\right)=Q\left(\alpha_{2}, Z\right)=2$. We calculate that $Z=(2 / 3,2 / 3)$. Put

$$
H_{0}=Z-H_{1}=\frac{1}{3} \cdot(-1,2)
$$

Step 3 Consider the cone $c_{1}$ spanned by $W H_{0} \cup c_{\min }$. Note that $c_{1}$ is spanned by vectors

$$
\frac{1}{3} \cdot(-1,2) \text { and } \frac{1}{3} \cdot(2,-1) \text {. }
$$

The cone $c_{0}$ from Theorem 8.1 is defined as the cone whose dual with respect to the form $Q$ equals $c_{1}$ :

$$
c_{1}=\left\{x \in \mathfrak{h}_{R e}: Q(x, y) \geq 0 \forall y \in c_{0}\right\} .
$$

One readily calculates that $c_{0}=c_{m i n}$, where $c_{\text {min }}$ is given by inequalities (91).

The final step Recalling that the cone $c=j C \cap \mathfrak{h}_{R e}$ is given by inequalities (90) we get that $c_{0}$ is strictly contained in $c$. Thus, by Theorem 8.1 the cone $C$ does not generate a genuine partial order.

Remark 8.2 Olshanskii's method does not give rise to an explicit description of a positive contractible loop of contactomorphisms which lies in $P U(2,1) \subset \operatorname{Cont}\left(S^{3}\right)$. However, imitating our construction in Section 3.5 above, we present here such a loop. Let us identify $P U(2,1)$ with the group of complex automorphisms of $B^{4}$. We define a distinguished contactomorphism (cf Section 3.4 above) by the formula

$$
b\left(z_{1}, z_{2}\right)=\left(\frac{\cosh \alpha \cdot z_{1}+1}{z_{1}+\cosh \alpha}, \frac{\sinh \alpha \cdot z_{2}}{z_{1}+\cosh \alpha}\right) .
$$


Put $e_{t} z:=e^{2 \pi i t} z$ and

$$
f_{t}\left(z_{1}, z_{2}\right)=\left(e^{2 \pi i t} z_{1}, e^{-2 \pi i t} z_{2}\right),
$$

as in Section 3.5 above. A lengthy but straightforward calculation shows that the (obviously contractible) loop $e_{-t} f_{3 t} b e_{t} b^{-1}$ (cf Theorem 3.6) is positive, provided that $\alpha>0$ is small enough.

In order to reveal the geometry of the distinguished contactomorphism $b$, let us perform the Cayley transform [42, Chapter 2.3]

$$
\Phi: B^{4} \rightarrow \Omega, \quad\left(z_{1}, z_{2}\right) \mapsto \frac{i}{1-z_{1}}\left(z_{1}+1, z_{2}\right),
$$

where

$$
\Omega=\left\{\left(w_{1}, w_{2}\right) \in \mathbb{C}^{2}: \operatorname{Im} w_{1}>\left|w_{2}\right|^{2}\right\}
$$

is a multi-dimensional analogue of the upper half-plane. This transform is a biholomorphism between Closure $\left(B^{4}\right) \backslash\{(1,0)\}$ and Closure $(\Omega)$. Let $\eta$ be the field of complex tangent lines to $\partial \Omega$. Put $w_{1}=x+i y, w_{2}=u+i v$. One readily shows that in the coordinates $(x, u, v)$ the contact manifold $(\partial \Omega, \eta)$ is simply $\mathbb{R}^{3}$ equipped with the standard contact structure

$$
\operatorname{Ker}(d x+2(u d v-v d u)) .
$$

The map $\hat{b}:=\Phi b \Phi^{-1}: \Omega \rightarrow \Omega$ turns out to be a non-isotropic dilation

$$
\left(w_{1}, w_{2}\right) \mapsto\left(s^{2} w_{1}, s w_{2}\right),
$$

where $s \rightarrow+\infty$ as $\alpha \rightarrow 0$. Of course, the restriction of $\hat{b}$ to the boundary has the friendly form

$$
(x, u, v) \rightarrow\left(s^{2} x, s u, s v\right)
$$

Remark 8.3 It is unclear to us whether the explicit analytic formula for the positive contractible loop in $P U(2,1) \subset \operatorname{Cont}\left(S^{3}\right)$ presented above helps to simplify our calculations in Section 3, namely to "extend" this loop to a nonnegative contractible loop in stabilizations, and to calculate the sharp lower bound for the invariant $\mu(\Delta)$ defined by formula (1).

Remark 8.4 Oshanskii's paper [39] provides information on the orderability of very general finite-dimensional simply-connected Lie groups in terms of the structure theory. This subject was further developed in $[29 ; 30]$. It would be interesting to explore the notions introduced in Section 6.3 in this context. 


\section{Acknowledgements}

We cordially thank Ms M Hercberg for preparing LaTex figures for this paper. We are indebted to E Giroux for pointing out that our Theorem 1.10 follows from the Olshanskii criterion. We are grateful to $\mathrm{G}$ Olshanskii for providing illuminating notes which helped us a lot to understand his paper [39] and for useful comments on the first draft of Appendix B. We thank J McCleary for providing us with reference [20], R Cohen, S Goberstein, M Sapir and S Weinberger for useful consultations, D McDuff, F Schlenk and the anonymous referee for useful comments on the manuscript.

Our numerous meetings at Stanford University, American Institute of Mathematics (Palo Alto), Institute of Advanced Studies (Princeton) and Tel Aviv University were indispensable for completing this project. We express our gratitude to these institutions. The third named author is grateful to the Center for Dynamics and Geometry at Pennstate for the opportunity to lecture on preliminary results of the present paper in Spring 2005, and in particular to D Burago and S Tabachnikov for their warm hospitality.

In its early stages, this project was supported in part by United States-Israel Binational Science Foundation grant number 1999086.

The authors acknowledge the support of the following grants:

Y Eliashberg NSF grants DMS-0204603 and DMS-0244663

S S Kim NSF grant DMS-972992, WISE fellowship from the University of Southern California and FCT/SFRH/BPD (Portugal)

L Polterovich Michael Bruno Memorial Award

\section{References}

[1] A Abbondandolo, M Schwarz, On the Floer homology of cotangent bundles, Comm. Pure Appl. Math. 59 (2006) 254-316 MR2190223

[2] G Baumslag, A G Myasnikov, V Shpilrain, Open problems in combinatorial group theory. Second edition, from: "Combinatorial and geometric group theory (New York, 2000/Hoboken, NJ, 2001)”, Contemp. Math. 296, Amer. Math. Soc., Providence, RI (2002) 1-38 MR1921705

[3] P Biran, L Polterovich, D Salamon, Propagation in Hamiltonian dynamics and relative symplectic homology, Duke Math. J. 119 (2003) 65-118 MR1991647

[4] F Bourgeois, A Morse-Bott approach to contact homology, from: "Symplectic and contact topology: interactions and perspectives (Toronto, ON/Montreal, QC, 2001)", (Y Elashberg, B Khesin, F Lalonde, editors), Fields Inst. Commun. 35, Amer. Math. Soc., Providence, RI (2003) 55-77 MR1969267 
[5] F Bourgeois, Contact homology and homotopy groups of the space of contact structures, Math. Res. Lett. 13 (2006) 71-85 MR2200047

[6] F Bourgeois, Y Eliashberg, H Hofer, K Wysocki, E Zehnder, Compactness results in symplectic field theory, Geom. Topol. 7 (2003) 799-888 MR2026549

[7] E M Chirka, Complex analytic sets, Mathematics and its Applications (Soviet Series) 46, Kluwer Academic Publishers Group, Dordrecht (1989) MR1111477 Translated from the Russian by R A M Hoksbergen

[8] K Cieliebak, Subcritical manifolds are split arXiv:math.DG/0204351

[9] K Cieliebak, A Floer, H Hofer, Symplectic homology. II. A general construction, Math. Z. 218 (1995) 103-122 MR1312580

[10] K Cieliebak, V L Ginzburg, E Kerman, Symplectic homology and periodic orbits near symplectic submanifolds, Comment. Math. Helv. 79 (2004) 554-581 MR2081726

[11] K Cieliebak, H Hofer, J Latschev, F Schlenk, Quantitative symplectic geometry arXiv:math.SG/0506191

[12] M de Gosson, The symplectic camel and phase space quantization, J. Phys. A 34 (2001) 10085-10096 MR1872403

[13] M de Gosson, Phase space quantization and the uncertainty principle, Phys. Lett. A 317 (2003) 365-369 MR2027474

[14] D L Dragnev, Fredholm theory and transversality for noncompact pseudoholomorphic maps in symplectizations, Comm. Pure Appl. Math. 57 (2004) 726-763 MR2038115

[15] Y Eliashberg, Topological characterization of Stein manifolds of dimension > 2, Internat. J. Math. 1 (1990) 29-46 MR1044658

[16] Y Eliashberg, New invariants of open symplectic and contact manifolds, J. Amer. Math. Soc. 4 (1991) 513-520 MR1102580

[17] Y Eliashberg, A Givental, H Hofer, Introduction to symplectic field theory, GAFA 2000 (Tel Aviv, 1999), Geom. Funct. Anal. (2000) 560-673 MR1826267

[18] Y Eliashberg, M Gromov, Convex symplectic manifolds, from: "Several complex variables and complex geometry, Part 2 (Santa Cruz, CA, 1989)", Proc. Sympos. Pure Math. 52, Amer. Math. Soc., Providence, RI (1991) 135-162 MR1128541

[19] Y Eliashberg, L Polterovich, Partially ordered groups and geometry of contact transformations, Geom. Funct. Anal. 10 (2000) 1448-1476 MR1810748

[20] Y Félix, J C Thomas, M Vigué-Poirrier, Free loop spaces of finite complexes have infinite category, Proc. Amer. Math. Soc. 111 (1991) 869-875 MR1025277

[21] A Floer, Symplectic fixed points and holomorphic spheres, Comm. Math. Phys. 120 (1989) 575-611 MR987770

[22] A Floer, H Hofer, Symplectic homology. I. Open sets in $\mathbf{C}^{n}$, Math. Z. 215 (1994) 37-88 MR1254813 
[23] A Floer, H Hofer, K Wysocki, Applications of symplectic homology. I, Math. Z. 217 (1994) 577-606 MR1306027

[24] H Geiges, Contact geometry, from: "Handbook of differential geometry. Vol. II", Elsevier/North-Holland, Amsterdam (2006) 315-382 MR2194671

[25] V L Ginzburg, B Z Gürel, Relative Hofer-Zehnder capacity and periodic orbits in twisted cotangent bundles, Duke Math. J. 123 (2004) 1-47 MR2060021

[26] E Giroux, Convexité en topologie de contact, Comment. Math. Helv. 66 (1991) 637-677 MR1129802

[27] A Givental, Nonlinear generalization of the Maslov index, from: "Theory of singularities and its applications", Adv. Soviet Math. 1, Amer. Math. Soc., Providence, RI (1990) 71-103 MR1089671

[28] M Gromov, Pseudoholomorphic curves in symplectic manifolds, Invent. Math. 82 (1985) 307-347 MR809718

[29] J Hilgert, K-H Neeb, Lie semigroups and their applications, Lecture Notes in Mathematics 1552, Springer, Berlin (1993) MR1317811

[30] J Hilgert, G Ólafsson, Causal symmetric spaces, Geometry and harmonic analysis, Perspectives in Mathematics 18, Academic Press, San Diego, CA (1997) MR1407033

[31] H Hofer, K Wysocki, E Zehnder, Properties of pseudoholomorphic curves in symplectizations. III. Fredholm theory, from: "Topics in nonlinear analysis", Progr. Nonlinear Differential Equations Appl. 35, Birkhäuser, Basel (1999) 381-475 MR1725579

[32] H Hofer, E Zehnder, Symplectic invariants and Hamiltonian dynamics, Birkhäuser Advanced Texts, Birkhäuser, Basel (1994) MR1306732

[33] S Jackowski, J Słomińska, $G$-functors, $G$-posets and homotopy decompositions of $G$-spaces, Fund. Math. 169 (2001) 249-287 MR1852128

[34] F Lalonde, D McDuff, The geometry of symplectic energy, Ann. of Math. (2) 141 (1995) 349-371 MR1324138

[35] F Lalonde, D McDuff, Hofer's $L^{\infty}$-geometry: energy and stability of Hamiltonian flows. I, II, Invent. Math. 122 (1995) 1-33, 35-69 MR1354953

[36] J A Makowsky, On some conjectures connected with complete sentences, from: "Collection of articles dedicated to Andrzej Mostowski on the occasion of his sixtieth birthday, III", Fund. Math. 81 (1974) 193-202 MR0366647

[37] D McDuff, D Salamon, $J$-holomorphic curves and symplectic topology, American Mathematical Society Colloquium Publications 52, American Mathematical Society, Providence, RI (2004) MR2045629

[38] MJ Micallef, B White, The structure of branch points in minimal surfaces and in pseudoholomorphic curves, Ann. of Math. (2) 141 (1995) 35-85 MR1314031 
[39] G I Olshanskii, Invariant orderings in simple Lie groups. Solution of a problem of E. B. Vinberg, Funktsional. Anal. i Prilozhen. 16 (1982) 80-81 (Russian) MR684139 English translation: Functional Anal. Appl. 16 (1982) 311-313

[40] J Palis, Jr, W de Melo, Geometric theory of dynamical systems, Springer, New York (1982) MR669541An introduction, Translated from the Portuguese by A K Manning

[41] J Robbin, D Salamon, The Maslov index for paths, Topology 32 (1993) 827-844 MR1241874

[42] W Rudin, Function theory in the unit ball of $\mathbf{C}^{n}$, Grundlehren series 241, Springer, New York (1980) MR601594

[43] D Salamon, J Weber, Floer homology and the heat flow, Geom. Funct. Anal. (GAFA). to appear arXiv:math.SG/0304383

[44] I Ustilovsky, Infinitely many contact structures on $S^{4 m+1}$, Internat. Math. Res. Notices (1999) 781-791 MR1704176

[45] C Viterbo, Functors and computations in Floer homology with applications, Part II, preprint (1996)

[46] C Viterbo, Metric and isoperimetric problems in symplectic geometry, J. Amer. Math. Soc. 13 (2000) 411-431 MR1750956

[47] J Weber, Noncontractible periodic orbits in cotangent bundles and Floer homology, Duke Math. J. 133 (2006) 527-568 MR2228462

[48] M-L Yau, Cylindrical contact homology of subcritical Stein-fillable contact manifolds, Geom. Topol. 8 (2004) 1243-1280 MR2087083

Department of Mathematics, Stanford University

Stanford, CA 94305-2125, USA

Departamento de Matemática, Instituto Superior Técnico

Av Roviso Pais, 1049-001 Lisboa, Portugal

School of Mathematical Sciences, The Raymond and Beverly Sackler Faculty of Exact

Sciences, Tel Aviv University, 69978 Tel Aviv, Israel

eliash@math.stanford.edu, sskim@math.ist.utl.pt,

polterov@math.tau.ac.il

Proposed: Eleny Ionel

Seconded: Tomasz Mrowka, Peter Ozsváth

Received: 12 February 2006

Revised: 30 September 2006 